\title{
Advances in Optics and Photonics
}

\section{Integrated microwave photonic filters}

\author{
YANG LiU, ${ }^{1,2,5}$ (1) Amol Choudhary, ${ }^{3}$ (1) \\ David Marpaung, ${ }^{4}{ }^{\circ}$ and Benjamin J. Eggleton ${ }^{1,2,6}$
}

${ }^{1}$ Institute of Photonics and Optical Science (IPOS), School of Physics, The University of Sydney, NSW 2006, Australia

${ }^{2}$ The University of Sydney Nano Institute (Sydney Nano), The University of Sydney, NSW 2006, Australia

${ }^{3}$ Ultra Fast Optical Communications and High-performance Integrated Photonics (UFO-CHIP),

Department of Electrical Engineering, Indian Institute of Technology (IIT) Delhi, Hauz Khas,

New Delhi 110016, India

${ }^{4}$ Faculty of Science and Technology, University of Twente, 7522 NB Enschede, The Netherlands

${ }^{5}$ e-mail: yang.liu@sydney.edu.au

${ }^{6}$ e-mail: benjamin.eggleton@sydney.edu.au

Received September 23, 2019; revised February 11, 2020; accepted February 16, 2020;

published June 2, 2020 (Doc. ID 378686)

Microwave signal filtering is a fundamental and central functionality in radio-frequency (RF) systems. Underpinned by advanced integrated photonics technologies, emerging integrated microwave photonic (IMWP) filter platforms enable reconfigurable and widely tunable RF signal filtering functionalities that were unattainable using conventional electronics while also exhibiting superior features in terms of compactness, light weight, stability, low power consumption, and low latency. This paper presents a comprehensive review of the principles, architectures, and performance of IMWP filters. We highlight recent advances of IMWP filters enabled by on-chip nonlinear optics, RF-interference technology and emerging integration platforms, with an emphasis on the RF performance which is critical for their usability in real-world applications. We conclude with a perspective on future research challenges and new possibilities for IMWP filters. (c) 2020 Optical Society of America

https://doi.org/10.1364/AOP.378686

1. Introduction . . . . . . . . . . . . . . . . . . . . 487

2. IMWP Filter Architectures and Functionalities . . . . . . . . . . . . . . 491

2.1. Typical Signal Modulation Schemes for IMWP Filters . . . . . . . 492

2.2. Multi-Tap Filters . . . . . . . . . . . . . . . . . . . . . . . 494

2.3. Coherent Filter . . . . . . . . . . . . . . . . . . . . . 497

2.4. RF-Interference Filter . . . . . . . . . . . . . . . . . . 500

2.4a. Asymmetric-Dual-Sideband-Based Processing . . . . . . . . 500

2.4b. Symmetric-Dual-Sideband-Based Processing . . . . . . . 503

3. Integrated Photonic Devices and Emerging Platforms for IMWP Filters . . . 508

3.1. Photonic Devices based on Linear Optical Effects . . . . . . . . . . 509

3.1a. On-Chip Ring Resonators . . . . . . . . . . . . . . . . . 509 
3.1b. Subwavelength Periodic Structures . . . . . . . . . . . . . 513

3.1c. Ring-Assisted Mach-Zehnder Interferometer . . . . . . . . . . 514

3.2. Photonic Devices Based on Nonlinear Optical Effects . . . . . . . . . 515

3.2a. On-Chip Stimulated Brillouin Scattering . . . . . . . . . 516

3.2b. Chip-Based Optical Kerr Frequency Comb . . . . . . . . . . . 519

3.3. State-of-the-Art Advancements for IMWP Filters . . . . . . . . . . . 522

3.3a. Monolithic Integrated Microwave Photonic Filter . . . . . . 522

3.3b. Programmable and Multifunctional MWP Circuits . . . . . . . 524

3.3c. Functionality Features of State-of-the-Art Chip-Based MWP

Filters . . . . . . . . . . . . . . 526

4. IMWP Filter RF Performance . . . . . . . . . . . . . . . . . . 527

4.1. Key RF Performance Metrics . . . . . . . . . . . . . . . . . . . . . . 528

4.1a. RF Link Gain of Filter Passbands . . . . . . . . . . . . 528

4.1b. Noise Figure . . . . . . . . . . . . . . . . . . 528

4.1c. Spurious-Free Dynamic Range . . . . . . . . . . . . . . . 530

4.2. Challenges and Recent Progress in High-Performance IMWP Filters . 531

5. Conclusion . . . . . . . . . . . . . . . 536

Funding . . . . . . . . . . . . . . . . . . . 5 537

References . . . . . . . . . . . . . . . . . . 5 537 


\title{
Integrated microwave photonic filters
}

\author{
Yang Liu, Amol Choudhary, David Marpaung, \\ AND BENJAMIN J. EgGLETON
}

\section{INTRODUCTION}

Microwave filters are a basic and important building block in the front end of radiofrequency $(\mathrm{RF})$ and microwave receivers, providing signal filtering functionality to separate signals of interest from the noise background and to mitigate unwanted interference to avoid RF amplifier saturation [1,2]. Microwave photonics (MWP) [3-5] is an attractive technology for demanding filtering applications that require large instantaneous bandwidth, ultra-wideband frequency tuning, and low loss; these demanding requirements pose severe limitations to traditional RF and microwave counterparts [6]. The photonic advantages are enabled by the RF-to-optical frequency upconversion and flexible filtering in the optical domain, typically achieved using fiber-based devices, as introduced in the review article [6].

Over the recent decade, the convergence of MWP and photonic integrated circuit (PIC) [7-9] technologies has enabled the rapid development of integrated microwave photonics (IMWP). Underpinned by advanced PIC technologies, IMWP filters have been implemented by incorporating key optical components of MWP filters onto a chip-scale platform. Compared to MWP filters using bulky fiber-based optical filters, IMWP filters using centimeter-scale photonic chips exhibit dramatically reduced size, weight, and power consumption as well as enhanced stability, which are critical requirements for modern wireless communications and avionic applications [10-12]. IMWP filters also preserve the photonics-enabled advantages such as ultra-wideband frequency tunability, resulting from the ultrawide fractional frequency range of the optical frequencies. Such wideband tuning capability overcomes the existing limitation of narrow frequency tunability in state-of-the-art electronic filters [13-28]. Although wideband-tunable RF filters operating around $30 \mathrm{GHz}$ have been reported, the realization of $5 \mathrm{GHz}$ frequency tuning requires a very high control voltage exceeding $140 \mathrm{~V}$ [19], which limits their practical applications. Attractively, the tightly confined optical fields in the integrated circuits can induce strong nonlinear optical effects, producing advanced and unique signal processing and filtering functionalities that were previously unachievable for traditional microwave devices.

Figure 1(a) shows the conceptual configuration of a compact microwave system consisting of an RF antenna and an IMWP filter prior to an electronic postprocessing unit. The IMWP filter is able to perform analog signal filtering of high-frequency RF signals, without converting the RF signals to baseband frequencies through multistage RF frequency mixing and filtering. As shown in the Fig. 1 inset (i), the schematic of received signals consists of a signal of interest and a strong unwanted interferer. The IMWP filter is able to generate an RF filter response to suppress the strong interference signal while sustaining the signal of interest, as shown in the Fig. 1 inset (ii). Figure 1(b) presents a basic architecture of an IMWP filter composed of an electrooptic (E-O) modulator for RF-to-optical frequency upconversion, an integrated photonic processor for signal filtering in the optical domain, and a photodetector for optical-to-electric (O-E) frequency downconversion. To date, most efforts in IMWP filters have been made to implement key optical filtering components in compact 
photonic circuits, enabling enhanced filtering functionalities and a reduced system footprint. For the future development, the ultimate goal of IMWP filters is achieving fully integrated filter systems that incorporate light sources, modulators, photonic filtering circuits and photodetectors in one photonic chip, leading to significantly enhanced compactness and system stability, as schematically shown in Fig. 1(c).

Key milestones in the development of IMWP filters are shown in Fig. 2. The early exploration of IMWP functionalities leveraged the development of the PIC technology, starting from the advent of integrated silicon photonics [45,46] in 1985 and indium phosphide (InP) photonics [47] in 1990. The very early demonstrations of IMWP were carried out using multi-tap architectures for phased-array antennas and RF spectrum filtering in a silicon chip in 1997 [29]. In 2009, the first IMWP filter using integrated low-loss silicon resonators was reported [30], followed by the demonstration using silicon subwavelength gratings [31]. Recent rapid development of IMWP technique has benefitted from the massive progress in PICs technology, which has been driven by the investment and advancements in other fields such as data centers and telecommunications. Pioneering demonstrations of IMWP filters based on active InP photonic circuits, using cascaded ring networks [48] and cascaded Mach-Zehnder interferometers (MZIs) [32] were reported in 2010 and 2011, respectively. In the meantime, $\mathrm{Si}_{3} \mathrm{~N}_{4}$ ring resonators were demonstrated as an advantageous candidate for IMWP filters due to the ultralow optical losses [49-51]. In 2012, an ultracompact transverse IMWP filter, for the first time, was achieved using an InGaP photonic crystal waveguide that provides frequency-dependent time delay for each

\section{Figure 1}

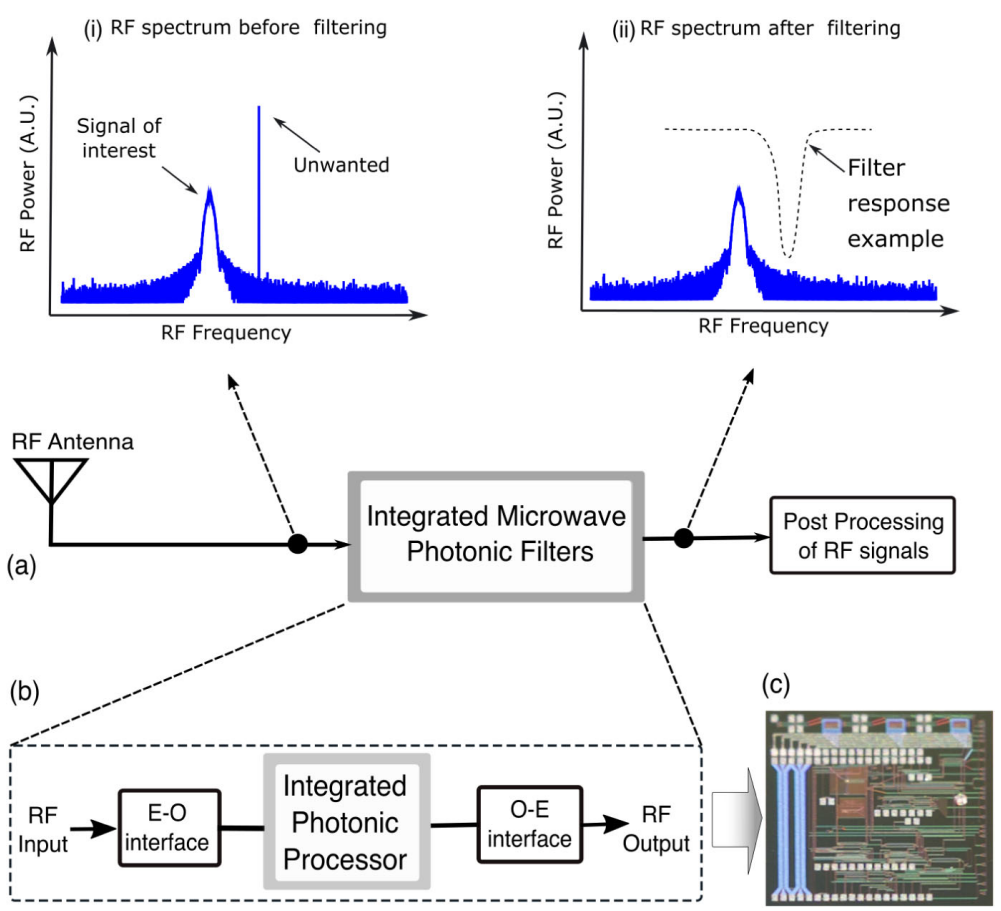

Conceptual configurations of (a) an RF receiver consisting of an RF antenna, an IMWP filter, and RF postprocessing circuits; (b) an envisaged typical IMWP filter architecture; and (c) an envisaged fully integrated IMWP filter. The inset (i) shows the schematic of RF spectrum of received RF signals consisting of a weak signal of interest and a strong unwanted interference. The inset (ii) shows the filtered RF spectrum, in which a filter response (the dashed curve) suppresses the strong interfere without attenuating the signal of interest. 
tap [33]. Recently, the emerging concept of programmable photonic circuits was extensively studied in $\mathrm{Si}_{3} \mathrm{~N}_{4}$ circuits [38] in 2015, active InP photonic circuits [39] in 2016, and in silicon circuits [42] in 2017, respectively, enabling multiple IMWP filtering functionalities using the same photonic circuit. A recent breakthrough has been made to implement a fully integrated MWP filter, achieving for the first time the $100 \%$ degree of photonic integration of an IMWP filter [40].

On-chip nonlinear optical effects, as an active optical process, started to gain great interest for implementing IMWP filters in 2012, when on-chip stimulated Brillouin scattering (SBS), induced by strong photon-phonon interactions, was harnessed as the basis of a tunable single-bandpass RF filter with a record-narrow spectral resolution of $30 \mathrm{MHz}$ [34]. An IMWP notch filter with ultrahigh selectivity was subsequently achieved using on-chip SBS with significantly reduced optical pump power [37]. On-chip optical frequency combs via the optical Kerr effect were introduced as an unprecedentedly compact laser array, providing numerous delay taps for delay-linebased IMWP filters [35] in 2014 and the Hilbert filter [36] in 2015, respectively. Such types of on-chip comb-based IMWP filters were also implemented in Hydex photonic circuits, enabling 80 delay taps to significantly improve the filter resolution $[44,52,53]$. A novel class of IMWP filter based on the simultaneous use of on-chip linear and nonlinear optical effects was recently demonstrated, achieving the synergy of optimal filter functionalities and high RF performance [43,54].

For signal filtering functionality, the spectral resolution of IMWP filters is required to be fine enough to separate the RF signals that locate in adjacent channels with a frequency separation down to tens of megahertz (MHz). However, integrated photonic

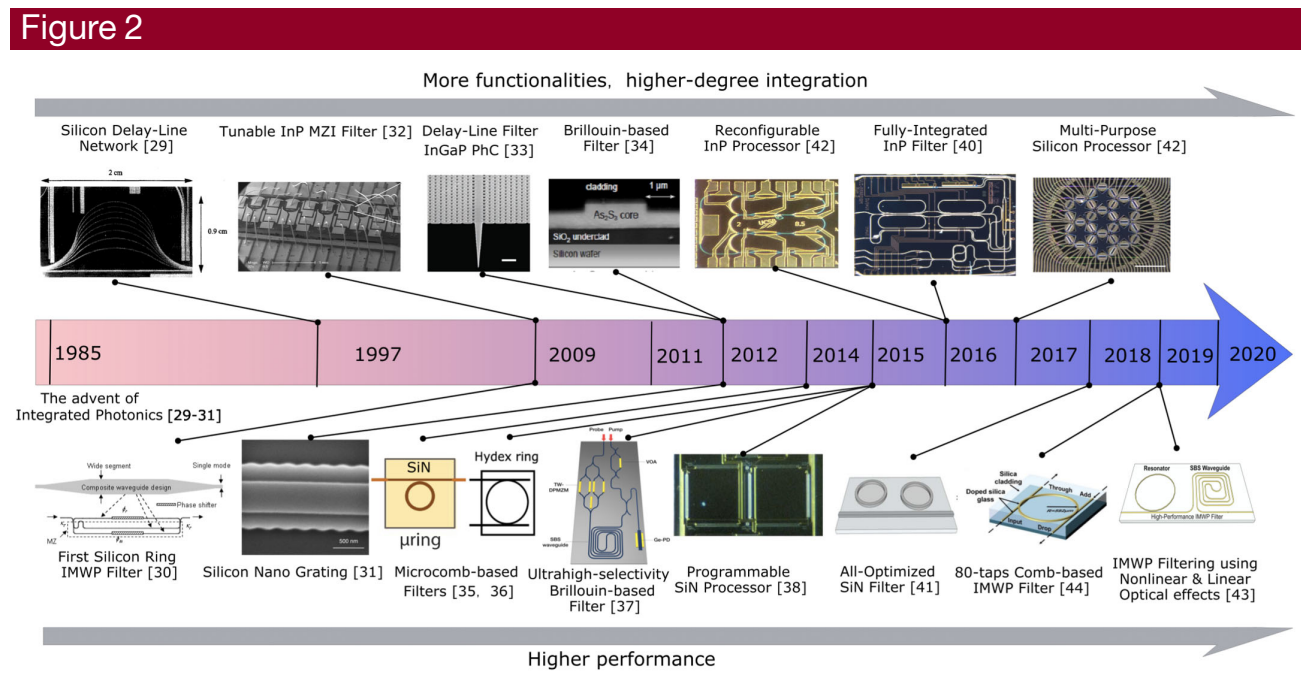

Timeline of major advancements in integrated microwave photonic filters, showing the milestones in architectures, platforms, and performance. Figures are reprinted from [29-44]. Reprinted with permission from Yegnanarayanan et al. Proc. SPIE 3160, 2-10 (1997) [29]. (C) SPIE. (C) 2009 IEEE. Reprinted, with permission, from Rasras et al., J. Lightwave Technol. 27, 2105-2110 (2009); Norberg et al., J. Lightwave Technol. 29, 1611-1619 (2011); Xue et al., J. Lightwave Technol. 32, 3557-3565 (2014) [30,32,35]. Reprinted with permission from [31,34,37,38,41]. Copyright 2012, 2015, and 2017 Optical Society of America. Reprinted from Sancho et al., Nat. Commun. 3, 1075 (2012); Liu et al., Nat. Photonics 10, 190-195 (2016); Fandiño et al., Nat. Photonics 11, 124-129 (2017); Pérez et al., Nat. Commun. 8, 636 (2017) [33,39,40,42]. Reprinted from Liu et al., APL Photon. 4, 106103 (2019); Xu et al., J. Lightwave Technol. 37, 1288-1295 (2019) [43,44]; licensed under a Creative Commons CC BY license. 
filters based on silicon and InP materials typically provide a spectral resolution of tens of gigahertz $(\mathrm{GHz})$ or several $\mathrm{GHz}$, which is mainly limited by the relatively high optical losses, as shown in Fig. 3(a). In order to reduce the optical losses, low-loss materials for photonic circuits are desired, which has motivated the development of SiN photonic circuits that can enable sub-GHz-level filter bandwidth. To achieve finer resolutions, the nonlinear optical SBS process in integrated photonic platforms, such as $\mathrm{As}_{2} \mathrm{~S}_{3}$ and silicon, offers a filter bandwidth down to several $\mathrm{MHz}$, with flexible bandwidth and frequency tunability. At the same time, a high stopband suppression of $>40 \mathrm{~dB}$ is desirable to effectively attenuate unwanted signals, which is challenging for integrated photonic filters. Recent progress has achieved ultradeep filter stopband rejection of $>50 \mathrm{~dB}$ using an RF-interference technique, which significantly outperforms filter schemes such as tapped-delay-line-based filters and spectrum transfer filters, as shown in Fig. 3(b). Comprehensive review and discussion of IMWP filter schemes that enable different features regarding spectral resolution and rejection will be carried out in this paper.

To date, advanced functionalities and footprint miniaturization have been widely demonstrated for IMWP filters with many impressive demonstrations. Equally important, the RF system performance of IMWP filters must ultimately match and even

\section{Figure 3}
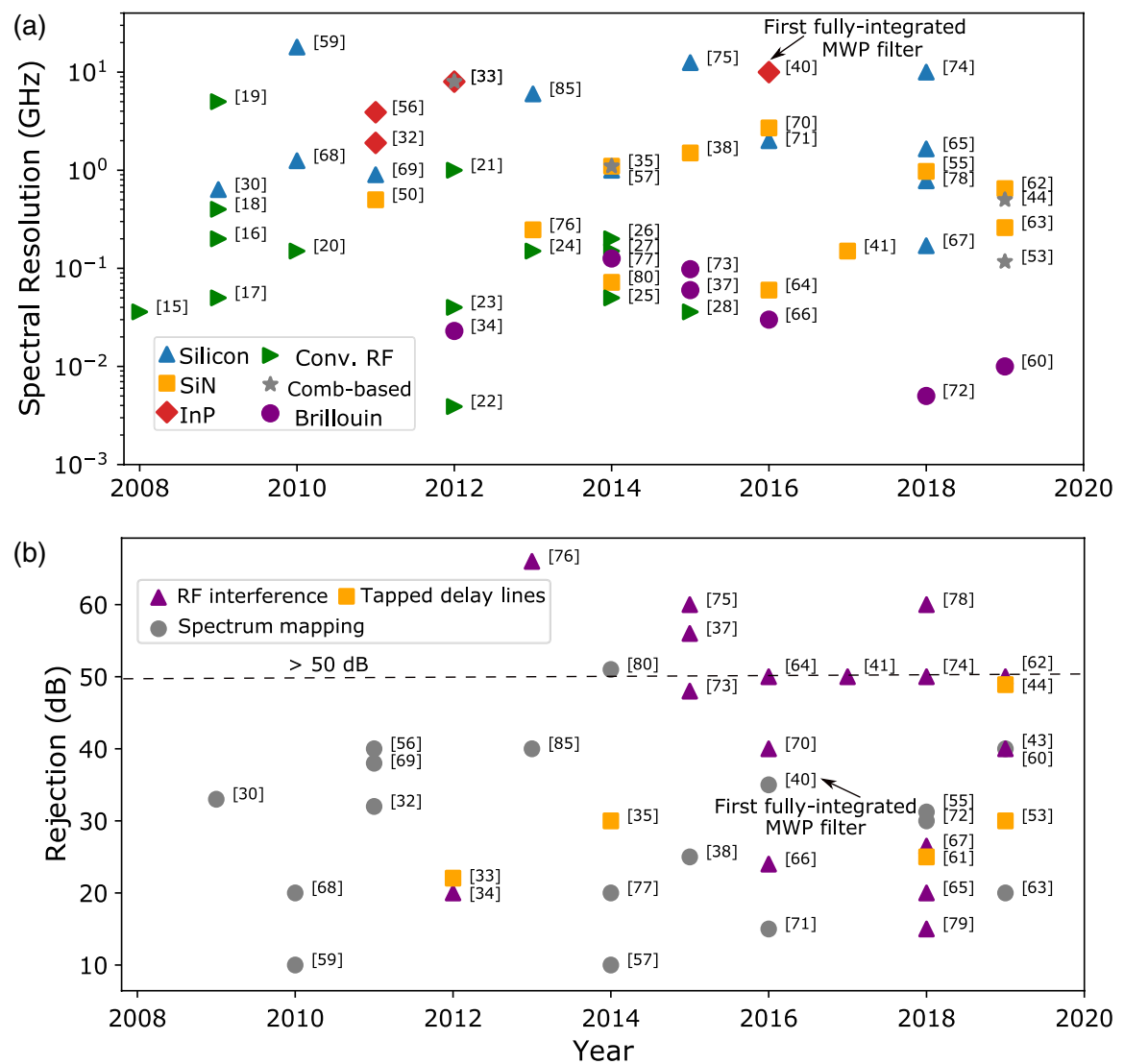

Evolution of filter spectral resolution and filter out-of-band rejection of IMWP filters. (a) Spectral resolution of IMWP filters based on different-material-based photonic devices and nonlinear optical effects (optical frequency combs and Brillouin scattering). (b) Filter rejection of IMWP filters implemented through various techniques. Conv. RF, conventional RF filters. Surveyed data for RF filters from [15-28] and for IMWP filters from [30,32-35,37,38,40,41,43,44,50,53,55-80]. 
surpass the conventional electronic counterparts, satisfying the performance requirements regarding RF link gain, noise figure, and dynamic range [12,81]. Therefore, the synergy of advanced functionality, photonic integration, and high RF performance must be addressed in future research and development of IMWP filters, if these technologies are going to be successfully deployed, as shown in Fig. 4. To sustain the signal quality, MWP systems need to meet the stringent figures of merit of RF link performance such as link gain, noise figure, and spurious-free dynamic range (SFDR), which indicates the RF signal loss, signal-to-noise (SNR) degradation, and signal distortions, respectively. These performance metrics, which have been widely studied and optimized for MWP links used for RF signal distribution and transport [82], must also be applied to IMWP filters, to ensure the overall system usability. For example, the signal of interest located within the filter passband is desired to undergo low RF insertion loss and low added noise, as shown in Fig. 1. So far, most of the reported IMWP filters have not addressed these demanding performances. The RF performance of IMWP filters is typically limited by the additional optical insertion losses of photonic circuits, suboptimal filter architectures, and device performance. Recently, impressive demonstrations were reported to show significantly improved RF performance of IMWP filters, achieving comparable performance metrics with conventional RF counterparts, in particular for the RF link gain as shown in Fig. 5. In these demonstrations, the increased RF performance was achieved using lowloss photonic circuits and novel filter schemes. These key performance metrics and state-of-the-art achievements will be discussed in this paper.

In this paper, we will review the basic principles of implementing IMWP filtering functionalities and summarize the key features of various approaches. With the basis of filter architectures being established, important integrated photonic devices used for IMWP filters will be reviewed, with emphasis on the emerging nonlinear optical material platforms that enable SBS and optical frequency combs. We then review the state-of-the-art performance of IMWP filters and highlight their technical advancements. Finally, we discuss the existing challenges and opportunities in the research and applications of IMWP filters.

\section{IMWP FILTER ARCHITECTURES AND FUNCTIONALITIES}

IMWP filters have been implemented using various schemes such as transverse filters based on multi-tap delay lines, coherent filters relying on spectrum mapping of optical filter responses, and the emerging RF-interference filters. These different filter schemes were realized using distinct filter architectures, exhibiting unique features of filter responses and performance. In this section, representative IMWP

\section{Figure 4}

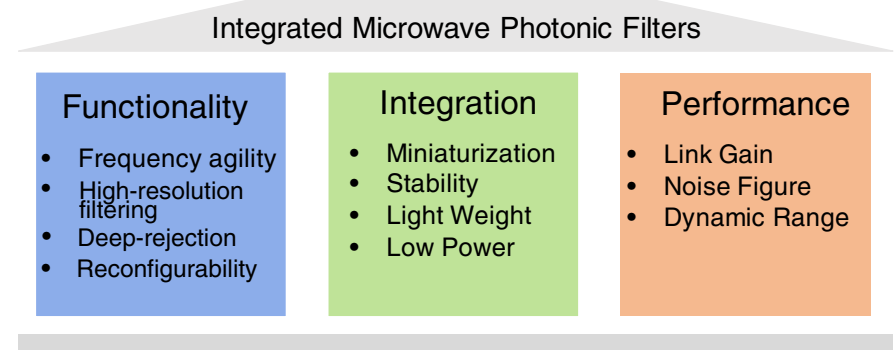

Functionality, integration, and performance are important metrics that underpin the integrated microwave photonic filters. 
filter schemes are reviewed, highlighting the recent advancements in implementing high-performance filtering.

\subsection{Typical Signal Modulation Schemes for IMWP Filters}

Signal modulation is a key part of an IMWP filter architecture, allowing for the signal upconversion from RF frequencies to optical frequencies, as shown in Fig. 1(b). The RF signal modulation can be realized by modulating the light source using E-O modulators, generating optical sidebands that carry RF signals. Different modulation schemes are able to alternate the properties of modulated optical signals, providing an important degree of freedom to implement various filter schemes.

Figure 6 shows the schematics of typical modulators and the associated optical spectra of modulated signals. The basic element of various modulators is the phase modulator that consists of a waveguide section with tunable phase in response to the applied electric field, due to the Pockels effect [83]. A phase modulation generates two balanced optical sidebands with a $\pi$ phase difference with respect to the optical carrier. By embedding the phase-variable segment in an interferometer topology, a MachZehnder modulator (MZM) $[84,85]$ can be constructed; the MZM is widely used in MWP applications. One of the unique properties of the intensity modulation is the inphase sidebands. Advanced and complex modulation formats can be achieved using a dual-parallel MZM (DPMZM). The structure topology of a DPMZM can be treated as two sub-MZMs incorporated in the two arms of a main MZM. Such a modulator can provide independent control of the amplitudes and the phases of the optical carrier and two sidebands, which forms the basis of the advanced MWP function implementations $[37,86]$. Complex modulations can also be achieved using other modulators such as the dual-drive modulator and polarization modulator. It should also be noted that external modulation has gained popularity due to the large modulation bandwidth and large modulation depth, compared to the direct modulation scheme [87].

Different types of modulations can be distinguished by examining the amplitude features and relative phase difference between the optical carrier and sidebands. For simplicity, we use a generic expression to describe the modulated optical fields, given by

$$
E_{\mathrm{mod}}=E_{c} \exp i\left(\omega_{c} t\right)+E_{l} \exp i\left[\left(\omega_{c}-w_{\mathrm{RF}}\right) t+\phi_{l}\right]+E_{u} \exp i\left[\left(\omega_{c}+\omega_{\mathrm{RF}}\right) t+\phi_{u}\right]
$$

\section{Figure 5}

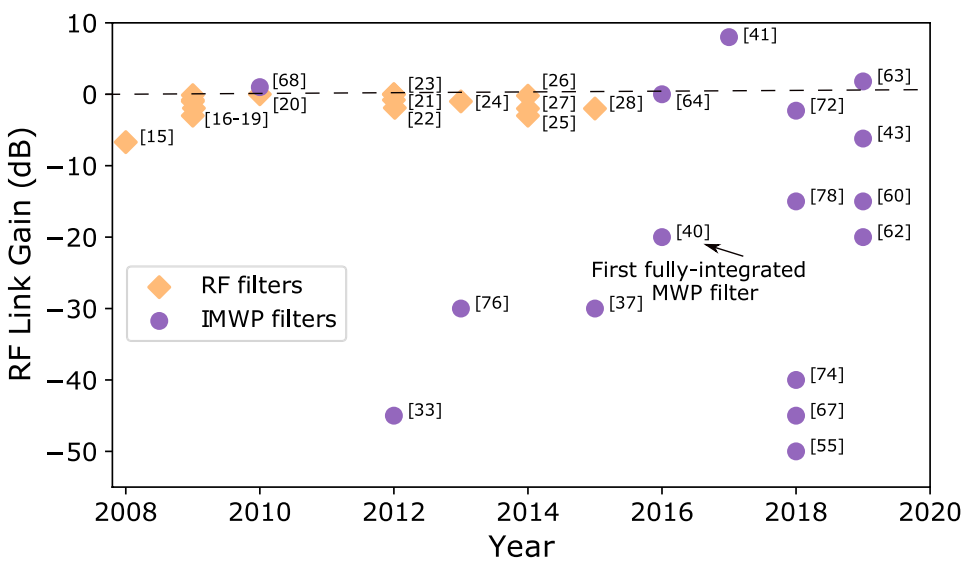

Comparisons of stopband suppression ratio of IMWP filters based on various techniques. Surveyed data for RF filters from [15-28] and IMWP filters from $[33,37,40,41,43,55,60,62-64,67,68,72,74,76,78]$. 
where $E_{c}, E_{l}$, and $E_{u}$ are the amplitudes of the optical carrier, the lower sideband, and the upper sideband; $\phi_{l}$ and $\phi_{u}$ are the phases of the lower and upper sidebands generated in the modulation, while we assume the carrier phase is zero. Here, we consider a small-signal model, so that we only focus on the first-order optical sidebands.

The commonly used modulation schemes for IMWP implementation can be categorized into four types, according to the amplitude and phase characteristics. Table 1 lists these modulation types including phase modulation, intensity modulation, and asymmetric dual-sideband modulations (Asym-DSB Type), as shown in Fig. 6, as well as the widely used single-sideband modulation (SSB). These modulation schemes play an important role in realizing different types of IMWP filters. In the following photonic filtering stage, integrated photonic circuits manipulate and tailor the amplitude and phase of the modulated optical spectrum. Eventually, the processed optical spectrum will be translated to desirable RF filter response via the photodetection process.

\section{Figure 6}
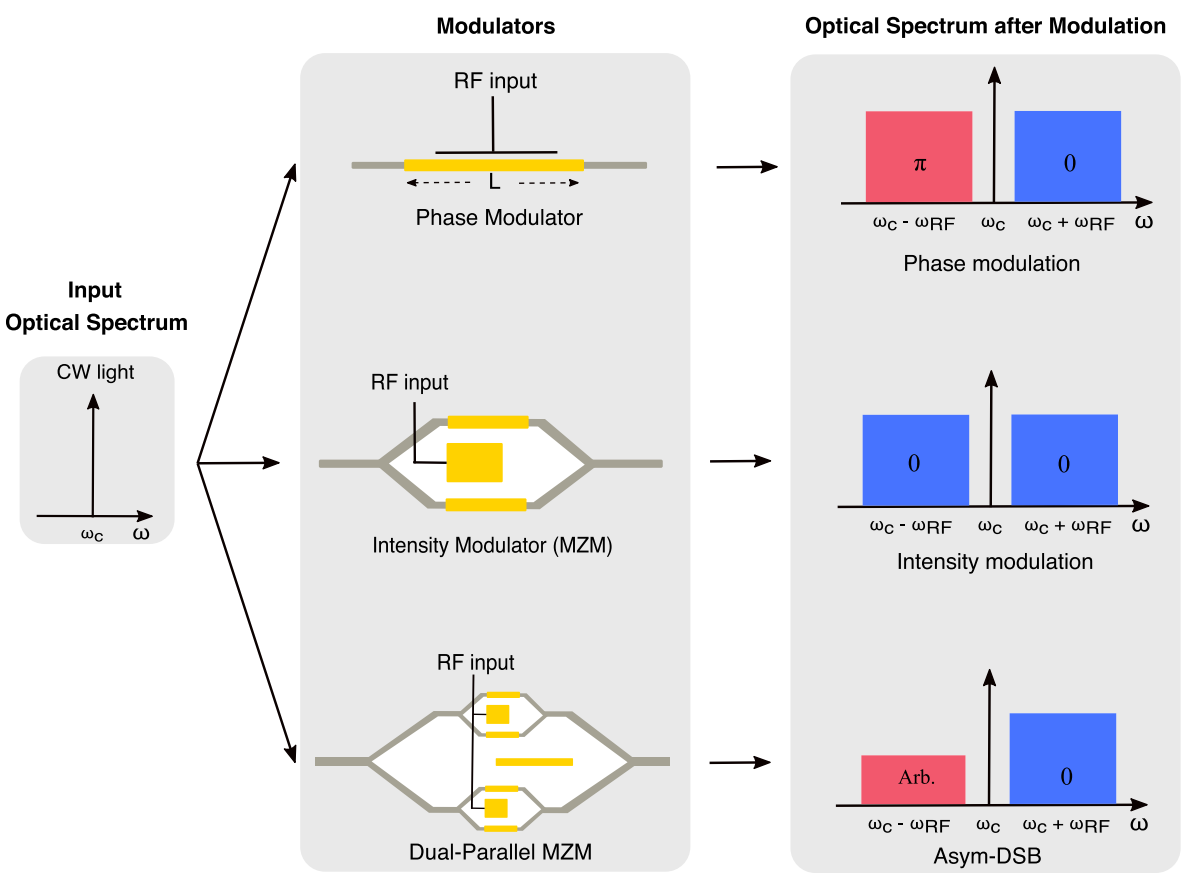

Typical E-O modulation formats for IMWP filter implementation. Corresponding optical spectra generated by (a) the phase modulator, (b) the intensity modulator, and (c) the dual-parallel MZM (DPMZM). The yellow segments in the modulators represent the phase modulation sections. The different colors of the sidebands indicate different phase relations. Arb, arbitrary phase.

Table 1. Amplitude and Phase Features of the Optical Carrier and Sidebands in Various Typical Modulation Schemes ${ }^{a}$

\begin{tabular}{|c|c|c|c|c|c|c|}
\hline & \multicolumn{2}{|c|}{ Lower Sideband } & \multicolumn{2}{|c|}{ Upper Sideband } & \multicolumn{2}{|c|}{ Carrier } \\
\hline & $E_{l}$ & $\phi_{l}$ & $E_{u}$ & $\phi_{u}$ & $E_{c}$ & $\phi_{c}$ \\
\hline $\mathrm{SSB}^{b}$ & 0 & 0 & $E_{u}$ & Arb. & $E_{C}$ & 0 \\
\hline Intensity Modulation & $E_{l}$ & 0 & $E_{u}=E_{l}$ & 0 & $E_{C}$ & 0 \\
\hline Phase Modulation & $E_{l}$ & $\pi$ & $E_{u}=E_{l}$ & 0 & $E_{C}$ & 0 \\
\hline Asym-DSB $^{c}$ & $E_{l}$ & Arb. $^{d}$ & $E_{u}$ & 0 & $E_{C}$ & 0 \\
\hline
\end{tabular}

${ }^{a}$ Annotations follow the parameters defined in Eq. (1).

${ }^{b}$ Single-Sideband.

${ }^{c}$ Asymmetric Dual-Sideband.

${ }^{d}$ Arbitrary. 


\subsection{Multi-Tap Filters}

Tapped delay-line filters, also termed as transverse filters, are the most conventional scheme to implement MWP filters $[6,88,89]$. The design of transversal filters was inspired by the digital filter concept that has been widely used in RF filters, based on well-developed discrete signal processing algorithms [90]. For a transversal filter, the input signal is discretely sampled, delayed, and weighted before being summed up. This process constructs a transfer function given by

$$
H(\omega)=\sum_{n=0}^{N-1} a_{n} e^{-j \theta_{n}} e^{-j n \omega \Delta T},
$$

where $a_{n}$ is the weight of the sampled signal in $n$th tap, $\theta_{n}$ is the carrier phase shift of the $n$th sample, and $\Delta T$ is the intertap signal time delay. According to the digital filter theory, each stage has a delay that is an integer multiple of the unit delay, i.e., $n \Delta T$. From Eq. (2), one key feature of this transfer function is the spectral periodicity, with a free spectral range (FSR) determined by the $1 / \Delta T$.

To implement transversal-type MWP filters, a transversal optical filter is embedded in an MWP link, as shown in Fig. 7, providing the desirable impulse response. From the calculation results in Figs. 7(b) and 7(d), the transfer function of the transversal filter (10 taps) can be changed by altering the values of $a_{n}, \theta_{n}$, and $\Delta T$, achieving arbitrary spectral responses and flexible tunability $[6,89,91]$. MWP filters with a limited number of taps are categorized to the finite-impulse-response (FIR) filter, while MWP filers with a large enough tap number approximating $+\infty$ refers to the infiniteimpulse-response (IIR) filter, which allows for a much narrower filter bandwidth and a higher extinction $[6,91]$.

Two typical configurations for implementing transversal MWP filters are shown in Figs. 8(a) and 8(b). The first type is based on a multi-tap delay-line network consisting of an array of optical delay lines between a signal splitter and a combiner. Each delay line is able to provide integer times of intertap signal delay, as well as amplitude and phase adjustment. The modulated optical signal is split into each sampling tap, before

\section{Figure 7}

(a)

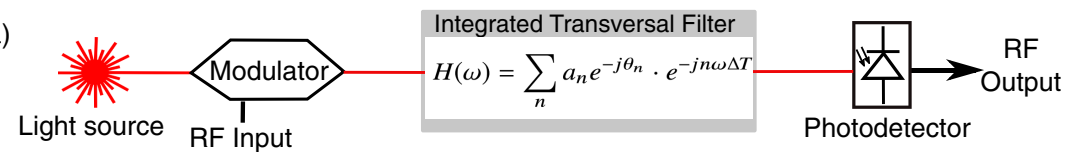

(b) Various $\Delta T$

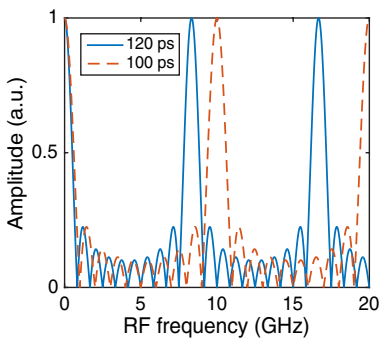

(c) Various tap numbers

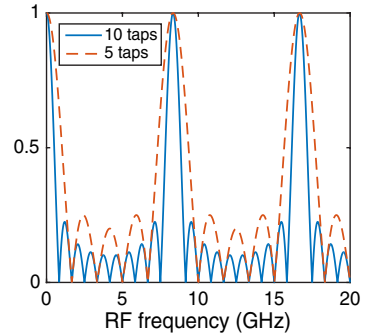

(d) Various $\theta_{n}$

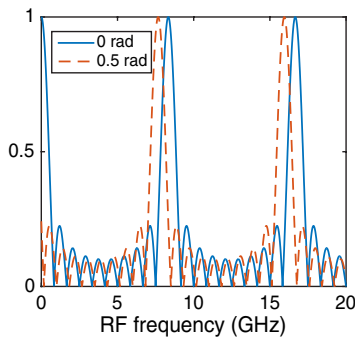

Multi-tap filter principle. (a) The architecture of an MWP filter based on the multi-tap configuration. The light source can be an incoherent broadband source or a laser array. (b)-(d) RF filter responses obtained by varying the value of the time delay, the total tap number, and the phase shift in Eq. (2). Reference responses in solid lines are obtained based on a 10-tap delay line network, uniform tap weights, 120 ps delay step, and zero phase offset. 
being combined together and detected by a photodetector. In contrast to the bulky fiber-based implementations [93-97], integrated optical devices such as delay-line network [29], subwavelength grating waveguide array [92], and cascaded ring network [50] can be used to implement compact MWP transversal filters with periodic frequency responses, as shown in Figs. 8(c)-8(f). The waveguide length precision, device robustness, and tunable elements make the integrated transversal MWP filter more stable against the environment fluctuations, which is a significant issue for fiber-based MWP transversal filters.

Another MWP transversal filter configuration is based on a spectral dispersion that provides different group delays for signals sitting at different optical frequencies [98-100], instead of using spatial delay lines with different waveguide lengths. In this scheme, an array of light sources is modulated to carry the same RF signals. After passing through the dispersive medium, signals at proper central frequencies, i.e., multiple tapped signals, acquire group delays with the same intertap time delay. A remarkable demonstration of an ultracompact MWP filter was achieved using on-chip

\section{Figure 8}

(a) Multiple Physical Delay Lines

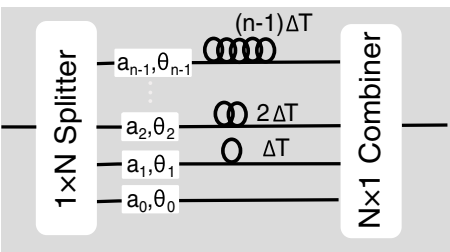

(c)

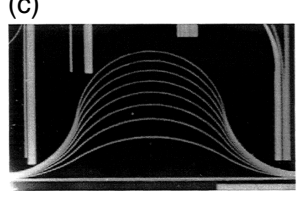

(d)

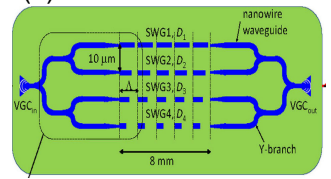

(e)

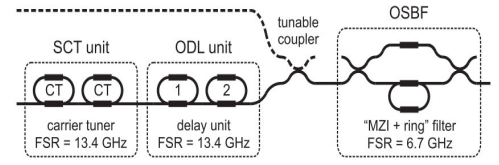

(f)

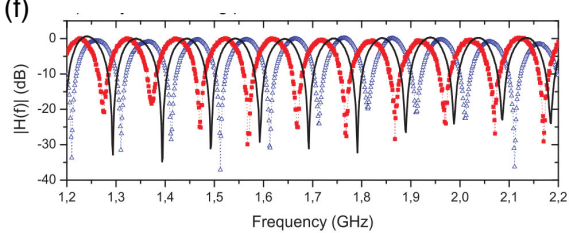

(b) Single Dispersive Element

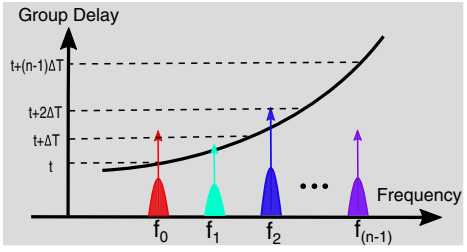

(g)

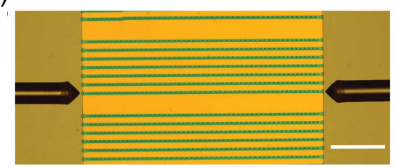

(h)

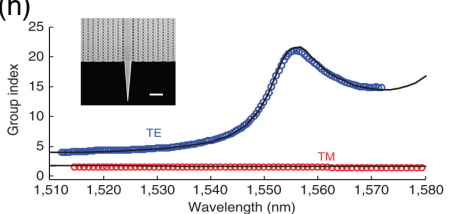

(i)

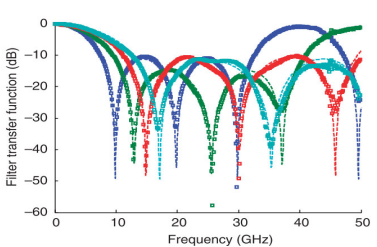

Schematics of integrated transversal MWP filters based on (a) tapped delay lines and (b) compact dispersive waveguides, respectively. Representative integrated delay-line-based devices including (c) delay-line network. Reprinted with permission from Yegnanarayanan et al., Proc. SPIE 3160, 2-10 (1997) [29]. (c) SPIE. (d) Subwavelength grating waveguide array. Reprinted from Wang et al., Sci, Rep. 6, 30235 (2016) [92]; licensed under a Creative Commons Attribution 4.0 International License. (e) Cascaded ring network with (f) measured filter responses when tuning the tap phase. Reprinted with permission from [50]. Copyright 2011 Optical Society of America. The demonstration using ( $\mathrm{g}$ ) chip-scale dispersive photonic crystal waveguides that (h) provides frequency-dependent group delay implements. (i) Tunable periodic RF filter responses. Reprinted from Sancho et al., Nat. Commun. 3, 1075 (2012) [33]. 
$1.5 \mathrm{~mm}$ long photonic crystal waveguide [Fig. $8(\mathrm{~g})]$ that provides a tunable and large group delay [Fig. 8(h)], exhibiting significant reduction in footprint and environmental sensitivity, compared with the fiber-based implementations $[98,100]$. However, these types of transverse filters are facing a common issue: the conventional multiwavelength light sources are bulky and can only provide limited number taps for GHz-level filter spectral resolution. Fortunately, this challenge can be overcome by the emerging optical frequency comb technology [101], which will be reviewed in detail in a later section in this paper.

A wide range of advanced and complex signal processing functions can be flexibly and dynamically constructed using the transversal filter configuration, in analogy with the versatile programmability of a discrete signal processing algorithm [90]. Functions such as differentiators [102,103], essentially complicated filters with unique amplitude and phase responses, have been achieved by configuring the weight, the phase, and the time delay of each delayed tap, as indicated by Eq. (2). Figure 9(a) illustrates a schematic of a reported integrated differentiator based on a four-tap optical delay line network [102]. By programming the attenuations and phase shifts, and thus the coefficients of each tap, a first-order optical field differentiator response can be implemented, exhibiting an amplitude response linearly proportional to the frequency detuning, as shown in Fig. 9(b). An analog differential computing operation can be performed, once a pulsed signal is processed by this differentiator, as shown in Fig. 9(c). A scalable and versatile approach to implementing IMWP differentiators was demonstrated using a multiwavelength light source based on microcombs [103], which provided a larger number of programmable optical taps, as shown in Fig. 8(d). In this demonstration, up to eight optical taps were generated from an optically pumped high-index doped silica glass microring resonator. Using a programmable spectrum shaper, the selected microcomb lines were weighted to implement tap coefficients for the desirable RF frequency-dependent responses [Figs. 9(e) and 9(f)], enabling an intensity differential operation function, as shown in Fig. 9(g). It should

\section{Figure 9}
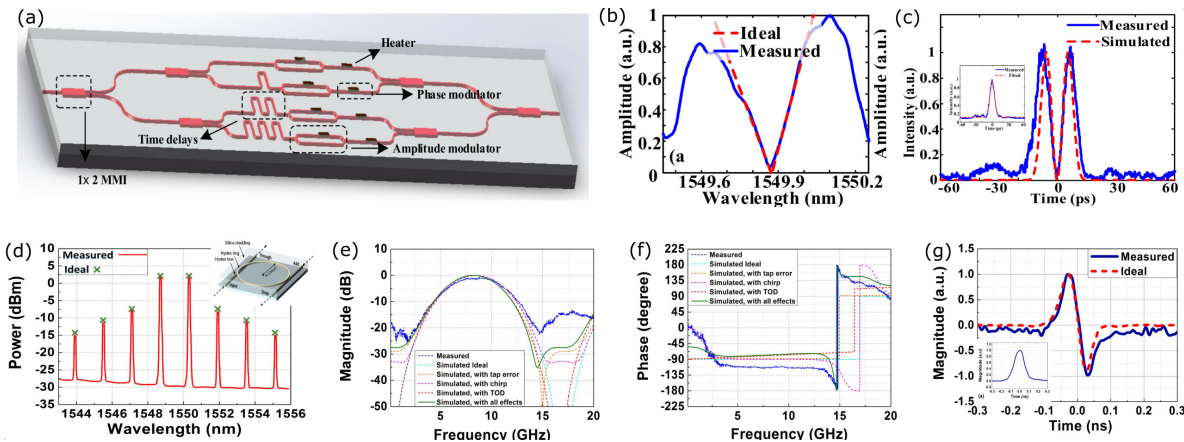

Complex signal processing functionalities enabled by transversal IMWP filter schemes. (a) The schematic of a field differentiator using a four-tap delay line network. (b) The spectral response of the first-order differential operation and (c) the temporal waveform after being applied the differentiator to a pulsed signal (shown in the inset). Reprinted with permission from [102]. Copyright 2015 Optical Society of America (d) The schematic of an intensity differentiator using multiwavelength optical source generated in a photonic microring. Copyright 2017 AIP Publishing LLC. Measured (e) amplitude responses and (f) phase responses of the first-order intensity differentiator. (g) The temporal RF waveform after the differential operation. Figures reprinted from Nguyen et al., Integrated Photonics Research, Silicon and Nanophotonics (IPRSN) (2015) [103]; licensed under a Creative Commons CC BY license. 
be noted that the above-mentioned differentiators can be both extended to achieve higher-order differential operations by adopting the targeted tap coefficients. With such a high-level flexibility and reconfigurability, Hilbert transformers were also reported using the transversal filter configurations [36,104,105]. Attractively, the ultrafast reconfigurability and function switching of the transversal filter can be activated by combining the ultrafast E-O elements such as phase modulators [100] and InP integrated pulse shapers [106], achieving tuning speeds of $\sim 40 \mathrm{~ns}$ and $\sim 400 \mathrm{~ns}$, respectively.

\subsection{Coherent Filter}

IMWP filters can be implemented in the coherent scheme using a single-wavelength optical source [91]. These types of coherent IMWP filters are generally constructed using a continuous-wave (CW) laser and a predefined on-chip optical filter with a desirable filter response. Without using the multi-tap configuration, the optical interference among different taps can be avoided in a coherent IMWP filter, resulting in improved stability and higher filter response design flexibility.

A typical configuration of this coherent MWP filter is shown in Fig. 10(a). The E-O modulation generates a modulated optical signal consisting of an optical carrier and sideband(s). An optical filter with a desirable filter response is used to coherently modify the sideband spectrum. In photodetection where the optical carrier and the processed sidebands mix, the optical filter response is consistently transferred to the RF domain, forming an RF filter response. In this MWP filter scheme, the RF filter response is simply defined by designing or tuning optical filter shapes of optical filters. On the other hand, the filter center frequency can be flexibly changed by altering

\section{Figure 10}

(a)
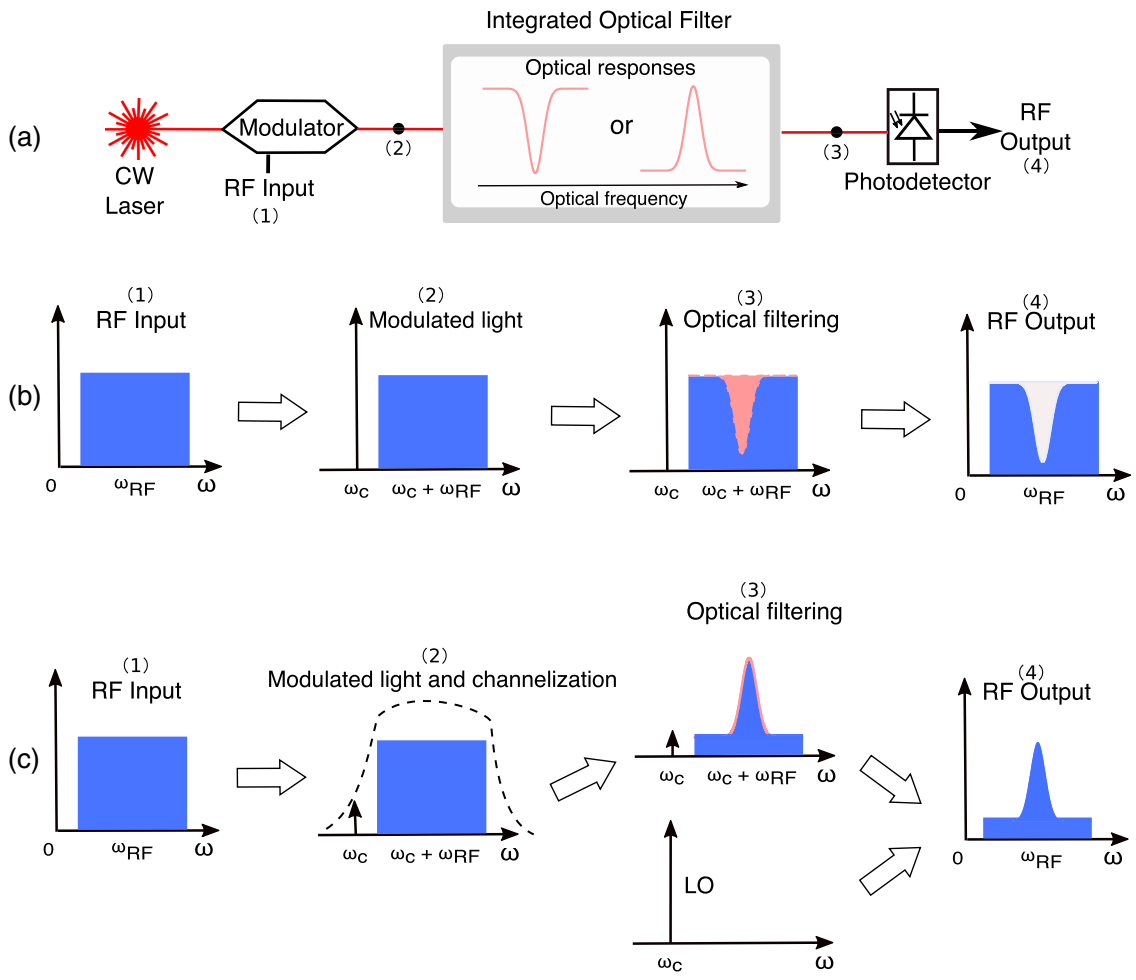

(a) Architecture of a coherent MWP filter scheme that relies on consistent optical-toelectrical spectrum mapping. Spectral illustrations at various locations along an MWP filter link to implement (b) a bandstop response and (c) a bandpass response, respectively. 
the optical carrier frequency or the optical filter central frequency. This is in contrast to the transversal MWP filter, in which complex and precise modification of time delay, phase, and weight coefficients of each tap is required for tuning filter shape and central frequency.

One of the simplest approaches to implementing an MWP filter is based on the SSB modulation and spectrum mapping, as shown in Figs. 10(b) and 10(c). The SSB modulation can be obtained by means of filtering out one of the optical sidebands or using advanced modulation schemes using DPMZM and DDMZM. In this filter configuration, the integrated optical response imposes a complex transfer function $H_{\text {opt }}(\omega)$ on the sideband, where $\omega=2 \pi f$ is the optical angular frequency. From Eq. (1) and Table 1, the processed SSB optical signal can be written as

$$
E_{\mathrm{proc}}=E_{c} \exp i\left(\omega_{c} t\right)+E_{u} \exp i\left[\left(\omega_{c}+\omega_{R F}\right) t+\phi_{u}\right] H_{\mathrm{opt}}\left(\omega_{c}+\omega_{\mathrm{RF}}\right),
$$

where $\omega_{c}$ is the optical carrier frequency, and $\omega_{\mathrm{RF}}$ is the input RF frequency.

Via direction photodetection, the processed optical signal is translated into photocurrent. Here, we focus on RF signal components at RF frequency $\omega_{\mathrm{RF}}$; the detected RF signals can be written as

$$
I_{\mathrm{det}, \mathrm{AC}}=2 \gamma_{P D} E_{c} E_{u} \cos \left[\left(\omega_{\mathrm{RF}}\right) t+\phi_{u}+\phi_{\mathrm{opt}}\right]\left|H_{\mathrm{opt}}\left(\omega_{c}+\omega_{\mathrm{RF}}\right)\right|,
$$

\section{Figure 11}

(a)

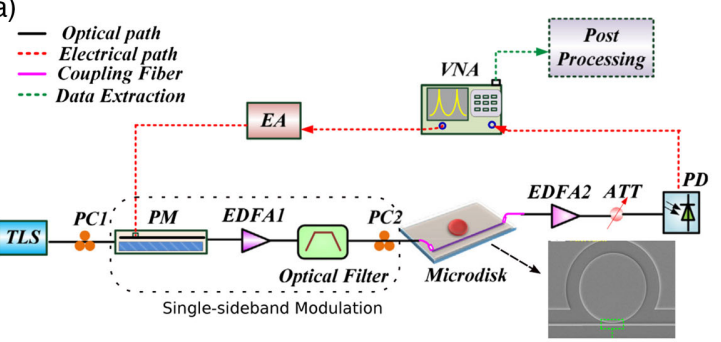

(c)
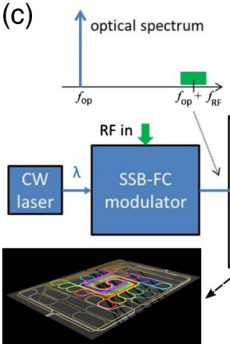
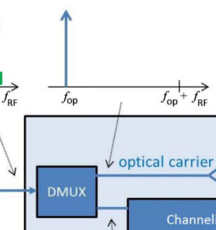
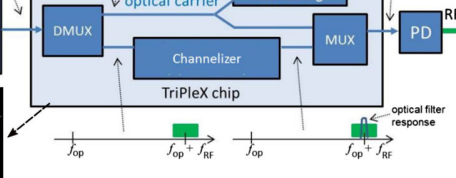

Representative demonstrations of chip-based MWP filters based on single-sideband modulation and spectrum mapping. (a) System configuration of an MWP notch filter using a silicon microdisk resonator and (b) the formed notch filter responses when tuning the laser frequencies. Reprinted from Opt. Commun, 335, Liu et al., "Photonic measurement of microwave frequency using a silicon microdisk resonator," 266-270, Copyright 2015, with permission from Elsevier [107]. (c) System configuration of an MWP bandpass filter using a $\mathrm{Si}_{3} \mathrm{~N}_{4}$ photonic circuit and (d) the tunable bandpass filter responses when tuning the laser frequency. In the bandpass filter scheme, the optical carrier is split into two arms, one for signal modulation and the other one for local oscillator. (c) 2014 IEEE. Reprinted, with permission, from C. Taddei et al., Microwave Photonics (MWP) and the 2014 9th Asia-Pacific Microwave Photonics Conference (APMP) 2014 International Topical Meeting on, pp. 44-47 (2017) [80]. 
where $\phi_{\text {opt }}$ is the phase response of the optical filter at the optical frequency of $\omega_{c}+$ $\omega_{R F}$. According to Eq. (4), the transfer function of the RF photonic filter is given by

$$
H_{\mathrm{RF}}\left(\omega_{\mathrm{RF}}\right)=\frac{2 \gamma_{P D} E_{c} E_{u} R_{\text {load }}}{E_{\mathrm{in}, \mathrm{RF}}}\left|H_{\mathrm{opt}}\left(\omega_{c}+\omega_{\mathrm{RF}}\right)\right| \exp i\left(\phi_{u}+\phi_{\mathrm{opt}}\right),
$$

where $R_{\text {load }}$ is the load resistance. From Eq. (4), the optical response will be one-toone mapped to the RF domain from the optical domain, preserving the same response in amplitude and phase. Hence, the design of the RF filter response can be eventually determined by the transfer function design of the optical filter.

A demonstration of a chip-based MWP notch filter using SSB modulation and spectrum transfer from an integrated silicon photonic microdisk resonator is shown in Figs. 11(a) and 11(b). The SSB modulation is formed by prefiltering the modulated optical signal prior to the on-chip optical sideband filtering, which forms an RF notch response with a bandwidth of more than $10 \mathrm{GHz}$. A chip-based MWP bandpass filter was demonstrated based on the same principle, as shown in Figs. 11(c) and 11(d). The implementation of the bandpass filter relies on the beat note of an auxiliary local oscillator (LO) and the sideband processed by an optical bandpass filter in photodetection, as shown in Fig. 10(c). In this scheme, sideband channelization and optical carrier splitting are required, which on the other hand increases the complexity of system architecture and optical filter design.

An alternative coherent scheme to generate a chip-based MWP notch filter is shown in Fig. 12(a). An integrated silicon photonic circuit consisting of four rings in an MZI architecture provides two optical notch responses symmetrically located around the optical carrier. These two notches are aligned to suppress the first-order optical sidebands with mirrored frequency offset. After photodetection, the suppressed frequency components from two sidebands superposed and formed an RF notch filter response [30]. The RF notch frequency is tunable by symmetrically altering the frequency separation of two optical notches. Using the same technique, chip-based MWP notch filters using various silicon photonic circuits were also demonstrated [58,108]. It should be noted the optical notch responses are desirable to produce null responses (close to ring resonator's critical coupling condition) at notch frequencies, which will

\section{Figure 12}

(a)

(b)
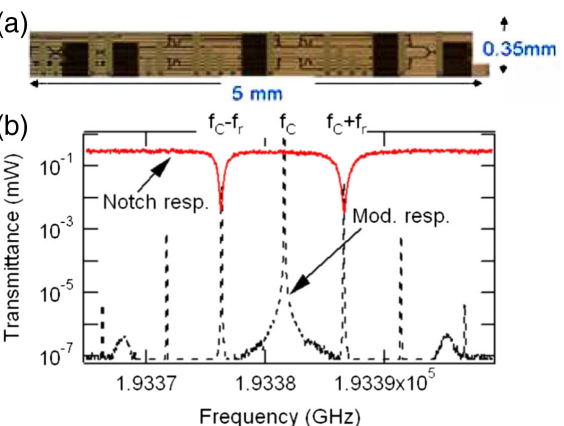

(c)

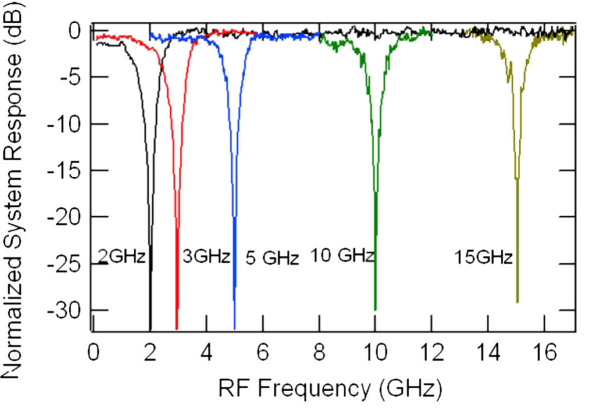

Chip-based MWP notch filter based on dual-sideband modulation and spectrum mapping. (a) An integrated silicon photonic circuit provides optical notch filter responses.

(b) Optical spectra of two optical notch responses (solid line) and the modulated optical signal (dashed line). Two optical notches are symmetrically distributed around the optical carrier and cancel the first-order sidebands generated by an intensity modulator. (c) Generated RF notch filter responses with notch frequency tunability. (C) 2020 IEEE. Reprinted, with permission, from Rasras et al., J. Lightwave Technol. 27, 2105-2110 (2009) [30]. 
be translated into deep RF notch responses. However, the design and control of an optical filter with null response at notch frequencies is very challenging. On the other hand, the filter bandwidth is lack of tunability while sustaining a deep notch, as the bandwidth and the suppression is usually coupled for ring resonators.

\subsection{RF-Interference Filter}

\section{4a. Asymmetric-Dual-Sideband-Based Processing}

To achieve an ultradeep filtering rejection, an emerging technique using Asym-DSB modulation has been extensively investigated [37,76,109-111]. The main feature of Asym-DSB modulation is that the two sidebands show an out-of-phase relation, as shown in Eq. (6) and Table 1. The underlying idea of this scheme is implementing a fully destructive interference at selective RF frequencies, forming an ultradeep suppression. Thus, the filter rejection is no longer determined or limited by the optical response of the optical filter. This unique scheme will eventually relax the requirements of optical filter design.

To gain more insights, we proceed to explain this scheme using mathematical descriptions, which will guide the design of novel RF photonic filters with enhanced properties. Here, we consider a case that an optical filter is used to process the upper sideband of the modulated signal based on the Asym-DSB modulation, as shown in Fig. 13. The processed optical field is given by

$$
E_{\mathrm{proc}}=E_{c} \exp i\left(\omega_{c} t\right)+E_{l} \exp i\left[\left(\omega_{c}-\omega_{\mathrm{RF}}\right) t+\pi\right]+E_{u} \exp i\left[\left(\omega_{c}+\omega_{\mathrm{RF}}\right) t\right] H_{\mathrm{opt}}(\omega),
$$

where the amplitudes of the lower sideband $E_{l}$ and the higher sideband $E_{u}$ can be different. $H_{\mathrm{opt}}(\omega)$ here and in following expressions stands for the optical transfer function at the optical frequency of $\omega_{c}$ at the optical frequency of $\omega_{c}+$ $\omega_{R F}$. The additional phase of $\pi$ of the lower sideband in Eq. (6) indicates the out-of-phase relation of two optical sidebands. This type of modulation can be produced typically using the aforementioned phase modulation with additional optical filtering to attenuate one sideband's amplitude. Alternatively, it can also be realized using complex modulation formats enabled by a DPMZM and a dual-drive MZM (DDMZM), with proper DC bias voltages applied to the modulators $[110,111]$.

Via direct photodetection, the detected RF signal at the RF frequency $\omega_{\mathrm{RF}}$ is given by

$$
I_{\mathrm{det}, \mathrm{AC}}=2 E_{u} E_{c} \cos \left(\omega_{\mathrm{RF}} t+\phi_{\mathrm{opt}}\right)\left|H_{\mathrm{opt}}(\omega)\right|-2 E_{l} E_{c} \cos \left(\omega_{\mathrm{RF}} t\right) .
$$

As shown in Eq. (7), the phase $\phi_{\text {opt }}$ of optical filter is transferred to the RF signal generated by the upper sideband, while the RF signal generated by the lower sideband does not acquire additional phase shift. The subtraction between these two terms indicates a destructive relation. For explicit descriptions, Eq. (7) can be transformed into a more compact form, given by

\section{Figure 13}
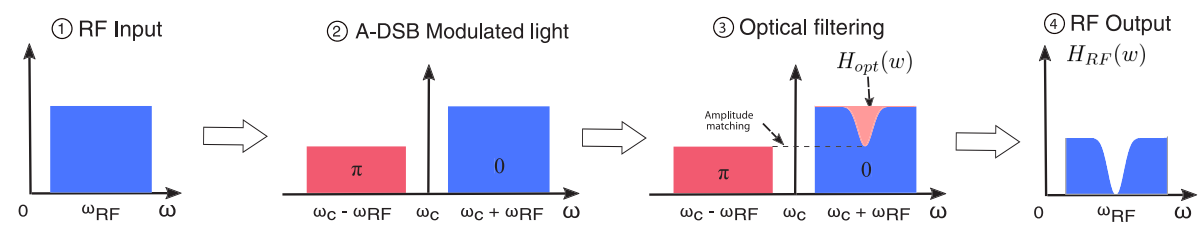

Schematic of an MWP notch filter based on asymmetric-dual-sideband (Asym-DSB) modulation scheme. 


$$
\begin{aligned}
I_{\mathrm{det}, \mathrm{AC}}= & 2 \gamma_{P D} E_{c} \sqrt{\left(E_{u}\left|H_{\mathrm{opt}}(\omega)\right|\right)^{2}+\left(E_{l}\right)^{2}-2 E_{u} E_{l}\left|H_{\mathrm{opt}}(\omega)\right| \cos \phi_{\mathrm{opt}}} \\
& \times \cos \left(\omega_{\mathrm{RF}} t+\phi_{\mathrm{RF}}\right),
\end{aligned}
$$

where the phase term $\phi_{\mathrm{RF}}$ is the resultant phase of the detected RF signal, expressed by

$$
\phi_{\mathrm{RF}}=\arctan \left[\frac{\sin \phi_{\mathrm{opt}}}{\cos \phi_{\mathrm{opt}}-\frac{E_{l}}{E_{u}\left|H_{\mathrm{opt}}(\omega)\right|}}\right] .
$$

From Eq. (8), the transfer function of the RF photonic filter based on Asym-DSB modulation scheme given by

$$
H_{\mathrm{RF}}(\omega)=\frac{2 \gamma_{\mathrm{PD}} E_{c} R_{\text {load }}}{E_{\mathrm{in}, \mathrm{RF}}} \sqrt{\left(E_{u}\left|H_{\mathrm{opt}}\right|\right)^{2}+\left(E_{l}\right)^{2}-2 E_{u} E_{l}\left|H_{\mathrm{opt}}(\omega)\right| \cos \phi_{\mathrm{opt}}} \exp i\left(\phi_{\mathrm{RF}}\right),
$$

where $E_{\mathrm{in}, \mathrm{RF}}$ is the input RF field and the transfer function here is defined as $H_{\mathrm{RF}}(\omega)=$ $I_{\text {det,AC }} R_{\text {load }} / E_{\mathrm{in}, \mathrm{RF}}$. The amplitude response of Eq. (10) is the basis of forming an RF notch filter with an ultrahigh rejection, leading to a simple relation described by

$$
\left|H_{\mathrm{RF}}\left(\omega_{\mathrm{RF}}\right)\right|= \begin{cases}0, & \text { if } \phi_{\mathrm{opt}}=0 \& E_{u}\left|H_{\mathrm{opt}}\left(\omega_{c}+\omega_{\mathrm{RF}}\right)\right|=E_{l} \\ \neq 0, & \text { otherwise }\end{cases}
$$

\section{Figure 14}

(a)

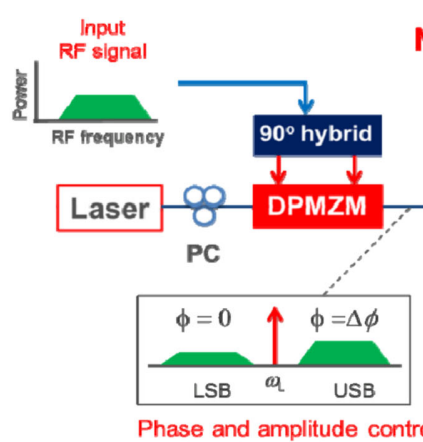

Novel MWP filter

Output

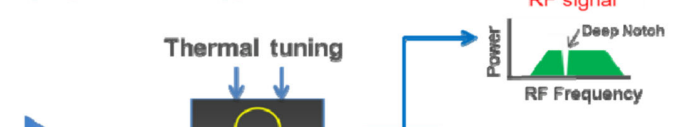

EDFA $\mathrm{PC} \quad \mathrm{Si}_{3} \mathrm{~N}_{4}$ ring

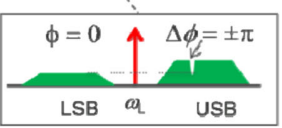

Phase and amplitude filter
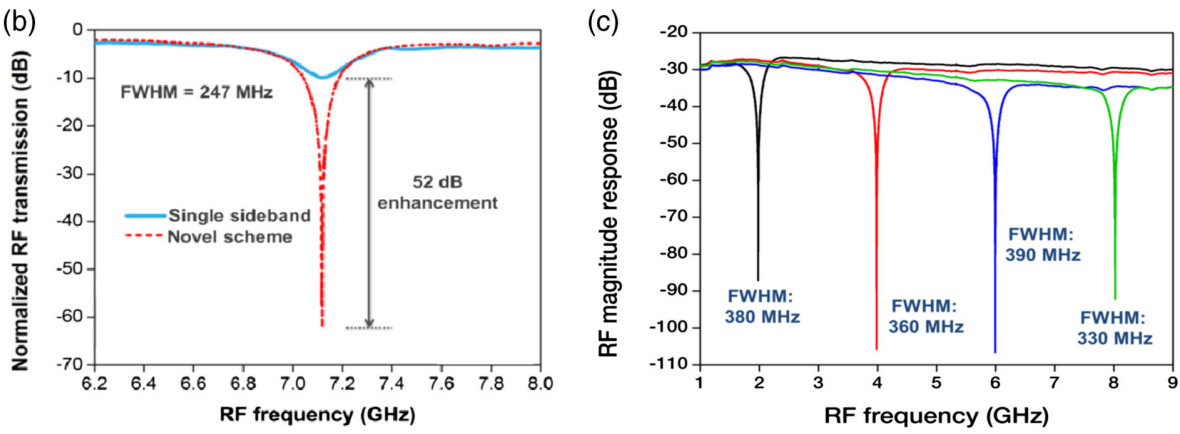

(a) Schematic of experimental implementation of a $\mathrm{Si}_{3} \mathrm{~N}_{4}$-ring-based MWP notch filter based on the Asym-DSB modulation scheme. (b) The comparison of RF notch filter responses using single-sideband modulation and the novel RF-interference scheme. (c) Measured frequency tuning of the MWP notch filter, showing a narrow bandwidth of $350 \mathrm{MHz}$ and ultrahigh rejection of $>55 \mathrm{~dB}$. Reprinted with permission from [76]. Copyright 2014 Optical Society of America. 
From Eq. (11), it is clear that a null response in amplitude can be formed at RF frequency $\omega_{\mathrm{RF}}$, if an optical filter provides a phase shift of $\phi_{\mathrm{opt}}=0$ and an amplitude response to balance the two sidebands at the frequency of interest.

Based on this novel scheme, a $\mathrm{Si}_{3} \mathrm{~N}_{4}$-chip-based MWP notch filter with an ultradeep rejection of $>60 \mathrm{~dB}$ was achieved, using a shallow optical resonance around $10 \mathrm{~dB}$ [76], as shown in Fig. 14(a). The Asym-DSB modulation scheme was generated using a DPMZM with appropriate DC bias voltages. An undercoupled ring resonator with an FWHM of $\sim 200 \mathrm{MHz}$ provides a shallow suppression in amplitude, which matches the amplitude of the lower optical sideband. Via photodetection, the RF cancellation gives rise to an ultradeep rejection in the RF domain, as shown in Fig. 14(b). This attractive feature significantly relaxes the optical filter design or additional tunable components, in contrast to the aforementioned filters relying on spectrum mapping. More strikingly, this scheme is able to decouple the RF filter rejection from the notch bandwidth, while sustaining flexible bandwidth tunability and frequency agility, as shown in Fig. 14(c). Moreover, the RF transfer function expressed by Eq. (10) also shows a very interesting feature in the resultant phase response, enabling the phase amplification effect that is extremely attractive for tunable RF photonic phase shifters and delay lines [112].

This Asym-DSB scheme also exhibits good compatibility with various optical filtering techniques [86,111], for example, the nonlinear optical effect, SBS [37]. The on-chip SBS process can also provide optical gain responses on one optical sideband with phase shift at the resonance frequency [37], which matches the amplitude of the other sideband with a $\pi$ phase difference, as shown in Fig. 15(a). The IMWP notch filter using a shallow SBS gain resonance showed impressive performance including $>50 \mathrm{~dB}$ rejection, sub-30-MHz spectral resolution, and ultrawide center frequency tunability of $>30 \mathrm{GHz}$ [37], as shown in Fig. 15(b).

A transformed realization of the Asym-DSB scheme was recently achieved using a ring resonator assisted by two cascaded MZIs [78], as shown in Fig. 16(a). In this approach, the SSB modulated signal is split to two branches with different power levels; the sideband of one branch is filtered by a ring resonator, and the other one remains unchanged. The relative phase between these two arms is tuned to be $\pi$,

\section{Figure 15}
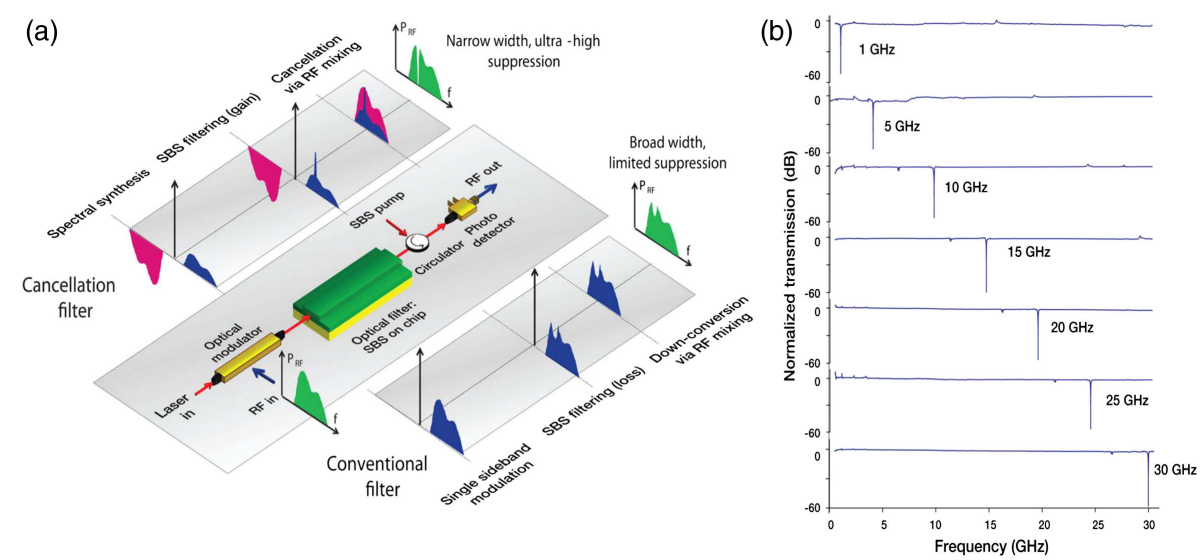

Schematic of SBS-chip-based MWP notch filter based on the Asym-DSB modulation scheme, compared to the filter based on single-sideband modulation scheme. (b) Measured stopband center frequency tuning over $30 \mathrm{GHz}$, maintaining a filter suppression of $>50 \mathrm{~dB}$ and a spectral resolution of $32 \mathrm{MHz}$. Reprinted with permission from [37]. Copyright 2015 Optical Society of America. 
leading to ultradeep RF notch responses due to the destructive interference, as shown in Fig. 16(b).

However, these advantages come with a vital side effect, the excess RF loss in the filter passband due to the inevitable wideband destructive interference. This side effect is attributed to the partial destructive RF interference induced by two out-ofphase sidebands. As a result, these types of chip-based MWP filters usually exhibit a unsatisfactory RF performance such as high RF link loss and high noise figure.

\section{4b. Symmetric-Dual-Sideband-Based Processing}

MWP filter schemes based on dual in-phase sidebands have recently gained great interest due to the potential of achieving strong filter passbands and the use of the conventional intensity modulation. Intensity modulation can produce two equalamplitude sidebands, with the same phases as the optical carrier, as shown in Table 1. To distinguish it from the above Asym-DSB modulation scheme, we define it as symmetric dual-sideband modulation (S-DSB). From a practical perspective, such a S-DSB modulation scheme is preferred, as it provides several advantages. First, the SDSB modulation can be directly generated through widely used intensity modulation and phase modulation. Second, in an intensity modulation and direct photodetection (IMDD) link architecture, the RF beat notes between the optical carrier and two inphase sidebands can add up constructively, forming strong RF signals compared to other cases. In a phase-modulation-based MWP link, high extinction filter response can form when the inherent destructive interference condition is disturbed. Third, one of the well-established link performance optimization techniques, for example, lowbiased MZM configuration, can be compatible with and applied to this filter scheme, allowing for filter performance enhancement.

Based on S-DSB modulation, MWP filters can be implemented by several configurations, depending on how the photonic processing is applied to the two optical sidebands. Here, we will introduce two types of configurations: 1) two sidebands are both optically processed; 2 ) only one sideband is optically processed.

Two-Sideband Processing. Here, we assume two optical filters are used to impose optical responses onto two in-phase optical sidebands, separately. The processed optical field is expressed by

$$
\begin{aligned}
E_{\mathrm{out}}= & E_{c} \exp i\left(\omega_{c} t\right)+E_{l} \exp i\left[\left(\omega_{c}-\omega_{\mathrm{RF}}\right) t\right] H_{\mathrm{opt}, 1}(\omega) \\
& +E_{u} \exp i\left[\left(\omega_{c}+\omega_{\mathrm{RF}}\right) t\right] H_{\mathrm{opt}, u}(\omega),
\end{aligned}
$$

\section{Figure 16}

(1)

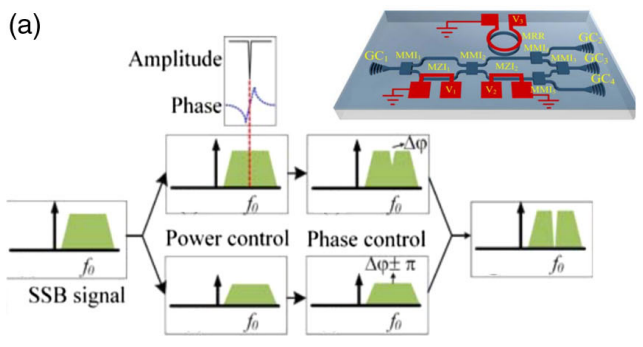

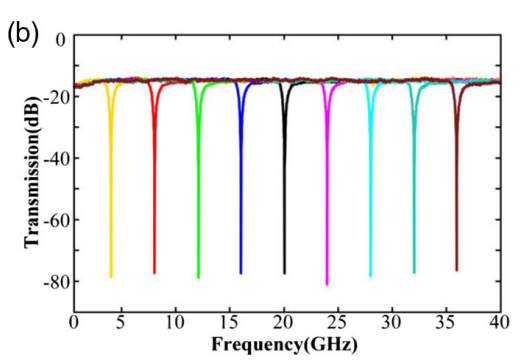

Schematic of an integrated MWP notch filter using an equivalent Asym-DSB modulation scheme. (a) The integrated photonic circuit consisting of an undercoupled microring resonator (MRR) assisted by two cascaded tunable Mach-Zehnder interferometers. (b) Measured RF notch filter responses centered at various RF frequencies. Reprinted with permission from [78]. Copyright 2018 Optical Society of America. 
where $H_{\mathrm{opt}, l}(\omega)$ and $H_{\mathrm{opt}, u}(\omega)$ are the optical transfer functions applied to the lower sideband and the upper sideband, respectively. Via direction photodetection, the detected RF components are given by

$$
I_{\mathrm{det}, \mathrm{AC}}=2 \gamma_{\mathrm{PD}} E_{0} E_{c} \sqrt{\left|H_{\mathrm{opt}, l}\right|^{2}+\left|H_{\mathrm{opt}, u}\right|^{2}+2\left|H_{\mathrm{opt}, l} H_{\mathrm{opt}, u}\right| \cos \Delta \phi} \cos \left(\omega_{\mathrm{RF}} t+\phi_{\mathrm{RF}}\right),
$$

where $\Delta \phi=\phi_{\mathrm{opt}, u}-\phi_{\mathrm{opt}, l}$ is the phase difference of two induced optical phase shifts while $\phi_{\mathrm{RF}}$ is the resultant RF phase shift. From Eq. (13), one can find that the null response at the frequency of interest can be achieved under two different conditions, expressed by

$$
H_{\mathrm{RF}}\left(\omega_{\mathrm{RF}}\right)= \begin{cases}0, & \text { if }\left|H_{\mathrm{opt}, l}\left(\omega_{c}-\omega_{\mathrm{RF}}\right)\right|=\left|H_{\mathrm{opt}, u}\left(\omega_{c}+\omega_{\mathrm{RF}}\right)\right|=0 \\ 0, & \text { or if } \Delta \phi=\pi \&\left|H_{\mathrm{opt}, l}\left(\omega_{c}-\omega_{\mathrm{RF}}\right)\right|=\left|H_{\mathrm{opt}, u}\left(\omega_{c}+\omega_{\mathrm{RF}}\right)\right| \neq 0 \\ \neq 0, & \text { otherwise. }\end{cases}
$$

In the first case, two optical responses are symmetrically located at each side of the optical carrier, with the same frequency spacing $\left(\omega_{\mathrm{RF}}\right)$ relative to the carrier frequency. Once the optical responses satisfy the condition of $\left|H_{\mathrm{opt}, l}\left(\omega_{c}-\omega_{\mathrm{RF}}\right)\right|=$ $\left|H_{\mathrm{opt}, u}\left(\omega_{c}+\omega_{\mathrm{RF}}\right)\right|=0$, the notch response can be synthesized at the RF frequency of $\omega_{\mathrm{RF}}$. This is the underlying idea of the very first IMWP notch filter demonstration using the coherent scheme [30], as illustrated in Fig. 12 in the previous section. In this demonstration, two optical filtering responses provided by two silicon ring resonators are desired to operate at the critical coupling condition, which on the other hand prevents the filter bandwidth tunability.

Alternatively, the second set of conditions provides a viable approach to achieving the RF notch response, without requiring ring resonators operating at the critical coupling condition. The principle is shown in Fig. 17(a). A recent demonstration of a chip-based MWP notch filter was achieved using two cascaded integrated ring resonators [41,113], as shown in Fig. 18(a). These two ring resonators are

\section{Figure 17}
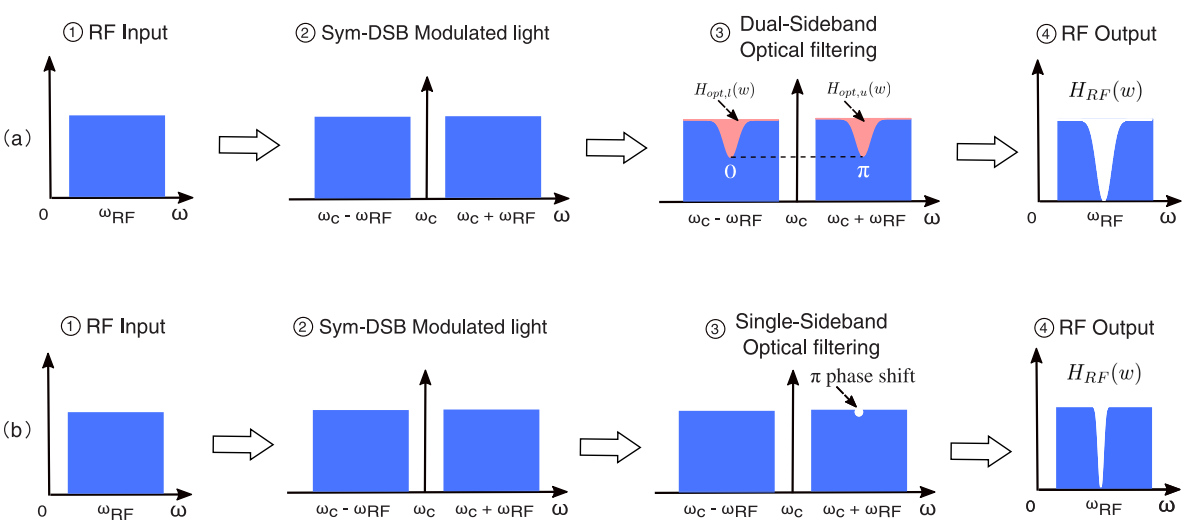

S-DSB-modulation-based IMWP filter implementation. (a) Schematic of RF photonic filter based on S-DSB modulation scheme, with two optical sidebands being processed. Two ring resonators operated in different coupling regimes are used to implement a set of conditions of $\Delta \phi=\pi$ and $\left|H_{\mathrm{opt}, l}\left(\omega_{c}-\omega_{\mathrm{RF}}\right)\right|=\left|H_{\mathrm{opt}, u}\left(\omega_{c}+\omega_{\mathrm{RF}}\right)\right|$. (b) Schematic of RF photonic filter based on SSB modulation scheme, with only one optical sideband being processed. A unique optical response is desired to satisfy the condition of $\phi_{\mathrm{opt}}=\pi \&\left|H_{\mathrm{opt}}(\omega)\right|=1$. 
operated in two distinct regimes, producing the same suppression in the amplitude with a completely different phase response. The undercoupled ring produces zero phase shift at the resonance frequency, while the overcoupled ring generates a $\pi$ phase inversion. The undercoupled resonator processes the lower optical sideband, while the overcoupled resonator processes the upper optical sideband. Two independent ring resonances are located symmetrically at both sides of the optical carrier. These unique and distinct features can exactly meet the conditions of $\Delta \phi=\pi$ and $\left|H_{\mathrm{opt}, l}\left(\omega_{c}-\omega_{\mathrm{RF}}\right)\right|=\left|H_{\mathrm{opt}, u}\left(\omega_{c}+\omega_{\mathrm{RF}}\right)\right| \neq 0$. Hence, a notch response can also be realized in the RF domain, showing an ultrahigh stopband suppression of $>50 \mathrm{~dB}$, as shown in Fig. 18(b). Positive RF link gain was able to achieved in the filter passbands, due to constructive RF interference and the link performance optimization technique compatible with the intensity modulation scheme. By employing multiple cascaded ring resonators, a dual-notch RF response was achieved, as shown in Fig. 18(c). In contrast to the spectrum transfer scheme, this RF-interference scheme is able to achieve filter bandwidth tuning, due to the fact that the deep suppression and the bandwidth tuning are decoupled properties, as shown in Fig. 18(d).

One-Sideband Processing. From the perspective of practical operation, it is preferable to optically process only one sideband of modulated optical signals. This will enable flexible frequency tunability of RF filter responses by only tuning the optical carrier frequency. This is in contrast to filters based on two-sideband processing, in which two independent optical filters have to be precisely tuned to frequencies located symmetrically around optical carrier. Due to the complexity in filter implementation and tuning, this dual-sideband-processing type of RF filter implementation has been less widely reported.

\section{Figure 18}
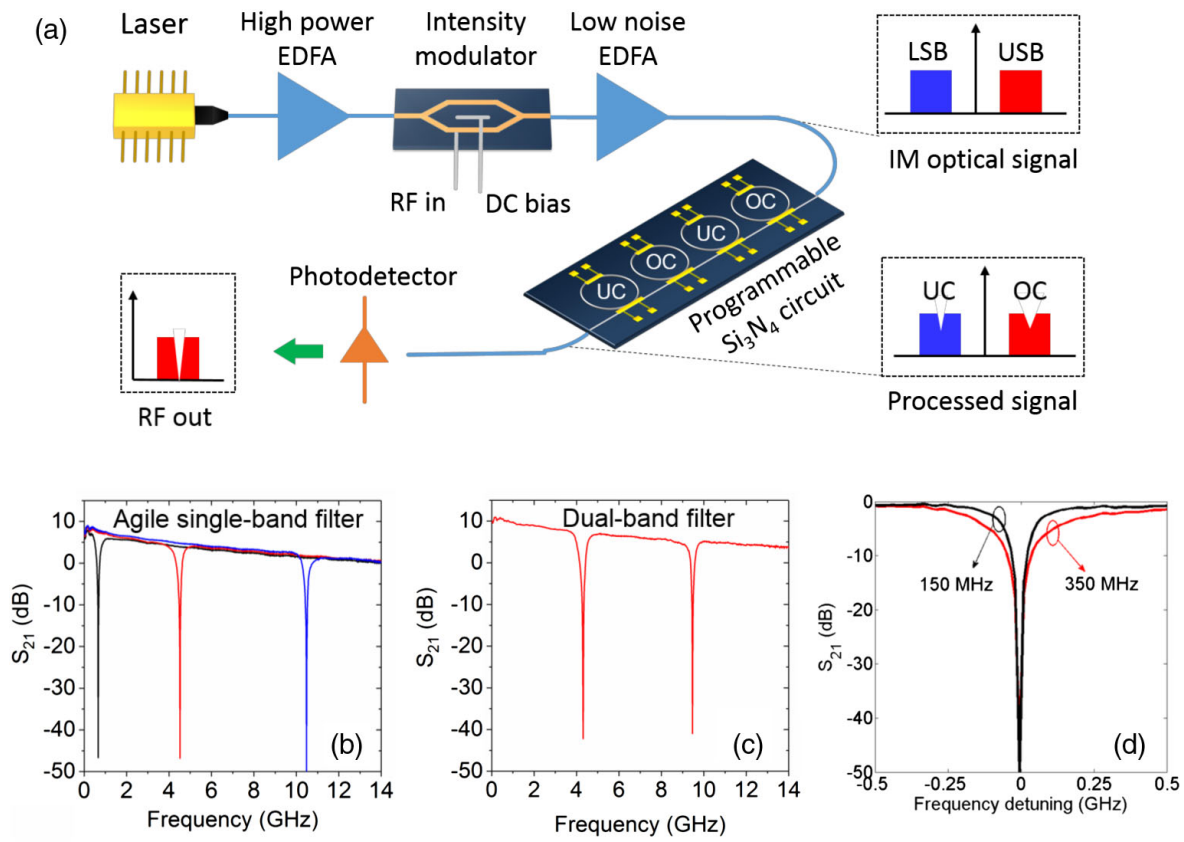

(a) Schematic of experimental setup of an integrated MWP filter with illustrative spectra at different locations along the link. LSB, lower sideband; USB, upper sideband; UC, undercoupled; OC, overcoupled. Spectra of (b) measured single-band filter at various RF frequencies, (c) measured dual-band filter, and (d) reconfigurable filter bandwidth. Reprinted with permission from [41]. Copyright 2017 Optical Society of America. 
Here, we introduce a design concept of an MWP filter based on optical processing of only one sideband of an intensity-modulated signal (Sym-DSB Type I, as listed in Table 1). To illustrate this filter scheme, we consider a case that the upper optical sideband is processed. Similarly, the detected RF response is expressed by

$$
H_{\mathrm{RF}}\left(\omega_{\mathrm{RF}}\right)=\frac{2 E_{0} E_{c}}{E_{\mathrm{in}, \mathrm{RF}}} \sqrt{1+\left|H_{\mathrm{opt}}\right|^{2}+2\left|H_{\mathrm{opt}}\right| \cos \phi_{\mathrm{opt}}} \cos \left(\omega_{\mathrm{RF}} t+\phi_{\mathrm{RF}}\right),
$$

where $H_{\mathrm{opt}}(\omega)$ is the applied optical response. Equation (15) implies the condition to implement a null response in the RF domain, given by

$$
H_{\mathrm{RF}}\left(\omega_{\mathrm{RF}}\right)= \begin{cases}0, & \text { if } \phi_{\mathrm{opt}}=\pi \&\left|H_{\mathrm{opt}}(\omega)\right|=1 \\ \neq 0, & \text { otherwise }\end{cases}
$$

Thus, the key to the implementation of an RF filter notch function is constructing a unique optical response that can satisfy $\phi_{\text {opt }}=\pi$ and $\left|H_{\text {opt }}(\omega)\right|=1$ at the notch frequency, as shown in Fig. 17(b). This desirable optical response is expected to produce a $\pi$ phase shift, while the amplitude response remains constant at the same frequency. However, to our best knowledge, there is no optical device that can directly generate such a unique optical response.

To bypass this limit, one possible approach is synthesizing the desired optical transfer function by cascading several transfer functions of different optical devices. This design idea can be mathematically described by

\section{Figure 19}
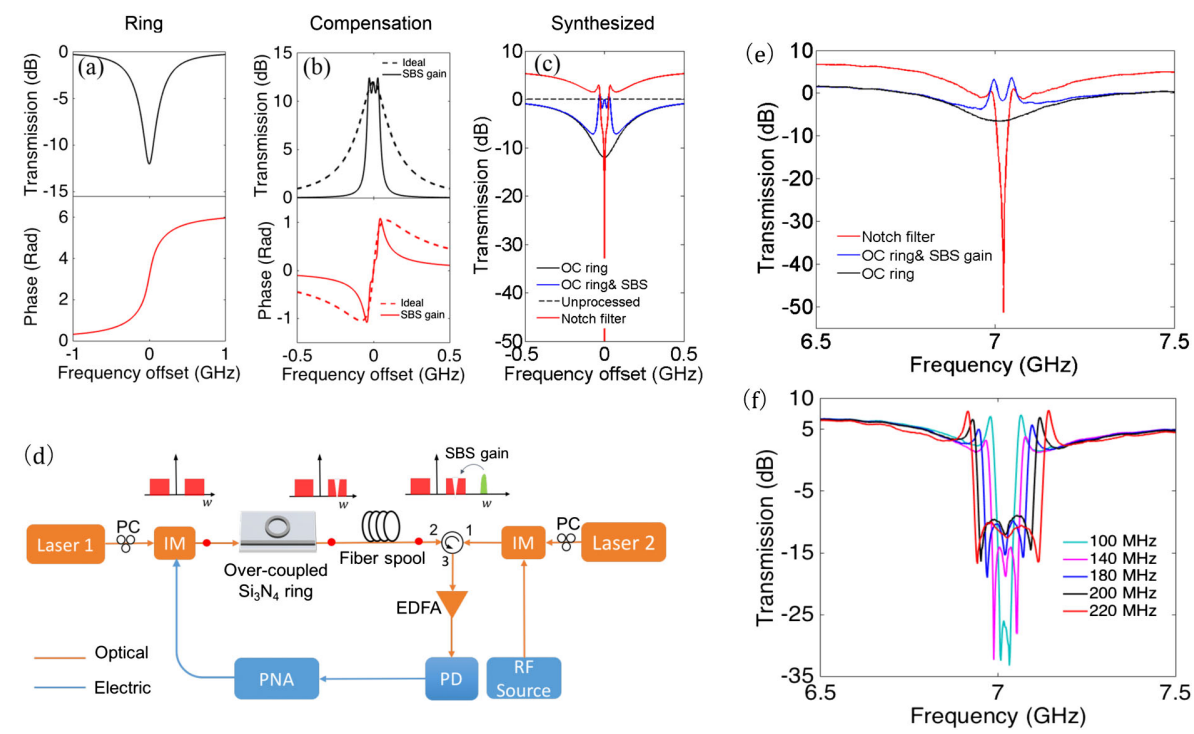

Simulations of the spectral responses of (a) an overcoupled (OC) ring with $400 \mathrm{MHz}$ linewidth, (b) ideal compensation transfer function and the approximate SBS gain resonance using three SBS pump lines, and (c) synthesized MWP notch filter with a 3-dB bandwidth of $60 \mathrm{MHz}$. (d) Schematic of the experimental setup. Qualitative optical spectra denoted by red points are shown above the setup diagram, respectively. (e) Measured MWP notch filter response, compared with the superposed response of the overcoupled ring and SBS gain. (f) Measured MWP bandstop filters with tunable 3-dB bandwidth. IM, intensity modulator; EDFA, erbium-doped fiber amplifier; PC, polarization controller; $\mathrm{PD}$, photodetector; VNA, vector network analyzer. Reprinted with permission from [64]. Copyright 2018 Optical Society of America. 


$$
H_{\mathrm{opt}}(\omega)=\prod_{i=1}^{n} H_{\mathrm{opt}, i}(\omega)=H_{\mathrm{opt}, 1}(\omega) \cdot H_{\mathrm{opt}, 2}(\omega) \cdots H_{\mathrm{opt}, n}(\omega)
$$

A feasible approach to achieving the desired optical transfer function is introducing a $\pi$ phase shift and then engineering the amplitude response. Based on this design principle, an MWP notch filter with deep stopband suppression, high RF link gain and tunable filter shape was recently reported, using the complementary optical responses of a passive ring resonator and active Brillouin gain resonance [64]. As shown in Fig. 19(a), an over-coupled ring resonator can provide such a $\pi$ phase shift for $H_{\mathrm{opt}, 1}(\omega)$. In contrast, the ring resonator produces a suppression in amplitude. SBS gain resonance $\left(H_{\mathrm{opt}, 2}(\omega)\right)$ is used to compensate the amplitude suppression, without affecting the $\pi$ phase, as shown in Fig. 19(b). By cascading these two transfer functions, a synthesized transfer function $H_{\mathrm{opt}}(\omega)=H_{\mathrm{opt}, 1}(\omega) \cdot H_{\mathrm{opt}, 2}(\omega)$ can

\section{Figure 20}
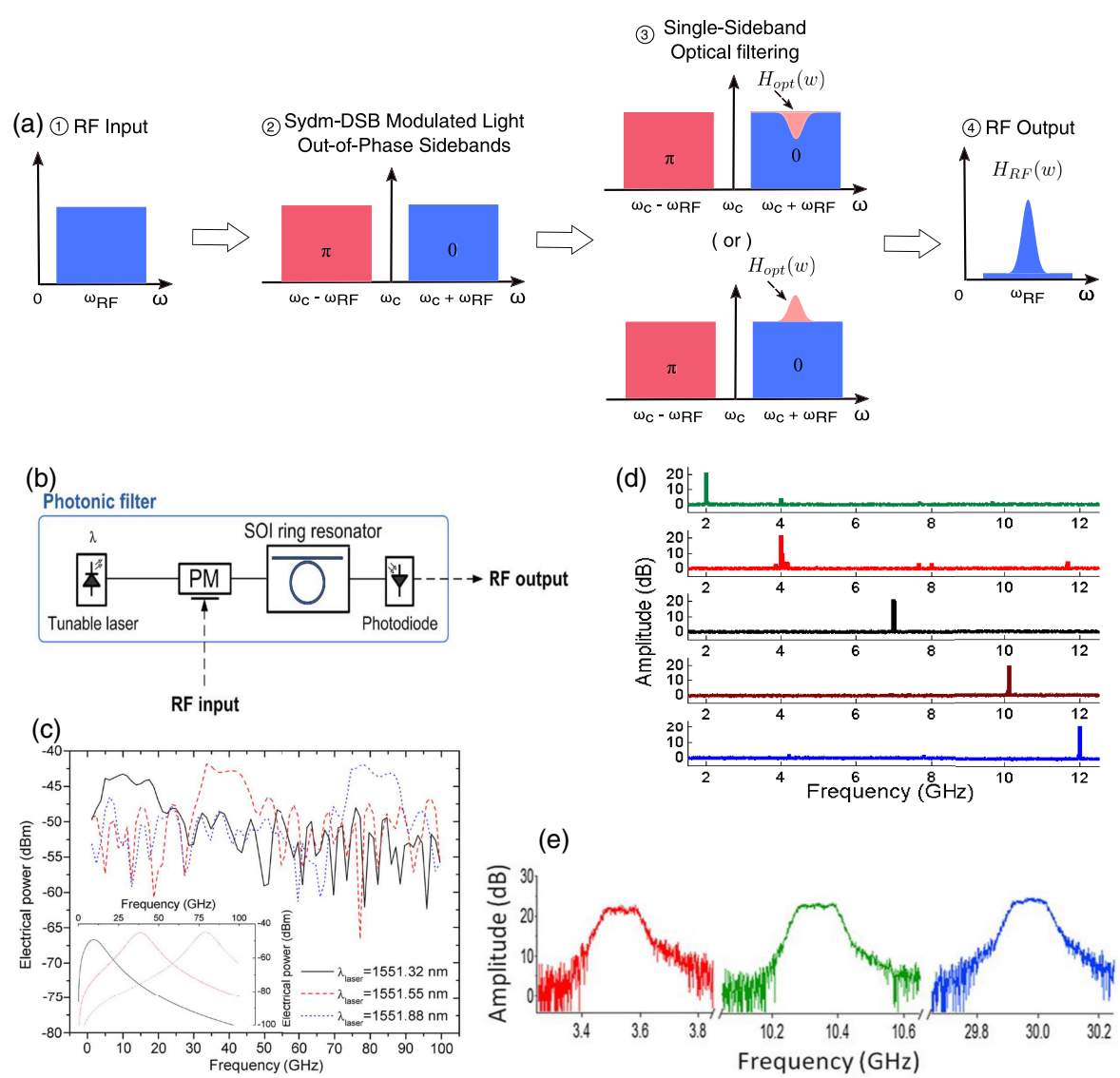

(e)

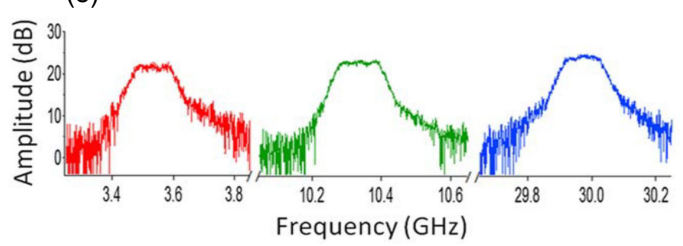

(a) Schematic of a bandpass MWP filter response based on the phase-modulation scheme and one-sideband processing. (b) Experimental setup and (c) the results of the first demonstration of the phase-modulation-based IMWP filter using an integrated silicon ring resonator. (c) 2019 IEEE. Reprinted with permission from Palací et al., IEEE Photon. Technol. Letter 22, 1276-1278 (2010) [59]. (d) Measured center frequency tunability of an on-chip Brillouin-based bandpass filter using the phase modulation. (e) Broadened filter bandwidth locating at a central frequency of 3.5, 10.3 , and $30 \mathrm{GHz}$ using broadband SBS gain response. Reprinted with permission from [34] Copyright 2012 Optical Society of America. (C) Reprinted with permission from [66]. Copyright 2016 Optical Society of America. 
be constructed, ideally exhibiting a flat amplitude and a $\pi$ phase shift, as shown in Fig. 19(c).

Figure 19(d) depicts the experimental setup for the novel MWP filter relying on the synthesized optical response. The intensity-modulated light was coupled into an over-coupled $\mathrm{Si}_{3} \mathrm{~N}_{4}$ ring resonator fabricated using low-loss TriPleX $\left(\mathrm{Si}_{3} \mathrm{~N}_{4} / \mathrm{SiO}_{2}\right)$ technology [51,114], which generates an optical notch response with a 3-dB bandwidth of $390 \mathrm{MHz}$ and a rejection of $10 \mathrm{~dB}$. The upper optical sideband was then processed by a tailored SBS gain resonance. The SBS gain process in an optical fiber can be optically tailored in both amplitude and bandwidth, which can selectively compensate the amplitude suppression induced by the overcoupled ring resonance. Moreover, the superposed optical response synthesizes a steeper resultant phase transition, while maintaining the phase shift of $\pi$ at the center frequency. Ideally, the synthesized optical all-pass filter response that exhibits a uniform amplitude response and frequency-dependent phase shift. Via direct photodetection, an RF notch filter with a stopband suppression of $\sim 55 \mathrm{~dB}$ and a spectral resolution of $\sim 60 \mathrm{MHz}$ was achieved, as shown in Fig. 19(e). By optically programming the SBS gain profile, rectangular-shaped bandstop RF filter responses were produced with a 3-dB bandwidth ranging from $100 \mathrm{MHz}$ to $220 \mathrm{MHz}$, as shown in Fig. 19(f). Attractively, the filter passband of $H_{\mathrm{RF}}(\omega)$ exhibits a strong strength, due to the constructive interference, in contrast to the filter scheme based on Asym-DSB modulation configuration. However, to further miniaturize this filter, the photonic integration of ring resonators and Brillouin-active circuits on the same chip is highly desirable [43].

Another ubiquitous MWP filter scheme is based on phase modulation (Sym-DSB Type II listed in Table 1) and one-sideband processing, as shown in Fig. 20(a). The underlying idea of this phase-modulation-based MWP filter is a reversed version of the former filter scheme, in which the out-of-phase sideband relation exists over a broadband instead of selective frequencies. In this case, when one of optical sideband is processed (attenuated or amplified), the unbalanced optical sidebands will lead to a partial RF cancellation in photodetection, while other frequencies components sustain the complete RF destructive interference. Such a difference in the degree of RF cancellation leads to the formation of a high-contrast RF filter bandpass response. This filter scheme has been widely implemented using fiber-based devices before the first demonstration using an integrated silicon ring resonator. The Fig. 20(b) shows the simple experimental setup for the first demonstration of this type filter scheme [59], producing RF bandpass filter response with a bandwidth of $>10 \mathrm{GHz}$, which is limited by the on-chip silicon ring performance. To enhance the RF passband strength, an intuitive way is to use amplification for the sideband processing, rather than the conventional ring-induced suppression. The use of on-chip SBS [115] is capable of amplifying (or de-amplifying) the optical sideband over a narrow frequency range (typically $30 \mathrm{MHz}$ ), providing high-extinction and narrow bandpass filter responses [34,60,116,117], as shown in Fig. 20(d). Broader RF bandpass filter responses $(>200 \mathrm{MHz}$ ) were subsequently achieved using broadened SBS gain response [66], as shown in Fig. 20(e).

\section{INTEGRATED PHOTONIC DEVICES AND EMERGING PLATFORMS FOR IMWP FILTERS}

To implement desirable RF filtering responses, a collection of integrated photonic devices are capable of providing various optical responses. This section will briefly introduce important passive integrated photonic devices, as well as the emerging nonlinear photonic circuits for IMWP filter implementation. In this section, we review important demonstrations of IMWP filters from a perspective of on-chip 
photonic device structures, available nonlinear optical effects, and photonic circuit architectures, which can potentially inspire the novel design of IMWP filters in the future. An excellent review of MWP filters from the perspective of material platforms can be found in Refs. [10,12,118].

\subsection{Photonic Devices based on Linear Optical Effects}

\section{1a. On-Chip Ring Resonators}

Ring resonators are one of the most important on-chip photonic devices for IMWP filters, due to its compact size, unique amplitude and phase response, and tunable properties [30,49,50,119-121].

An integrated ring resonator typically consists of a closed-loop optical waveguide that is coupled to a bus waveguide via evanescent optical fields, as shown in Fig. 21. The incoming light in the bus waveguide is coupled into the ring waveguide through the directional coupler. This optical coupler's properties are characterized by an amplitude coupling coefficient of $t$ and a transmission coefficient of $r$. At a particular optical wavelength, the coupled light is able to successively circulate in the looped ring waveguide. As a result, an enhanced optical field is built up inside the ring cavity, due to the constructive field interference effect. A comprehensive and insightful study of the resonator's principle and properties can be found in Refs. [121-123]. The fractional optical field transfer function is expressed by $[121,123]$

$$
H(\omega)=\exp [i(\pi+\phi)] \frac{a-r \exp (-i \phi)}{1-\operatorname{ar} \exp (i \phi)},
$$

where $\phi=k_{\text {neff }} L$ is accumulative round-trip phase shift, $k_{\text {neff }}=\frac{\omega}{c} n_{\text {eff }}$ is the effective optical wave vector in the ring waveguide, and $L$ is the ring circumference. $a$ is the amplitude attenuation given by $a^{2}=\exp \left(-\alpha_{\text {linear }} L\right)$ where $\alpha_{\text {linear }}$ is the linear optical loss coefficient.

By taking the square of the field transfer function presented by Eq. (18), a typical intensity transmission of a ring resonator exhibits periodic notch responses over the frequency. Figures 22(a) and 22(b) show representative optical responses of ring resonator operated in the under-coupled regime $(r>a)$ and the over-coupled regime $(r<a)$, respectively. The frequency spacing of two adjacent resonances is defined as free-spectral range (FSR). According to Eq. (18), the ring's optical response can be tuned by altering the coupling coefficients and the effective circulating phase, forming the basis of tunable filter responses and central frequencies.

More interestingly, the phase response shows completely different features in different coupling regimes, as shown in Figs. 22(c) and 22(d), respectively. These two

\section{Figure 21}

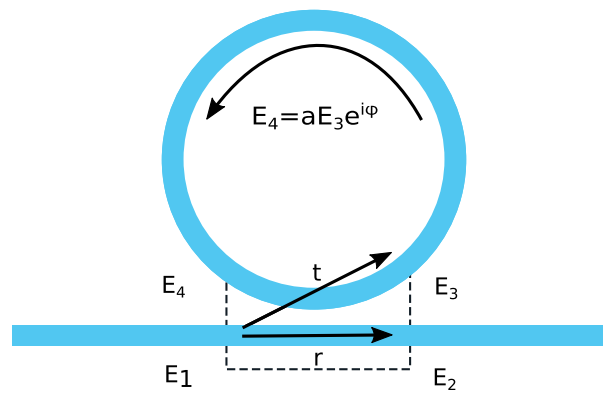

Schematic diagram of a ring resonator. The light is coupled from the bus waveguide to the ring resonator through the directional coupler denoted by the dashed box. 
distinct phase slopes show an opposite sign, while they have a similar rejection in transmission. Moreover, the under-coupled status provides a phase change around zero across the resonance frequency, while the over-coupled status produces a successive phase transition from 0 to $2 \pi$ around the resonance (unwrapped phase). Such $\pi$ phase inversion at the resonance frequency of the over-coupled ring resonator can allow for phase engineering for RF filter performance enhancement.

There have been many demonstrations of IMWP filter using on-chip microring resonators [30,41,58,65,68,74-76,113] and micro-disk resonators [107]. Most of the earlier silicon-ring-based MWP filters exhibit very coarse resolutions in the level of $\mathrm{GHz}$ or tens of $\mathrm{GHz}[58,65,75,107]$; this is mainly limited by the relatively high waveguide propagation loss of $3 \mathrm{~dB} / \mathrm{cm}$, according to Eq. (18). In order to increase the spectral resolution, multimode silicon waveguides with waveguide widths of $>2 \mu \mathrm{m}$ were used to reduce the optical propagation loss to a level of $0.1 \mathrm{~dB} / \mathrm{cm}$ [124-127]. Recently, an MWP bandpass filter with a minimal FWHM bandwidth of $170 \mathrm{MHz}$ was achieved, using a racetrack-type waveguide width of $2 \mu \mathrm{m}$ with a propagation loss of $0.25 \mathrm{~dB} / \mathrm{cm}$, as shown in Figs. 23(a)-23(c). The straight waveguides are designed to be multimodal to minimize the scattering losses induced by the sidewall roughness, while the waveguide bends are single-modal to reduce bend radius and ensure single optical mode operation. With these optimal designs, an IMWP bandpass filter with a minimal FWHM bandwidth of $170 \mathrm{MHz}$ was demonstrated, exhibiting a frequency tunability from $2 \mathrm{GHz}$ to $18.4 \mathrm{GHz}$, as shown in Figs. 23(d) and 23(e), respectively. The emerging ultralow loss materials such as $\mathrm{Si}_{3} \mathrm{~N}_{4}$ are also able to achieve sub-GHz-level filter resolution $[49,51,114,128]$. The extension of the number of filter passband resonators was recently reported using only one microring, while sustaining a high spectral resolution $(137.1 \mathrm{MHz})$ and achieving flexible filter

\section{Figure 22}
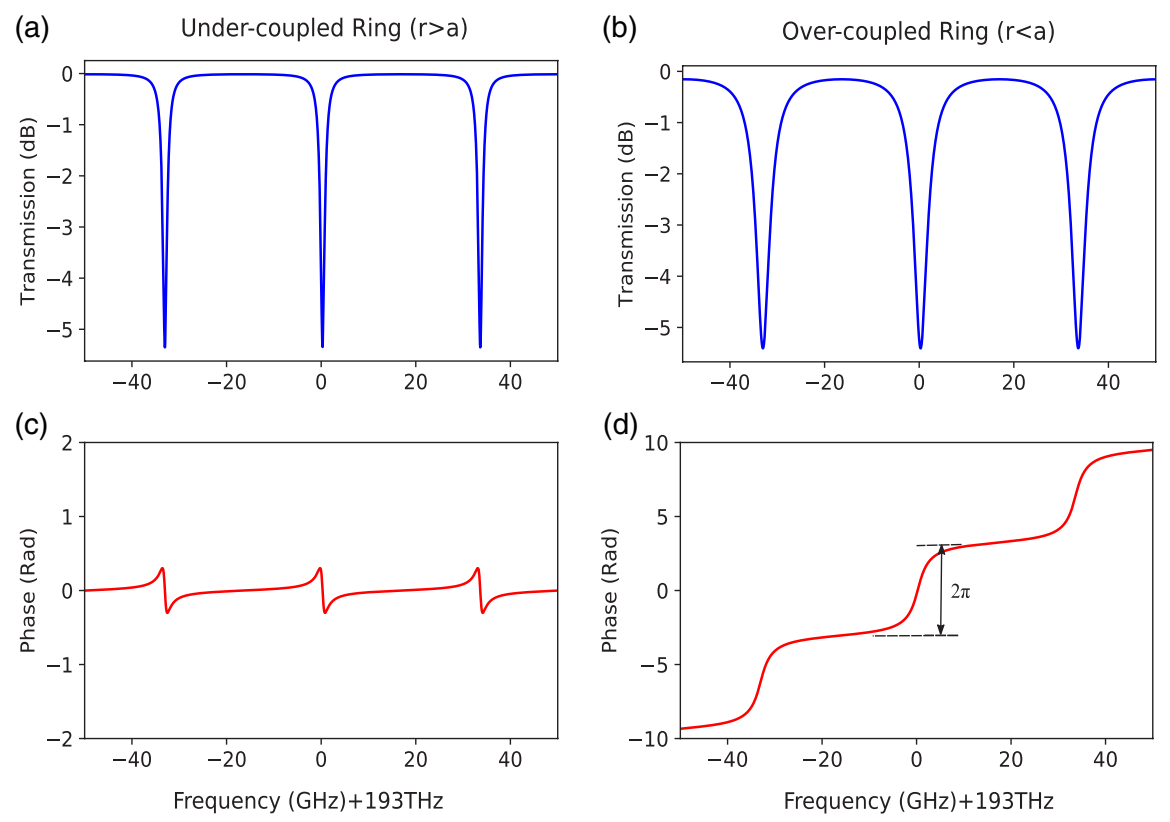

Simulated optical response of a ring resonator operated in (a) undercoupled regime when $a^{2}=0.187, r^{2}=0.5$; (b) the overcoupled regime when $a^{2}=0.187, r^{2}=0.06$. Parameters used in the simulation are $L=1 \mathrm{~mm}, \alpha_{\mathrm{dB}}=3 \mathrm{~dB} / \mathrm{cm}, n_{\mathrm{eff}}=3.0$, and optical wavelength near $1550 \mathrm{~nm}$. The phase response over the frequency in two coupling regimes shows an opposite sign, enabling negative and positive group delay, respectively. 
response tuning [79]. This filter scheme is implemented using the orthogonally polarized optical modes in a high-index doped silica glass microring resonator. Based on the coherent single-passband filter scheme, filter responses in two polarizations can add up to form a dual-passband RF filter response. The strength of each passband can be flexibly tuned by adjusting the relative projection of the input optical signals to each polarization of microring resonance.

The use of multiple on-chip ring resonators provides a versatile way to implement IMWP filters. One of the multi-ring architectures is side-coupled integrated spaced sequence of resonators (SCISSORs) $[129,130]$. The SCISSORs structure is able to cascade the optical responses of multiple rings to synthesize a broader-band response, or to use rings to process different signal components [70,131,132]. $\mathrm{A} \mathrm{Si}_{3} \mathrm{~N}_{4}$-chipbased MWP filter with reconfigurable responses was recently demonstrated using a SCISSORs-type structure consisting of three rings with different circumferences [70], as shown in Fig. 24(a). The first ring resonator was operated in the strong overcoupled regime to produce a shallow amplitude suppression and a large phase shift across the resonance frequency. This large phase shift can induce a phase shift of $\pi$ onto the optical carrier, which can flexibly transform the modulation scheme between phase modulation and intensity modulation, without replacing actual modulators, as shown in Fig. 24(b). Another two identical ring resonators with shorter lengths were used to process the two optical sidebands, respectively, with slightly different frequency offsets from the optical carrier frequency. Following the similar principle of MWP filters using a dual-sideband scheme in Subsection 2.4b, RF passband and stopband responses with frequency tunability can be implemented via the RF interference in photodetection, as shown in Figs. 24(c) and 24(d). By altering frequency offsets, the filter shape and bandwidth can be flexibly tuned, as shown in Figs. 24(e) and 24(f).

Coupled-resonator optical waveguides (CROWs) are an alternative arrangement to enhance the filtering functionality, which was experimentally demonstrated [133-135]. According to the theoretical study, the CROWs are able to implement higher-order filter responses with a flattop and a high out-of-band extinction ratio

\section{Figure 23}

(a)
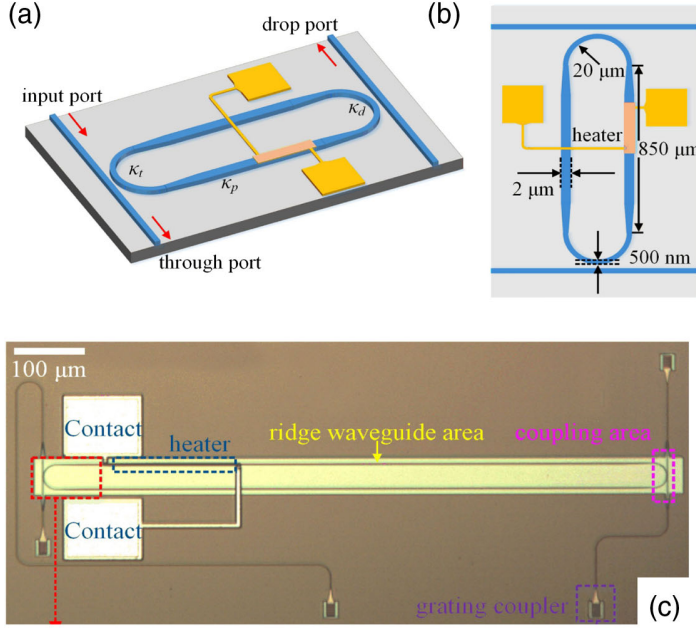

(b)

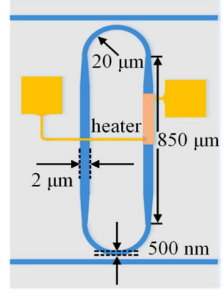

(e)
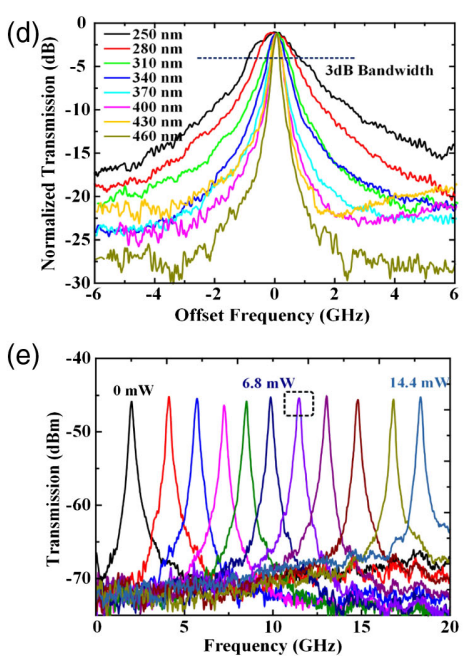

(a) Layout of the ultrahigh- $Q$ microring resonator. (b) Schematic top view of the microring resonator. (c) Microscope image of the microring resonator. (d) Filter bandwidth tunability from $170 \mathrm{MHz}$ to $1.7 \mathrm{GHz}$. (e) Filter central frequency tunability from $2 \mathrm{GHz}$ to $18.4 \mathrm{GHz}$. (c) 2010 IEEE. Reprinted with permission from Qiu et al., J. Lightwave Technol. 36, 4312-4318 (2010) [67]. 
[136]. A representative implementation of CROW-type resonators is shown in Fig. 25(a), consisting of several mutually coupled silicon resonators [137]. As shown in Fig. 25(b), the increase in the number of coupled resonators will generate optical filter responses with a sharper filter roll-off, compared to a single ring resonator response. Using low-loss silicon shallow-ridge waveguides, a bandpass filter response with $53 \mathrm{~dB}$ extinction ratio and $1.9 \mathrm{GHz}$ spectral resolution was achieved, which is suitable for microwave filtering applications, as shown in Fig. 25(c). Using ultralowloss $\mathrm{Si}_{3} \mathrm{~N}_{4}$ waveguides [138], a filter based on three-order CROWs was recently demonstrated to possess an ultrahigh out-of-band rejection of $80 \mathrm{~dB}$ and a 3-dB bandwidth of $1.6 \mathrm{GHz}$, as shown in Figs. 25(d)-25(g). Using the active InP-InGaAsP material platform, the coupling coefficients and the resonance frequencies of CROWs can be independently and precisely tuned [56], as shown in Figs. 25(h) and 25(i). Such a flexible tunability enabled a filter passband bandwidth from 3.9 to $7.1 \mathrm{GHz}$, with high-level central frequency agility and filter response reconfigurability, as shown in Figs. 25(j) and 25(k).

One of the common issues for ring-based IMWP filters is the limited center frequency tuning range constrained by the periodic optical responses of ring resonators. One feasible solution to relax this limitation is using a parallel-coupled ring-resonator filter scheme $[139,140]$. By using the Vernier effect, an enhancement of FSR by 3 or 4 times was achieved with improved filter roll-off and passband flatness, compared with a single ring case [140]. Recently, a ring response shaping technique was demonstrated via a double-injection circuit design [141,142]. By tuning coupling coefficients between the bus waveguides and the ring resonator, the FSR of the filter response can be flexibly altered from one FSR to 2 times FSR. Attractively, this photonic double-injection photonic circuit forms the basis of the recent demonstration of an on-chip photonic all-pass filter with great potential for MWP applications [143].

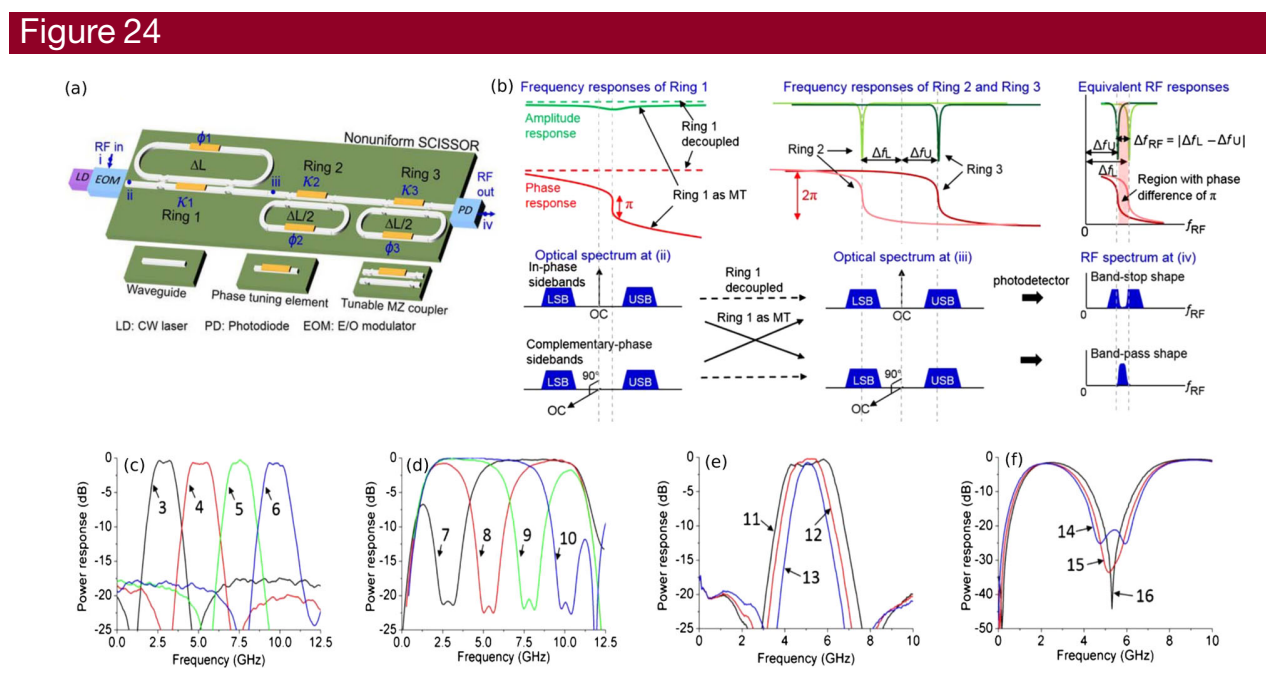

IMWP filters based on side-coupled integrated spaced sequence of resonators (SCISSORs). (a) The schematic of layout of a $\mathrm{Si}_{3} \mathrm{~N}_{4}$-based SCISSORs. (b) IMWP filter implementations using SCISSORs, showing modulation transformation and filter response formation. Measured responses of (c) passband frequency tuning, (d) stopband frequency tuning, (e) passband bandwidth tuning, and (f) stopband rejection tuning. Reprinted with permission from [70]. Copyright 2016 Optical Society of America. 


\section{1b. Subwavelength Periodic Structures}

On-chip subwavelength gratings have recently gained great interest for IMWP filters due to device compactness and simple principle [31,144-146], based on the well-established MWP applications using fiber-based Bragg gratings [91,147]. A comprehensive review of IMWP applications using chip-based subwavelength gratings can be found in Ref. [148]. A representative example of on-chip silicon subwavelength gratings is shown in Fig. 26(a). In order to reduce propagation loss and improve the grating spectral resolution, 70-nm-shallow-etched silicon ridge waveguides with a width of $1 \mu \mathrm{m}$ were employed [31], yielding a FWHM bandwidth of $0.43 \mathrm{~nm}(\sim 50 \mathrm{GHz}$ in frequency in C band), as shown in Fig. 26(b). In order to achieve a higher spectral resolution and flexible tunability for RF signal processing, on-chip phase-shifted gratings that can produce a sharp spectral response provide a feasible solution [144], as shown in Fig. 26(c). The on-chip phase shift grating was able to provide an enhanced spectral resolution of $\sim 6 \mathrm{GHz}$ with spectral programmability, as shown in Figs. 26(c) and 26(d). To further improve the spectral resolution, on-chip gratings using longer lengths $(>1 \mathrm{~mm})$ [149] and low-loss materials such as $\mathrm{Si}_{3} \mathrm{~N}_{4}[150,151]$ have been recently demonstrated.

\section{Figure 25}
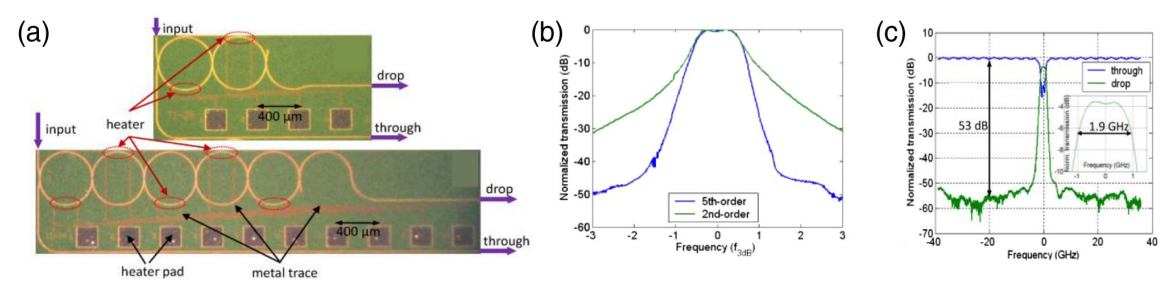

(d)

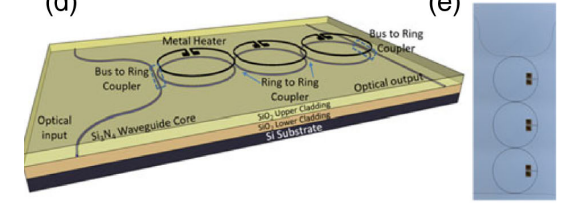

(h)

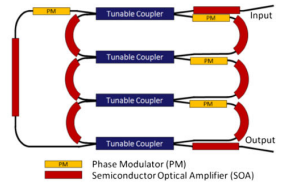

(i)

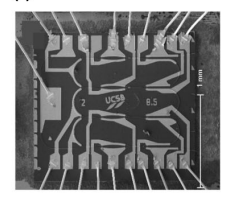

(f)

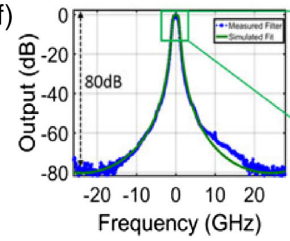

(j)

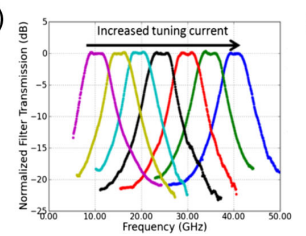

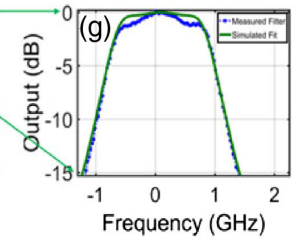

(k)

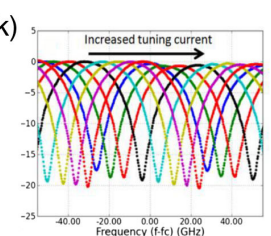

IMWP filters based on coupled-resonator optical waveguides (CROWs). (a) Optical images of a second-order and a fifth-order silicon CROWs. Reprinted with permission from [137]. Copyright 2010 Optical Society of America. (b) The comparison of normalized optical spectra of the second-order and the fifth-order CROWs at the drop ports. (c) Measured transmission spectra of the fifth-order CROWs at the through port and the drop port, respectively. (d) The schematic and (e) the optical image of the $\mathrm{Si}_{3} \mathrm{~N}_{4}$ CROWs, with (f) measured optical transmission and (g) the zoomed-in spectral of the filter passband. (C) 2018 IEEE. Reprinted, with permission, from Huffman et al., J. Sel. Top. Quantum Electron. 24, 5900209 (2018) [138]. (h) The schematic and (i) the optical image of InP-InGaAsP-based CROWs, with measured (j) bandpass responses and $(\mathrm{k})$ bandstop responses. Reprinted with permission from [56]. Copyright 2011 Optical Society of America. 


\section{1c. Ring-Assisted Mach-Zehnder Interferometer}

A more complex on-chip photonic filter scheme is the ring-assisted Mach-Zehnder Interferometer (RAMZI) that embeds ring resonator(s) in an imbalanced MZI architecture, which was initially proposed to implement rectangular-shaped spectral response for signal spectrum slicing for telecommunication applications [152].

The underlying idea of RAMZI configuration is using the steeper phase and amplitude response of ring resonators to sharpen the roll-offs of a conventional MZI. As shown in Fig. 27(a), the spectral responses of a RAMZI structure can be engineered to approximate an rectangular-shape response using multiple ring resonators $[49,153,155]$. Such a rectangular-shape filter response is unachievable for a single ring resonator except using a coupled resonator bank [56,133-135,137,138]. It should be noted that the rings' FSRs should be designed to be half of that of the imbalanced MZI. Using low-loss $\mathrm{Si}_{3} \mathrm{~N}_{4}$ waveguides, RAMZI-based filters were able to provide bandstop and bandpass filter responses with GHz-level spectral resolution and sub-GHz roll-offs. Empowered by on-chip thermal tuning elements, RAMZI's response can be reconfigured and tuned to implement more complex filter shapes [153], as shown in Figs. 27(b) and 27(c). Recently, a periodic rectangular RAMZI filter with an FSR of $25 \mathrm{GHz}$ over $4 \mathrm{THz}$ frequency range was demonstrated, as shown in Figs. 27(d) and 27(e). Such unique filters promised powerful applications for RF signal channelization and RF photonic modulation transformation [132,155,156].

Similar to the multistage configuration in digital filter design [90], cascaded filter units are able to provide for flexible and complex filter responses [32,55,157]. Unlike

\section{Figure 26}
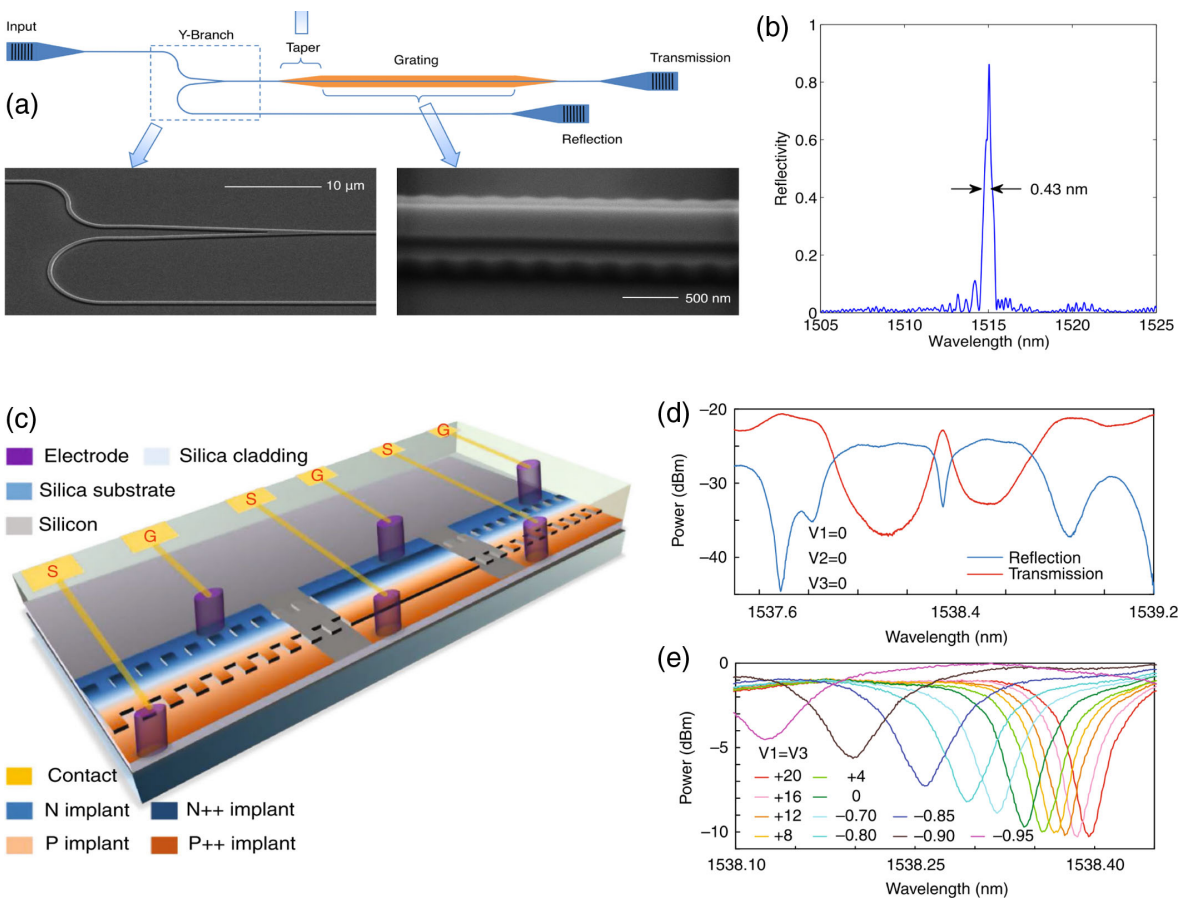

On-chip subwavelength gratings for IMWP applications. (a) Schematic of a passive silicon grating circuit layout and SEM images of key components. (b) Optical spectrum at the reflection port. Reprinted with permission from [31]. Copyright 2012 Optical Society of America. (c) Schematic of a tunable on-chip silicon subwavelength grating, (d) optical reflection and transmission spectra, and (e) zoomed-in transmission spectrum with a spectral resolution of $\sim 6 \mathrm{GHz}$. Reprinted with permission from [144]. Copyright 2019 Optical Society of America; licensed under a Creative Commons Attribution 4.0 International license. 
the mutually coupled ring resonators $[56,133-135,137,138]$, the variation in a single RAMZI unit will not affect other RAMZI units, so that the multistage RAMZI can be much less sensitive to environmental perturbations. To make the multistage RAMZI filter more compact, the dual outputs of a RAMZI can be connected to form a Sagnac loop that serves as a mirror, as shown in Figs. 28(a) and 28(b). Therefore, this novel configuration is equivalent to a two-stage cascaded RAMZI filter, providing a comb-like filter response with a much higher spectral resolution of $0.6 \mathrm{GHz}$, as shown in Figs. 28(c) and 28(d). This is in contrast to the GHz-level bandwidth in a single RAMZI filter. By altering the number of rings, the filter bandwidth can be tuned, while the filter center filter frequency can be varied by tuning the phase shifts in the resonators, as shown in Figs. 28(e) and 28(f), respectively.

\subsection{Photonic Devices Based on Nonlinear Optical Effects}

By leveraging strong light-matter interactions in chip-scale photonic circuits, on-chip nonlinear optical processes have become a powerful tool to realize advanced IMWP

\section{Figure 27}
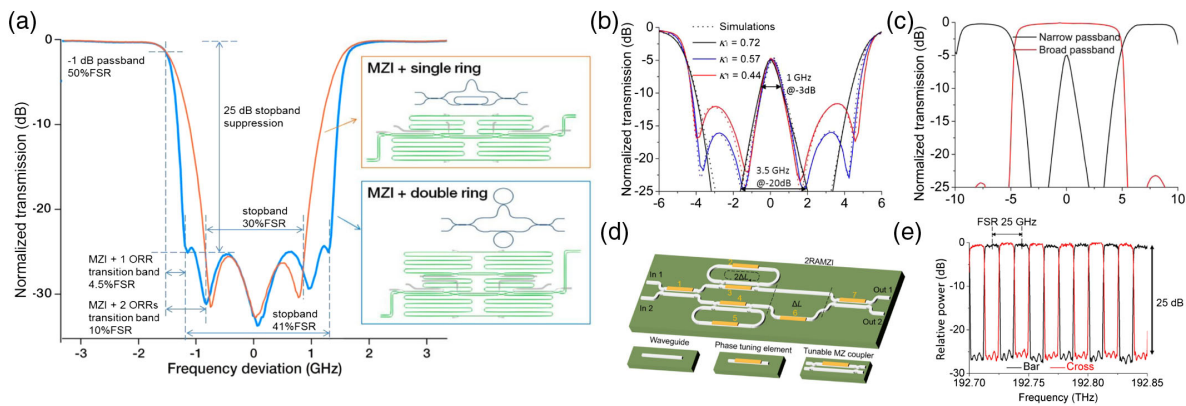

Ring-assisted Mach-Zehnder interferometer (RAMZI) filters. (a) Measured filter shapes of the single and double RAMZIs. Insets show the RAMZI filters architectures. (b) Simulated and (c) measured filter responses. (d) Schematic of a $\mathrm{Si}_{3} \mathrm{~N}_{4}$-based RAMZI and tuning elements. (e) Measured bar-port and cross-port responses. Reprinted with permission from [49,153]. Copyright 2011, 2016 Optical Society of America. (c) IEEE. Reprinted, with permission, from Zhuang et al., J. Lightwave Technol. 34, 1732-1738 (2016) [154].

\section{Figure 28}
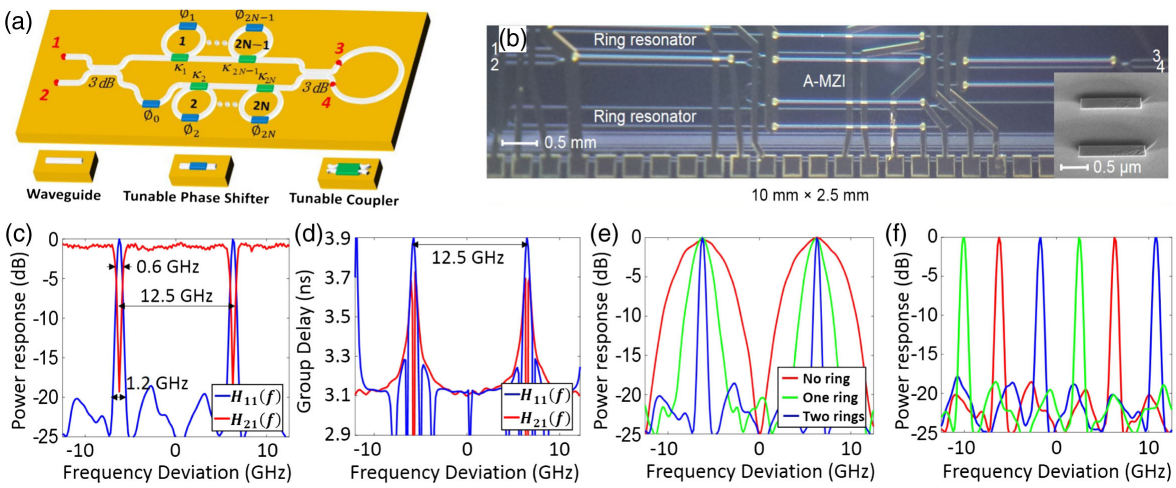

Equivalent multistage RAMZI filter. (a) The schematic of an equivalent two-stage cascaded RAMZI filters implemented by connecting two outputs of a conventional RAMZI structure. (b) Optical image of the actual device layout. Measured (c) filter responses and (d) group delays. Demonstrations of (e) bandwidth tunability and (f) center frequency tunability. Reprinted with permission from [157]. Copyright 2017 Optical Society of America. 
functionalities, which are unachievable for linear photonic devices. Through on-chip nonlinear optical processes, significantly enhanced IMWP performance has been achieved using SBS [115,158] and Kerr-effect-based optical frequency microcomb generation [159-161], achieving unprecedented high spectral resolution and an extended number of optical delay taps in ultracompact footprints, respectively. The advance and maturity of PIC technology enables the integration of linear and nonlinear photonic devices from the same or multiple materials into the same photonic circuit, allowing for the emergence of compactness, functionality, and performance.

\section{2a. On-Chip Stimulated Brillouin Scattering}

The canonical SBS describes the coherent coupling between a counter-propagating optical pump wave and probe wave, mediated by an optically induced [115,162] acoustic wave, as shown in Fig. 29(a). An intense optical pump wave at a frequency of $\omega_{p}$ is injected into the waveguide, counter-propagating with an optical wave at a lower frequency of $\omega_{s}$ (Stokes frequency). The beat note between these two optical waves can form a density variation, i.e., the acoustic wave that scatters the pump wave to Stoke frequency via the photoelastic effect. The scattered light will in turn further enhance the acoustic wave, which forms a mutually reinforced process that produces exponential amplification of Stokes light. Similarly, the anti-Stokes process enables a loss resonance at a higher frequency of $\omega_{a s}$ (anti-Stokes frequency).

The most distinct feature of the SBS process is the generation of an ultranarrow gain (loss) resonance at Stokes (anti-Stokes) frequency, with an inherent linewidth in the order of tens of $\mathrm{MHz}$ determined by the phonon wave lifetime [163,164], as shown in Fig. 29(b). Such a narrow linewidth is associated with an ultrahigh optical quality (Q) factor in the order of $1 \times 10^{7}$. Moreover, SBS response can be flexibly tuned over a broad frequency range, in contrast to conventional optical devices such as interferometer and ring resonators of which the tuning capability is limited by their periodic spectral response. The recent breakthrough in $\mathrm{As}_{2} \mathrm{~S}_{3}$ photonic circuits has enabled harnessing and inducing SBS in chip-scale devices with unprecedentedly high photonic-phononic interactions [115,158]. On-chip SBS is able to achieve significantly reduced footprint and enhanced stability [115,165-169], and can inherit the MWP functionalities and applications [170-177] established for SBS in optical fibers [109,110,178-180], in particular for integrated MWP filtering.

A typical waveguide cross section of $\mathrm{As}_{2} \mathrm{~S}_{3}$-based Brillouin waveguides [162] is shown in Fig. 30(a), which can provide a high Brillouin gain exceeding $50 \mathrm{~dB}$ $[183,184]$ with negligible nonlinear optical losses. The one-to-one spectral mapping

\section{Figure 29}
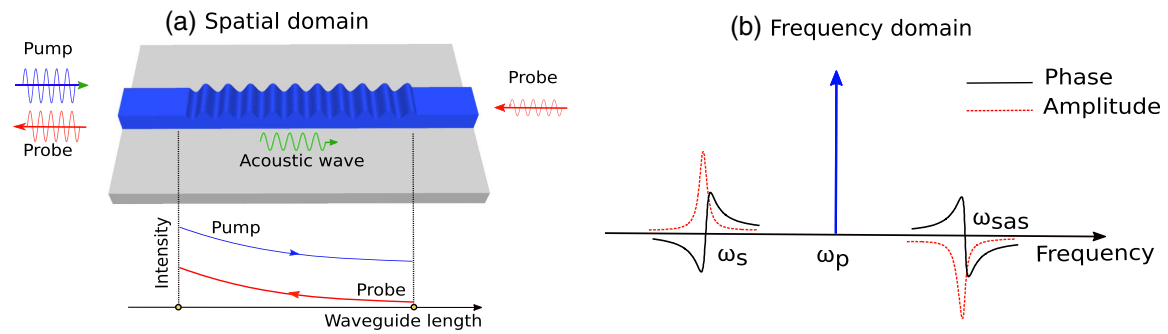

Schematic of on-chip SBS response in (a) the spatial domain and (b) the frequency domain. Under an intense CW optical pump, a very narrow optical gain resonance can be formed at Stokes frequency, with a sharp phase response over the same frequency range. At anti-Stokes frequency, a loss resonance that has a phase shift with an opposite phase slope can be induced. 
of SBS responses to the RF domain has been widely exploited to implement MWP filters [77], as well as for high-capacity optical communications [185,186]. However, it becomes extremely challenging to achieve high filter extinction, as a high filter selection requires high pump powers (hundreds of $\mathrm{mW}$ or even higher), which can easily exceed the power handling of photonic circuits. To circumvent this issue, a novel selective-RF-cancellation technique was proposed, achieving anomalously high filter extinction of $>50 \mathrm{~dB}$, while using only a few decibels of SBS gain [37]. Attractively, a spectrally broadened optical pump can produce broadband SBS responses. These optically programmable responses make it possible to utilize SBS as an efficient technology to implement IMWP filters with bandwidth tunability, without increasing structural complexity. Using the technique introduced in Subsection 2.4, a bandpass filter with tunable width from 30 to $440 \mathrm{MHz}$ was achieved using a spectrally broadened optical pump [66], as shown in Fig. 30(b). On the other hand, the filter bandwidth can also be reduced to less than $20 \mathrm{MHz}$, through sharping the Brillouin gain resonance by two adjacent Brillouin loss resonances [182,187], as shown in Fig. 30(d). A review of on-chip Brillouin-based MWP filters and applications is presented in Refs. [167,168]. Similarly, based on the RF cancellation approach in Subsection 2.4b, a bandstop filter was achieved with bandwidth tuning range up to $1 \mathrm{GHz}$, as shown in Fig. 30(c). Such a filter scheme was also implemented using a small SBS gain $(<1 \mathrm{~dB})$ in silicon [73].

\section{Figure 30}

(a)

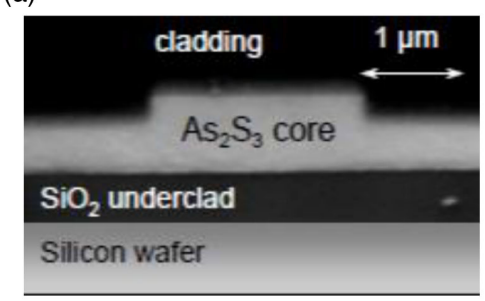

(c)

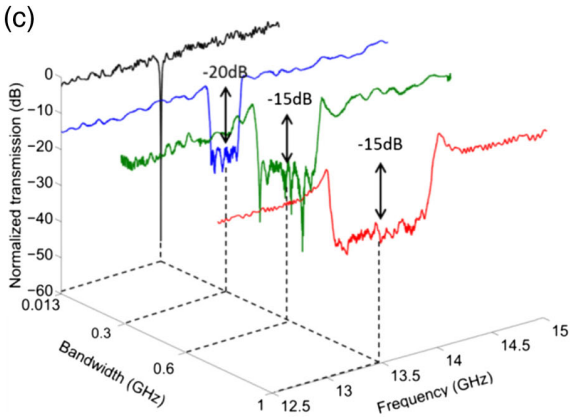

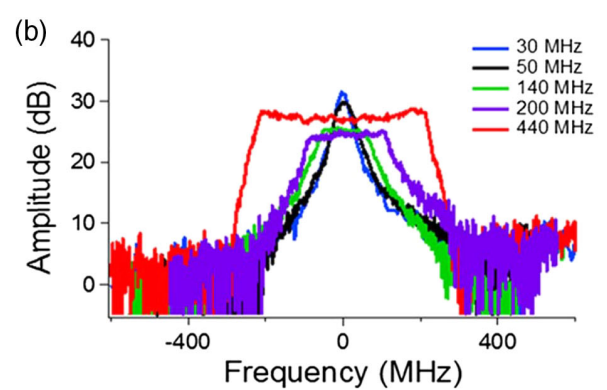

(d)

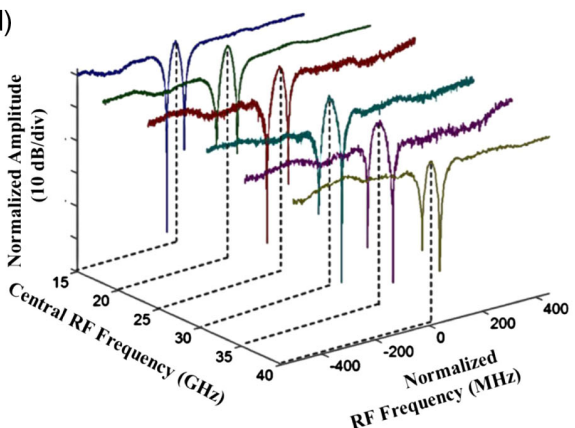

$\mathrm{As}_{2} \mathrm{~S}_{3}$-based Brillouin IMWP filters. (a) The cross section of an $\mathrm{As}_{2} \mathrm{~S}_{3}$ Brillouinactive waveguide. Reprinted with permission from [162]. Copyright 2011 Optical Society of America. (b) A bandwidth tunable IMWP bandpass filter using an onchip $\mathrm{As}_{2} \mathrm{~S}_{3}$ Brillouin circuit. A broadband passband can be achieved using SBS gain response tailoring. Reprinted with permission from [66]. Copyright 2016 Optical Society of America. (c) A Brillouin-based bandstop filter using an $\mathrm{As}_{2} \mathrm{~S}_{3}$ photonic waveguide, exhibiting a maximum bandwidth of $1 \mathrm{GHz}$. Reprinted with permission from [181]. Copyright 2016 Optical Society of America. (d) The tuning of the central frequency of the high-resolution filter enabled by the combination of Brillouin gain and loss resonances. Reprinted by permission from Macmillan Publishers Ltd.: Choudhary et al., Sci. Rep. 7, 5932 (2017) [182]. 
An integrated silicon photonic-photonic emitter-receiver (PPER) architecture [188] demonstrated a novel way to produce fine-spectrum-resolution bandpass filters [72], through harnessing on-chip SBS in suspended silicon membrane waveguides, as shown in Fig. 31(a). The key idea of a PPER-based IMWP filter is that the SBSinduced acoustic wave can transmit to an adjacent silicon waveguide and modulate the optical signal, as shown in Fig. 31(b). As a result, the modulated signal will produce optical sidebands that preserve the acoustic responses, enabling a filter spectral resolution of $5 \mathrm{MHz}$ and a filter extinction of $26 \mathrm{~dB}$, as shown in Fig. 31(c). A less obvious but very important advantage is the spatial separation of a transmitter waveguide and receiver waveguide, which can potentially minimize cross talk and noise from the optical pump signal.

To unleash the full potential of on-chip Brillouin signal processing, it is desirable to combine Brillouin-active circuits with functional linear integrated photonic devices. A high-performance IMWP reconfigurable bandstop filter based on a centimeterscale $\mathrm{As}_{2} \mathrm{~S}_{3}$ photonic circuit that pairs the Brillouin-active circuits with passive an overcoupled ring resonator was recently demonstrated [43,54], based on the principle discussed in Subsection 2.4b. This on-chip implementation enables a new class of IMWP devices that is compact and sustains all advantageous features in the earlier demonstration using SBS in a 4.6-km-long optical fiber and an integrated ring resonator [64]. Figure 32(a) shows the microscope image of the fabricated $\mathrm{As}_{2} \mathrm{~S}_{3}$ photonic chip consisting of an array of IMWP devices. Each device incorporates a ring resonator [Figs. 32(b) and 32(c)] followed by a 4-cm-long Brillouin spiral waveguide [Fig. 32(d)]. The ring resonator has a total length of $\sim 4.7 \mathrm{~mm}$, resulting in a measured FSR of around $30 \mathrm{GHz}$. The SBS spiral waveguides with a cross-section size of $1.9 \mu \mathrm{m} \times 0.68 \mu \mathrm{m}$ allowed for efficient SBS gain and low optical propagation losses, as shown in Fig. 32(e).

Figure 32(f) shows the RF notch filter responses with a filter rejection of $>40 \mathrm{~dB}$, when a single-frequency pump was used. By broadening the SBS gain response to compensate the amplitude suppression of the overcoupled ring resonator, a bandstop filter response can be implemented with a $20 \mathrm{~dB}$ bandwidth of $\sim 100 \mathrm{MHz}$ and a frequency tunability over $15 \mathrm{GHz}$, as shown in Fig. 32(g). It should be noted that the $3-\mathrm{dB}$ bandwidth of the synthesized RF notch responses is $\sim 3 \mathrm{GHz}$, which is limited by the overcoupled ring resonance. Figure $32(\mathrm{~h})$ shows the overall RF link gain in the level of $-10 \mathrm{~dB}$ in the filter passband with the noise figure ranging from $20 \mathrm{~dB}$ to $30 \mathrm{~dB}$, in the case that the notch filter response was located at $9 \mathrm{GHz}$. The current

\section{Figure 31}
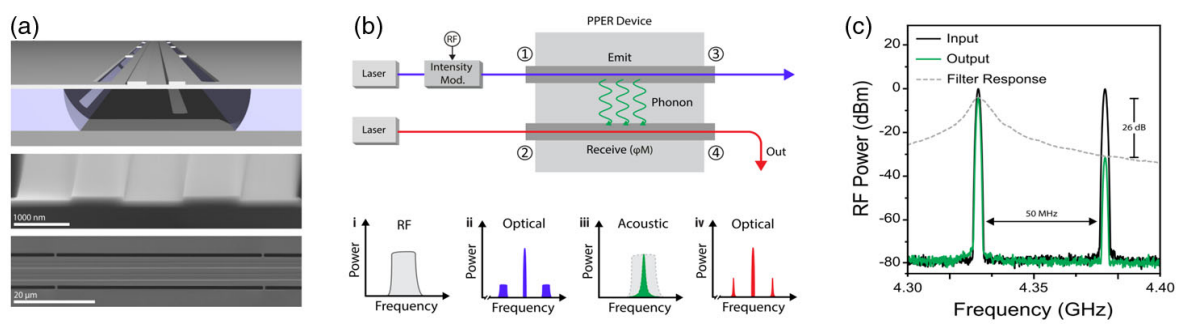

Silicon photonic-photonic emitter-receiver (PPER) Brillouin-based device. (a) The schematic and SEM images of the silicon PPER device. (b) Prince of PPER-based IMWP filter. The acoustic wave emitted from the emitter waveguide modulates the signal in the receiver waveguide, resulting in (c) a bandpass filtering response. (C) 2018 IEEE. Reprinted, with permission, from Kittlaus et al., J. Lightwave Technol. 36, 2803-2809 (2018) [72]. 
performance is limited by the relatively high insertion loss of the photonic waveguide $(18 \mathrm{~dB})$ and optical pump reflection to the photodetector. In order to further implement more complex IMWP filter functionality and advanced performance, one promising approach is using the hybrid integration technology [189-191] to integrate ultralow-loss $\mathrm{Si}$ or $\mathrm{Si}_{3} \mathrm{~N}_{4}$ ring resonators with Brillouin-active circuits, which can combine advantages of different materials.

\section{2b. Chip-Based Optical Kerr Frequency Comb}

Transverse MWP filters are in general preferably implemented in the incoherent regime to avoid mutual interference and phase fluctuations among tapped signals in photodetection using sliced broadband light sources, a laser array [95-97], or E-O frequency comb $[99,100]$. However, these light sources are bulky and can only provide a limited number of taps to achieve high filter spectral resolution. The emerging onchip optical frequency comb technology [101] provides a transformative method to achieve a compact, low-cost, and wide-spanning light source array, offering enhanced filter resolution due to the large number of taps.

A landmark demonstration of an RF photonic filter was achieved using optical frequency comb generated by an integrated $\mathrm{Si}_{3} \mathrm{~N}_{4}$ optical ring resonator via the optical Kerr effect [35]. The experimental setup of this comb-based MWP filter is shown in Fig. 33. The generated optical frequency comb [Fig. 33(b)] was truncated to provide 21 flat comb teeth [Fig. 33(c)] at the optical C band with well-tailored amplitude as an array of optical sources. After E-O modulation, the mixed combs passed through a 1.8-km-long optical fiber where the dispersion induced time delay for each tap resulted in an MWP transversal filter with a spectral resolution of $10 \mathrm{GHz}$ [Fig. 33(d)] via photodetection. Microcombs with an FSR of $200 \mathrm{GHz}$ generated using high-index doped silica glass waveguides were recently demonstrated to achieve high-performance and versatile MWP transversal filters [61]. Enabled by line-by-line programming of tap weights, various filter responses were achieved, exhibiting reduced complexity and system instability. Meanwhile, the transversal configuration also enabled the first demonstration of microcomb-based MWP true time delay lines for phased-array applications, with a high beam angular resolution. In order to achieve a finer RF filter bandwidth, more delayed taps, i.e., more comb lines, are desirable. However, the large comb FSR of typically $>200 \mathrm{GHz}$ restricts the maximum achievable number to no more than 21 within $30 \mathrm{~nm}$ wavelength range in the optical C band [35,52,192].

A chip-based MWP transversal filter that used ring resonators based on high-index doped silica glass [193] achieved a filter bandwidth tuning from 0.5 to $4.6 \mathrm{GHz}$ [44,53], as shown in Fig. 34(a). Such bandwidth reduction by more than 1 order of magnitude was achieved by generating 80 optical comb lines in $\mathrm{C}$ band, due to a reduced optical FSR of $\sim 49 \mathrm{GHz}$ using a ring radius of $\sim 592 \mu \mathrm{m}$. Figure 34(b) illustrates the experimental setup to implement the comb-based MWP filter. The Kerr optical frequency comb was generated by optically pumping the ring resonator under an intense optical power. The generated optical comb was tailored using two cascaded pulse shapers to obtain desirable weight for each tap for target RF filter shapes, as shown in Fig. 34(c). After E-O modulation and transmitting through a dispersive optical fiber, an RF filter response can be formed upon direct photodetection, achieving a minimum filter passband bandwidth of $533 \mathrm{MHz}$, as shown in Fig. 34(d). It should be noted that the tailored optical comb can be split to two arms, forming a positive tap and a negative tap for flexible RF filter response implementation. The realization of a finer filter resolution requires a dramatically increased number of taps, and thus much more microcomb lines, which is challenging for a given total bandwidth of the microcombs in the $\mathrm{C}$ band. Thus, there exists an inherent trade-off 


\section{Figure 32}
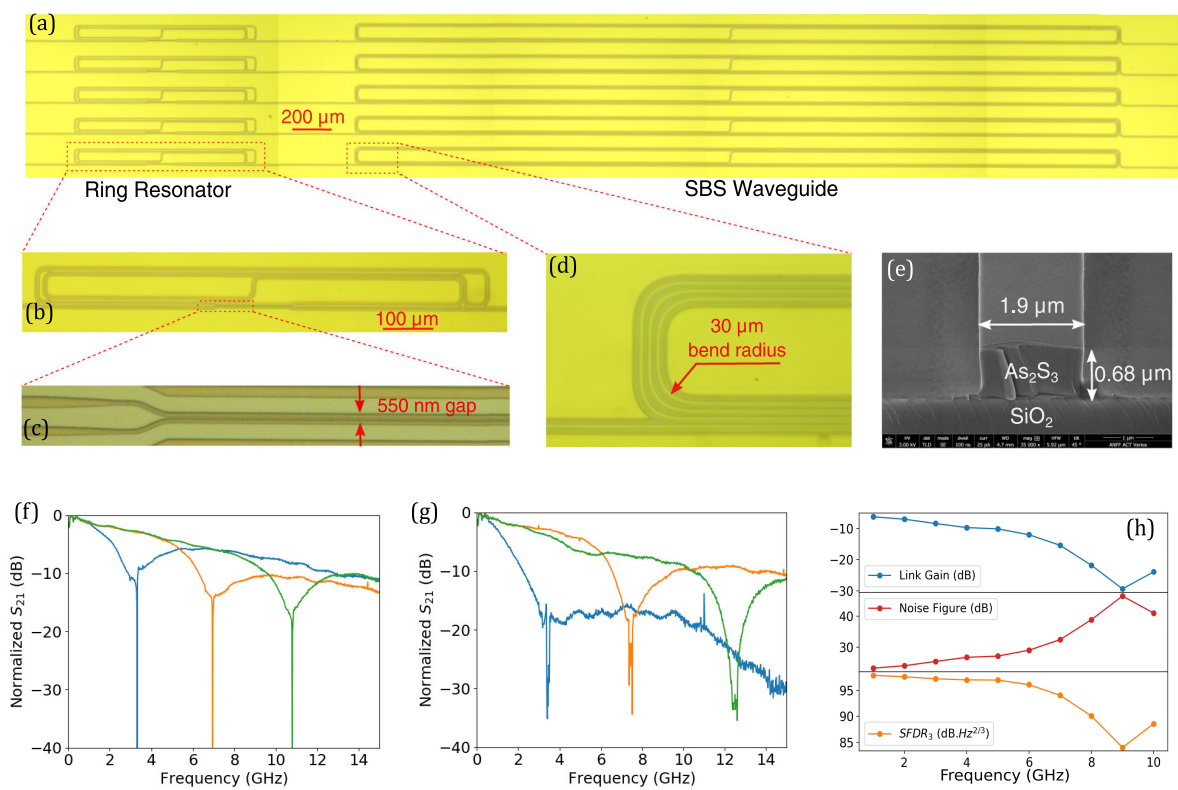

Microscope images of (a) an array of the OC ring resonator cascaded with a subsequent SBS spiral waveguides on the $\mathrm{As}_{2} \mathrm{~S}_{3}$, (b) an $\mathrm{OC}$ ring resonator, (c) the zoomed-in directional coupler with $0.85 \mu \mathrm{m}$-wide waveguides of the resonator for light coupling, and (d) the waveguide bends in the SBS spiral waveguide. (e) A scanning electron microscopy (SEM) image of the cross section of the $1.9 \mu \mathrm{m}$-wide $\mathrm{As}_{2} \mathrm{~S}_{3}$ waveguide before the silica upper cladding was sputtered. Measurement of the filter response of (f) MWP notch filters, and (g) MWP bandstop filters at different center frequencies. (h) Overall link performance of a notch filter response around $9 \mathrm{GHz}$. Reproduced from Liu et al., APL Photon. 4, 106103 (2019) [43]; licensed under a Creative Commons CC BY license.

between the filter resolution and the operation bandwidth in conventional transverse IMWP filters.

A novel microcomb-based MWP filter scheme was recently demonstrated to overcome the above limitation in multi-tap MWP filters [53], based on a new concept of RF bandwidth scaling [194]. Such a microcomb-based bandwidth-scaling filter achieved a desirable combination of high spectral resolution as a coherent filter and flexible programmability of scalable microcombs. Therefore, this novel IMWP filter scheme is able to radically decouple the filter resolution from the filter tap number. The implementation of this filter is schematically shown in Fig. 35(a), which consists of an active microring ( $\sim 49 \mathrm{GHz}$ FSR) for microcomb generation and a passive Vernier microring with a slightly different FSR for progressively spectral slicing. Each microcomb line serves as an optical carrier. A detuned optical resonance of the passive microring processes one of the sidebands of the phase-modulated optical signal, forming a bandpass RF filter response after direct photodetection based on the coherent filter scheme [depicted in Fig. 20(a)]. Thus, the achievable filter spectral resolution of $117 \mathrm{MHz}$ is determined by the fine optical resonance of the high- $Q$ passive microring. Due to the difference in ring FSRs, the RF bandpass responses from all the microcomb carriers add up in a staggered manner to form a broader bandpass RF filter response of up to $4.64 \mathrm{GHz}$ with 80 comb lines, as shown in Fig. 35(c). An additive spectrum shaper was used to achieve flexible and dynamic reconfiguration of filter responses, as shown in Fig. 35(d). By thermally tuning the relative detuning of two 
microrings' resonances, the center frequency of the synthesized RF filter response can be continuously varied from 3.3 GHz to $19.4 \mathrm{GHz}$, as shown in Fig. 35(e).

However, reported microcomb-based transversal filters relied on complicated spectral tailoring provided by bulky and expensive spectrum shapers. Therefore, the photonic integration of the spectrum shapers is highly desirable to miniaturize the microcomb-based filters. Fortunately, an integrated 32-channel line-by-line optical pulse shaper has recently been demonstrated with millisecond reconfigurability, using an InP-based arrayed waveguide grating structure for channelization and semiconductor optical amplifiers (SOAs) for precise gain tuning in each channel [106]. This impressive demonstration makes it promising to achieve more compact comb-based transversal IMWP filters, with fast reconfiguration speed. A complete novel all-optical reconfiguration of a microcomb-based transversal MWP filter was recently reported, which can radically eliminate the need for spectrum shapers [195]. This novel microcomb-based RF filter was achieved by leveraging the intrinsically rich soliton configurations including the smooth spectral soliton envelop, reconfigurable soliton crystals, and dual soliton interference. Such a high level of reconfigurable soliton properties enabled the demanding filer tunability such as passband frequency division, frequency tuning, and filter response shaping, without using any pulse shaper.

The emerging ultra-low-loss $\mathrm{LiNbO}_{3}$-on-insulator (LNOI) photonic circuits enable a promising platform to achieve a compact comb-based MWP filter. The LNOI circuits are able to simultaneously provide wideband Kerr comb generation [196,197], efficient E-O modulation [196,198,199], ultra-low-loss photonic connections [200], and high-resolution linear optical filtering [201]. These critical photonic and optoelectronic components for integrated MWP filters and systems have been simultaneously

\section{Figure 33}

(a) Kerr comb generation
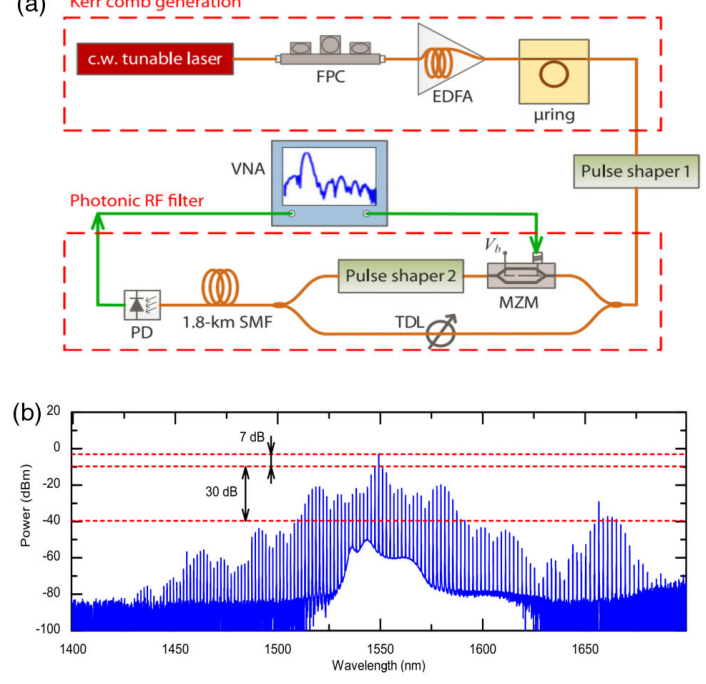
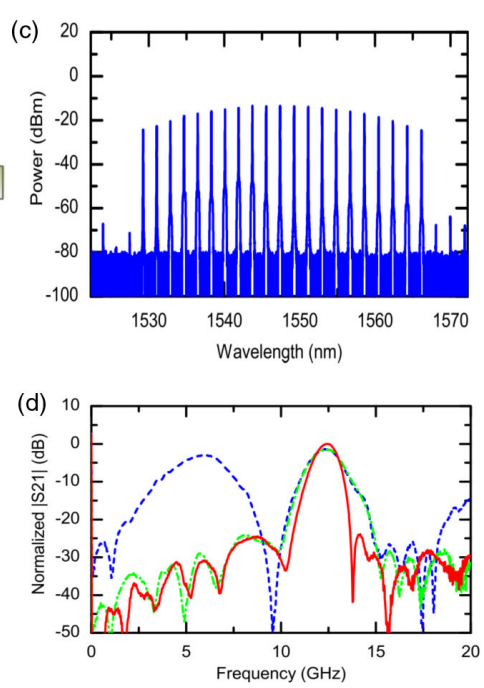

(a) Experimental setup of an MWP transversal photonic filter based on optical frequency comb generated in an integrated $\mathrm{Si}_{3} \mathrm{~N}_{4}$ photonic ring resonator. (b) Spectrum of the optical frequency comb from the microring resonator. (c) Shaped comb spectrum after the first-stage pulse shape. (d) Measured RF filter responses of a dual-passband filter, and a single-passband filter after suppressing the unwanted passband. (C) 2014 IEEE. Reprinted with permission from Xue et al., J. Lightwave Technol. 32, 3557-3565 (2014) [35]. 
integrated on the same photonic chip [197], as shown in Fig. 36. Such a high level of performance was recently proven viable to be operated using complementary metaloxide-semiconductor-compatible voltages. This significant breakthrough will lead to a new class of high-performance IMWP systems.

\subsection{State-of-the-Art Advancements for IMWP Filters}

\section{3a. Monolithic Integrated Microwave Photonic Filter}

There have been abundant efforts in realizing a higher degree of photonic integration of MWP filters, targeting the ultimate goal of fully integrated MWP filters. One of the most important aspects for achieving this goal is finding optimized material platforms that can offer all the critical optoelectronic and photonic components.

III-V-material-based integrated photonic platforms have been widely adopted in commercial products over the last half century $[9,202]$, due to the fact that III-V materials including $\mathrm{InP} / \mathrm{InGaAsP}$ have a direct and tunable bandgap that allows for bandgap engineering, electrically pumped light emission, and tunable refractive index. These attractive features enable a rich bank of on-chip devices, such as tunable lasers, SOAs, modulators, tunable devices, and high-efficiency photodetectors on a monolithic photonic chip. Early demonstrations of a tunable and reconfigurable MWP filter have been reported, through integrating SOAs and phase modulators to rapidly adjust the filter functions $[32,39,56]$. Recently, a milestone demonstration has been made to implement the first fully integrated MWP filter [40]. A coherent MWP filter scheme based on an optical-to-RF spectrum transfer was integrated on a single InP/InGaAsP photonic chip, as shown in Figs. 37(a) and 37(b). Based on such a compact photonic circuit, an RF bandpass filter with GHz-level bandwidth and moderate tunability

\section{Figure 34}

(a)

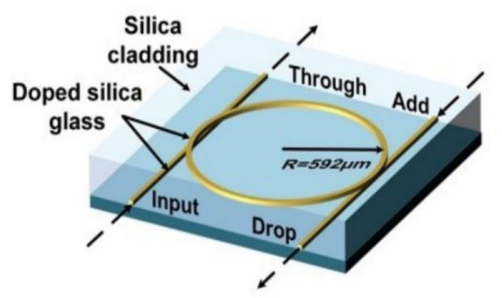

(c)

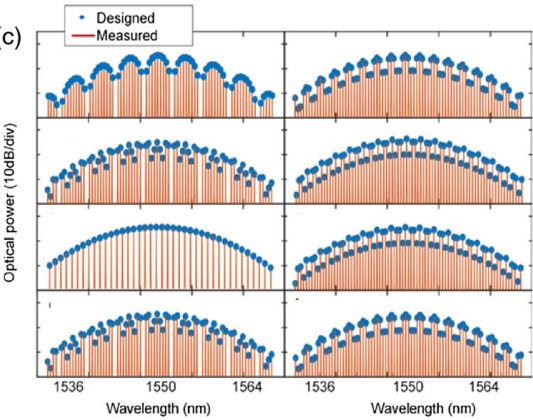

(b)

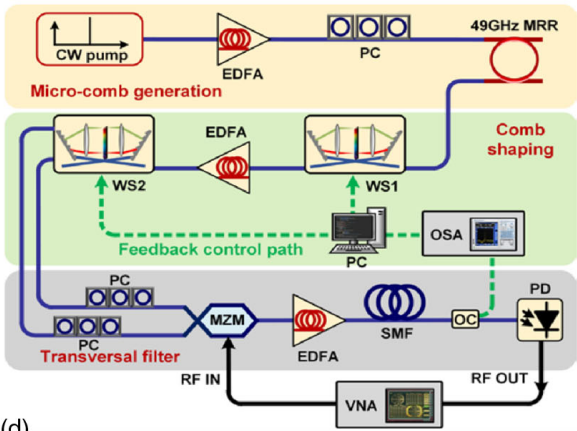

(d)

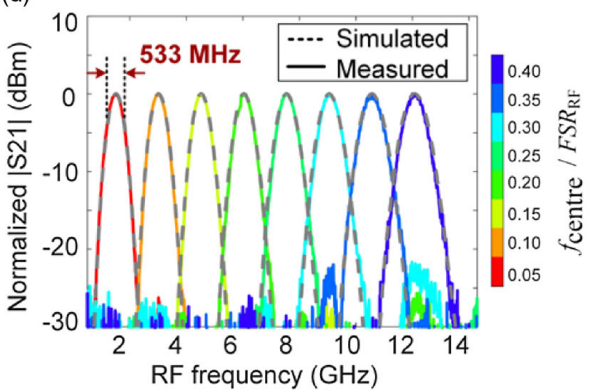

(a) Schematic illustration of the integrated microring for generating the Kerr optical microcomb using high-index doped silica glass. (b) Experimental setup for realizing the IMWP transversal filter based on $80 \mathrm{comb}$ lines. (c) Optical spectral examples of the shaped microcomb for Gaussian apodized sinc filter with various RF center frequencies. (d) Measured and simulated RF filter responses with center frequency tunability. (C) 2019 IEEE. Reprinted with permission from Xu et al., J. Lightwave Technol. 37, 1288-1295 (2019) [44]. 
was successfully demonstrated, as shown in Fig. 37(c). Although the current filter performance is constrained by the limited modulation bandwidth, relatively high waveguide losses, and RF crosstalk, this breakthrough made the first inspiring step toward fully integrated IMWP systems. Fortunately, the future performance can be

\section{Figure 35}

(a)

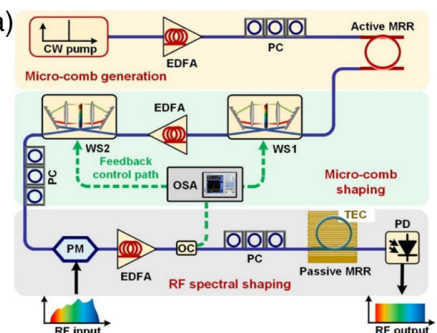

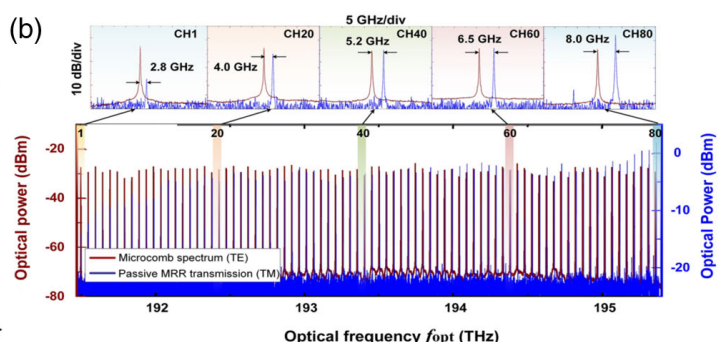

Wavelength $(\mathrm{nm})$

(c)
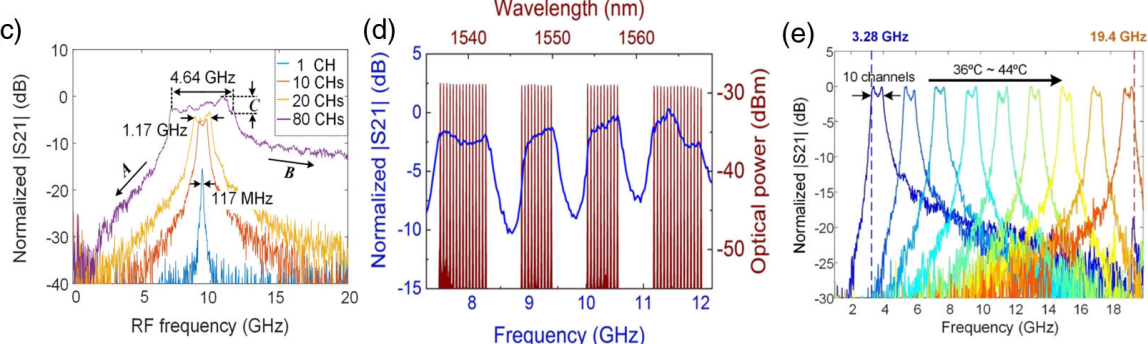

(a) Experimental implementation of the microcomb-based MWP filter using a bandwidth scaling technique. (b) Measured optical spectra of the generated microcomb and the drop-port transmission of the passive microring. Zoomed-in views show the channels with different frequency detuning. (c) Measured RF filter responses as the number of microcomb lines increased from 1 to 80. (d) Measured RF filter response featuring binary coded channel weights and the corresponding optical spectra of the shaped microcomb by spectral shapers. (e) Measured RF filter responses with varied center frequencies achieved by thermally tuning the passive microring resonance frequency. Reproduced from Xu et al., APL Photon. 4, 026102 (2019) [53]; licensed under a Creative Commons CC BY license.

\section{Figure 36}

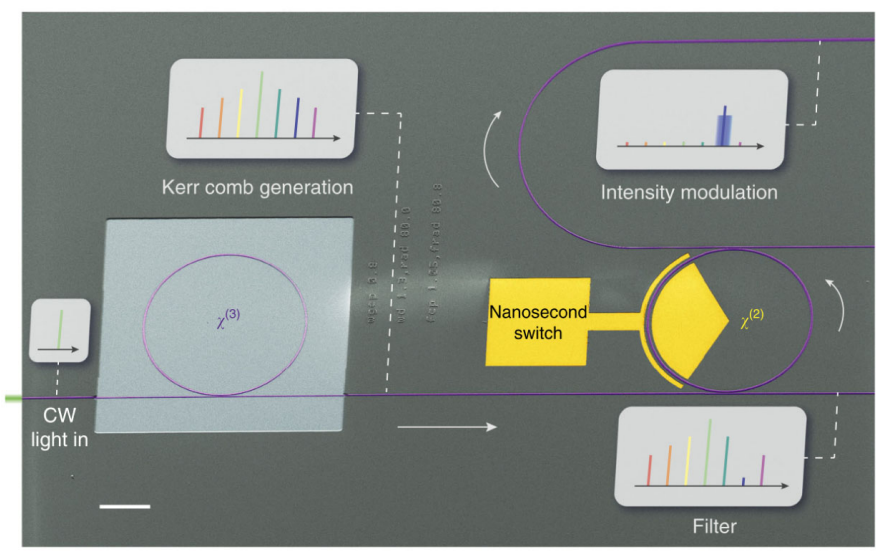

Schematic of a monolithic integrated photonic circuit that combines signal generation, modulation, and filtering, showing great potential for achieving high-performance IMWP filters. Reprinted by permission from Macmillan Publishers Ltd.: Wang et al., Nat. Commun. 10, 978 (2019) [197]. 
improved based on the promising progress of high-performance III-V-based onchip components, including $>20 \mathrm{dBm}$ laser source with a $\mathrm{RIN}<-140 \mathrm{dBc} / \mathrm{Hz}$, MZMs with a low half-wave voltage in a range of $1.5 \sim 4 \mathrm{~V}$, and high-efficiency photodetectors with a responsivity of up to $0.9 \mathrm{~A} / \mathrm{W}$ [9,202-209].

On the other hand, the silicon-based photonic circuit is a widely used platform for IMWP filters [210,211], due to its inherent advantages including compact devices size, low waveguide losses [67,126,212], and the availability of on-chip high-speed modulators [213-216] and photodetectors [217-220]. Based on these advantages, a silicon-based IMWP bandpass filter was recently reported [221], using an on-chip microdisk resonator for sideband filtering, as shown in Fig. 38(a). A filter with 3-dB bandwidth of $\sim 2 \mathrm{GHz}$ and frequency tunability less than $10 \mathrm{GHz}$ was achieved. This implementation of an IMWP filter with most key components integrated on a chip marks an encouraging step forward. However, achieving coherent and electrically pumped light sources on silicon is extremely challenging and is still missing, due to its indirect band structure. To bypass this issue, combing two well-explored and mature III-V and silicon-based platforms [222-224] provides an efficient solution. The III-V/Si heterogeneous integration can be achieved by flip bonding the III-V laser dies on the silicon circuits [223,225-228]. A novel III $-\mathrm{V} / \mathrm{Si} / \mathrm{Si}_{3} \mathrm{~N}_{4}$ laser was recently demonstrated to enable an electrically pumped laser for the first time, allowing for the multilayer heterogeneous photonic integration of high-performance lasers and ultralow-loss functional $\mathrm{Si}_{3} \mathrm{~N}_{4}$ circuits [229]. With these complete functional components, the III-V/Si platform [230] and the III $-\mathrm{V} / \mathrm{Si} / \mathrm{Si}_{3} \mathrm{~N}_{4}$ [229] platform will have great potential to enable high-performance fully integrated IMWP filters and systems.

\section{3b. Programmable and Multifunctional MWP Circuits}

Over the last decade, most demonstrations of the IMWP filters were achieved by using application-specific integrated photonic circuits (ASPICs) with limited tunability and reconfigurability, targeting specified filter responses. For software-defined RF applications, it is quite attractive if an integrated photonic circuit can be flexibly and dynamically reconfigured to realize multiple functionalities.

In contrast to the ASPICs approach, a remarkable concept for generic programmable architecture based on a two-dimensional (2D) waveguide mesh network was recently proposed and demonstrated [38], featuring improved flexibility in arbitrary function programmability and reconfigurability for photonic and MWP signal processing. The

\section{Figure 37}

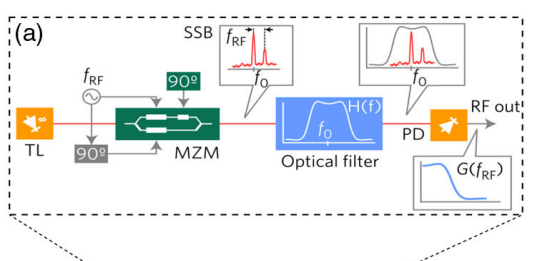

(b)
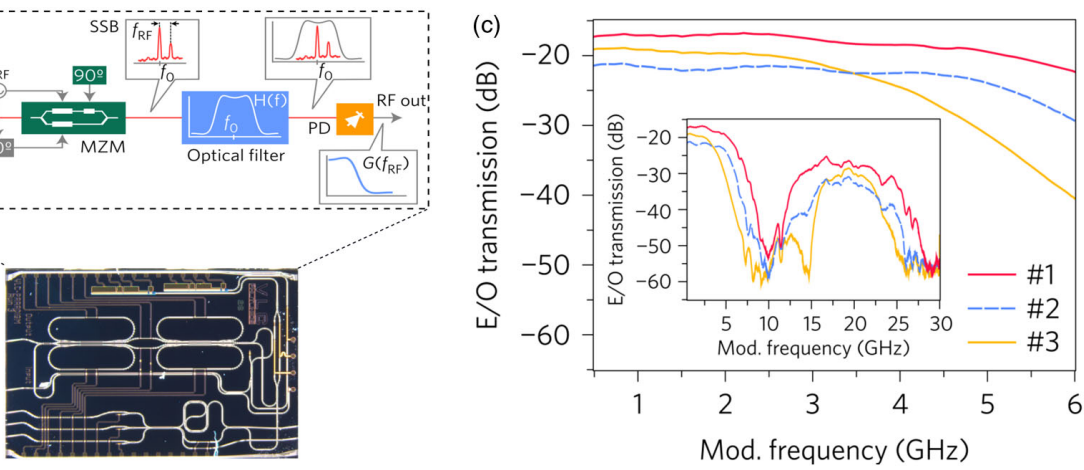

Fully integrated MWP filter. (a) The MWP filter scheme and (b) the image of monolithically integrated photonic chip. (c) Demonstrated RF bandpass filter response. Reprinted from Fandiño et al., Nat. Photonics 11, 124-129 (2017) [40]; licensed under a Creative Commons CC BY license. 
2D mesh network concept was first demonstrated in a square-shaped mesh topology using a $\mathrm{Si}_{3} \mathrm{~N}_{4}$ photonic chip, as shown in Fig. 39(a). The core design concept of this universal processor is to emulate the hardware design for a field-programmable gate array (FPGA), which is ubiquitously used in electronics, using a large network of identical photonic cells that consist of tunable MZIs and phase shifters. In such a novel design, each Mach-Zehnder coupler serves as a basic programmable unit, which can be configured as a $2 \times 2$ coupler or a simple-pass waveguide for optical signal routing. With two mesh units, this level of flexible reconfigurability allowed for the implementation of optical filter structures and responses, as shown in Fig. 39(b). With a large scale of mesh work, multiport switch and routing network can be realized for complex functionalities [231-233].

A hexagonal-shaped mesh topology with seven programmable units [42] was subsequently reported using silicon photonic circuits, as shown in Figs. 40(a) and 40(b). Each unit allows for independent control of both the amplitude and phase, resulting in the implementation of numerous software-defined functions [234]. This hexagonal lattice network is able to offer several advantages including enhanced reconfiguration performance, more switching elements per unit area, and lower losses per spatial resolution, compared with the square mesh network, which was investigated in an earlier theoretical study [235]. With increased programmable units and improved

\section{Figure 38}
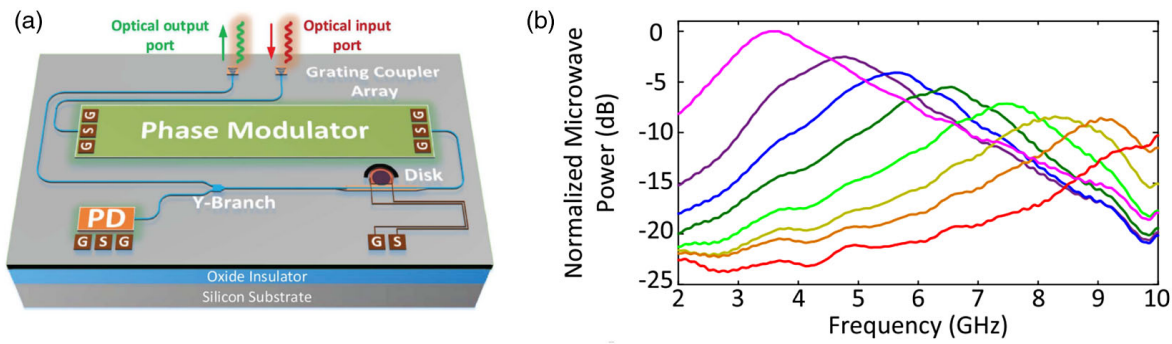

Silicon-based IMWP filter. (a) Schematic of the proposed integrated MPF on a silicon photonic chip. (b) Measured RF filter responses with the center frequency tuned from 3 to $10 \mathrm{GHz}$. (C) 2015 IEEE. Reprinted with permission from [221]. Copyright 2018 Optical Society of America.

Figure 39

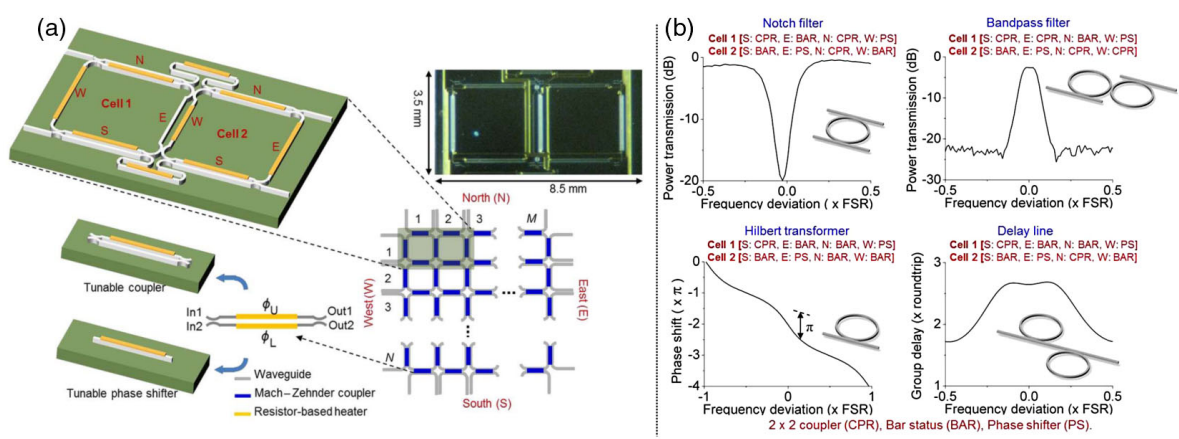

(a) Schematic and an image of the square mesh programmable network and the key components. (b) Four optical filter configurations by programming the circuit, with the measurements of the corresponding filter response. Reprinted with permission from [38]. Copyright 2015 Optical Society of America. 


\section{Figure 40}

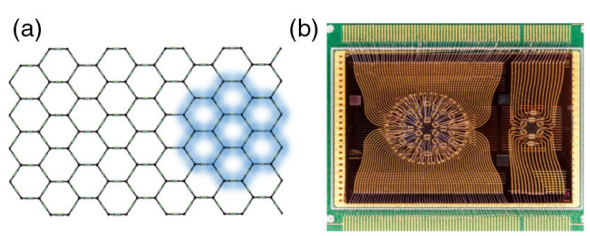

(d)

(c)
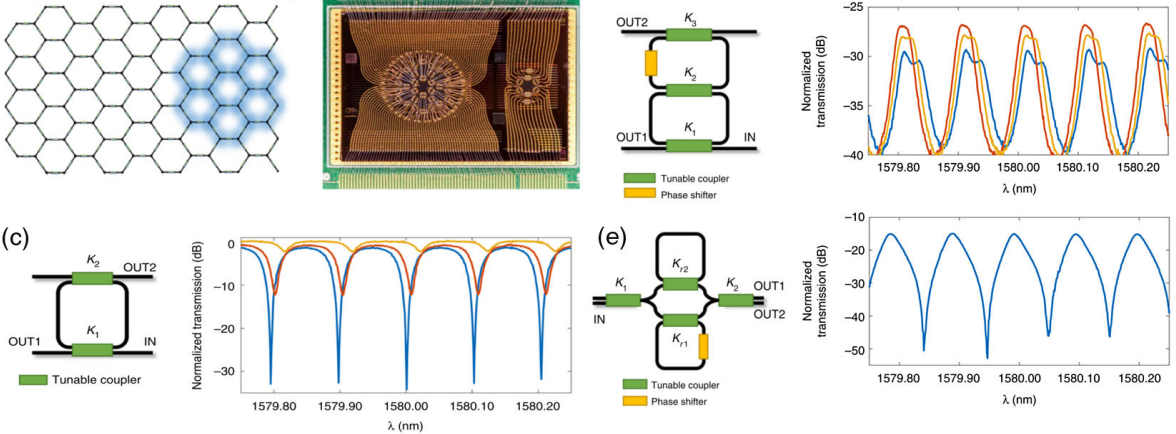

(a) Schematic of the hexagonal waveguide mesh. (b) Fabricated silicon-based hexagonal waveguide mesh with a footprint size of $15 \times 20 \mathrm{~mm}$. Scale bar is $2 \mathrm{~mm}$. Programmed filter layout and the corresponding filter responses for (c) an add-drop ring, (d) coupled-resonator optical waveguide (CROW), and (e) a ring-assisted Mach-Zehnder interferometer filter. Reprinted from Pérez et al., Nat. Commun. 8, 636 (2017) [42]; licensed under a Creative Commons Attribution 4.0 International License.

performance, this hexagonal mesh network provided 21 signal processing functionalities [236]. Representative filter responses based on an add-drop ring, CROWs, and a RAMZI configurations are shown in Figs. 40(c)-40(e), respectively.

Multifunctional MWP devices can also be achieved by operating the on-chip laser sources in a different status and selecting different components via optical switching [237]. As shown in Figs. 41(a) and 41(b), a recent demonstration of a multifunctional integrated photonic circuit that consists of four laser diodes and two modulators achieved various MWP functionalities including microwave signal generation, transmission, and filtering. The key idea is operating the on-chip distributed Bragg reflection lasers as light sources and optical amplifiers to flexibly assemble various MWP filter architectures, as shown in Figs. 41(c) and 41(d), respectively. A similar reconfigurable cell was also demonstrated to achieve programmable IMWP filters in InP platform [32] and a reconfigurable photonic signal processor [39].

\section{3c. Functionality Features of State-of-the-Art Chip-Based MWP Filters}

Figure 42 provides a summary of functionality features of state-of-the-art chipbased MWP filters, highlighting important metrics of high filter bandwidth and filter extinction. On-chip optical resonators including microrings and microdisks are the most widely used photonic devices for implementing chip-based MWP filers; their achieved spectral resolutions have been greatly improved to sub-GHz level due to the recent advancements in ultralow-loss waveguide technologies [49,51,114,124-128]. The on-chip SBS process is able to provide inherently narrowband spectral resolution of the order of tens of $\mathrm{MHz}[37,72,73,77]$, achieving comparable resolutions with conventional RF filters. Recent progress on optical microcombs has enabled subGHz-level filter bandwidth using 80 delayed taps [44]. The novel synergy of linear and nonlinear optics opens a new way to achieve tunable and high-resolution bandwidth $[43,54,64]$. On the other hand, the new class of RF-interference-based MWP filter technique allows for achieving filter out-of-band rejection more than $50 \mathrm{~dB}$ $[37,41,64,66,67,76]$, compared with conventional MWP filters based on spectrum mapping and tapped delay lines. According to the summary presented in Fig. 42, the optimal combination of RF-interference technique and high-resolution photonic filtering is able to achieve unparalleled IMWP filtering functionalities. 


\section{Figure 41}
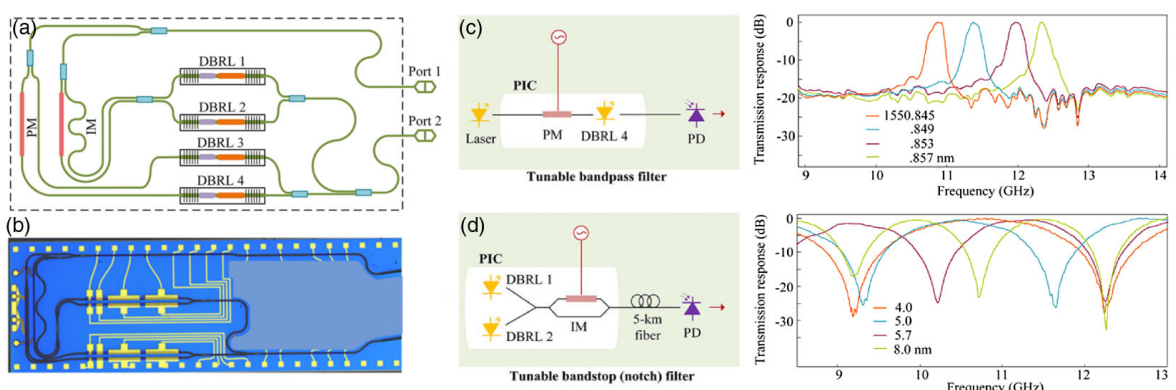

Multifunctional InP-based photonic integrated circuit. (a) Circuit layout and (b) the optical image of the fabricated photonic circuit. (c) Filter configurations and measured RF filter responses for (c) a bandpass filter and (d) a bandstop (notch) filter. Zou et al., Laser Photon. Rev. 13, 1800240 (2019) [237]. Copyright Wiley-VCH Verlag $\mathrm{GmbH} \& \mathrm{Co}$. KGaA. Reproduced with permission

\section{Figure 42}

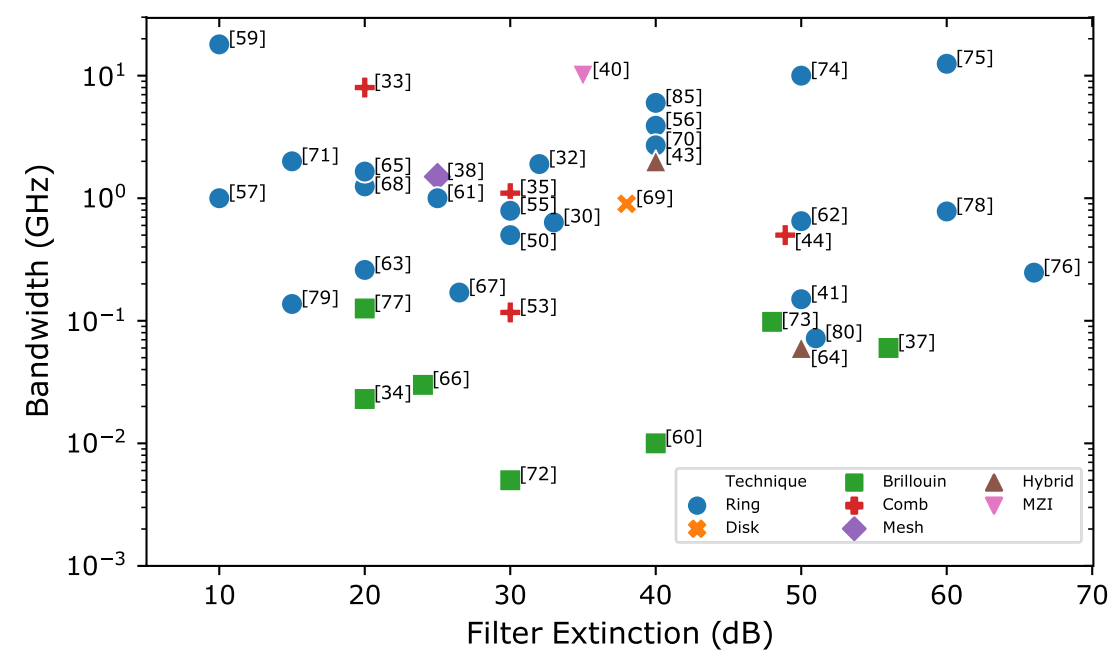

Summary of spectral resolution and filter extinction of state-of-the-art IMWP filters. Surveyed data for IMWP filters from [30,32-35,37,38,40,41,43,44,50,53,55-80].

\section{IMWP FILTER RF PERFORMANCE}

The key metrics to examine the RF performance for MWP filters are link gain, noise figure, and SFDR, which are the same measures for pure analog RF photonic links. Here, we consider an IMWP filter represented by a two-port black box which interfaces with an RF input and an output, as shown in Fig. 43. In order to have an intuitive understanding of these metrics, we start with examining the schematic diagram that qualitatively shows the signal deteriorations. An RF signal input consisting of two RF tones $\left(\omega_{1}\right.$ and $\left.\omega_{2}\right)$ separated by a frequency spacing of $\omega_{2}-\omega_{1}$ are used as the input, with a very low background noise only from the thermal noise, as shown in Fig. 43. After passing through the IMWP filter passband, the output RF signal spectrum exhibits attenuation, increased background noises, and new frequency components. These effects will degrade the signal SNR and generate new frequencies. We now present the basic equations for evaluating the link performance metrics. 


\subsection{Key RF Performance Metrics}

\section{1a. RF Link Gain of Filter Passbands}

For an IMWP filter with a two-port network configuration, one of the most important measures is the frequency-dependent transmission coefficient $S_{21}(\omega)$ that describes the system transmission property. The RF link gain at RF frequency $\omega$ of an MWP filter is defined as the ratio of the average output power $P_{\mathrm{RF}, \text { out }}$ to the average input power $P_{\mathrm{RF}, \text { in }}$, given by

$$
G(\omega)=\left|S_{21}(\omega)\right|^{2}=\frac{P_{\mathrm{RF}, \text { out }}}{P_{\mathrm{RF}, \text { in }}} .
$$

The link gain is usually expressed in decibels, given by

$$
G_{\text {link }}(\omega)=10 \log _{10} G(\omega) \text {. }
$$

One of the main sources of RF link gain degradation in MWP systems is the inefficient O-E/E-O conversion, which usually results in a very high RF loss [1]. The RF link gain is very sensitive to the optical losses, due to the square-law photodetection. In other words, an optical loss of $1 \mathrm{~dB}$ will be translated into an RF loss of $2 \mathrm{~dB}$. Hence, in the design of the MWP link and systems, any excessive optical losses should be avoided or minimized; this forms the underlying motivation of using lowloss integrated photonic circuits for high-performance IMWP systems, such as $\mathrm{Si}_{3} \mathrm{~N}_{4}$ photonic circuits $[51,114,128]$.

To offset these optical losses, a high-power light source is preferable to lift the transmitted optical power and therefore increase the photocurrent. However, it raises the requirement of a high-power-handling modulator and photodetector. An alternative solution is to use optical amplifiers, but it can introduce detrimental amplification noise and a high power level would still require a high-power-handling photodetector.

\section{1b. Noise Figure}

The noise performance is evaluated by the noise factor, which indicates the ratio of the $\mathrm{SNR}$ at the input $\left(\mathrm{SNR}_{\text {in }}\right)$ to that at the output $\left(\mathrm{SNR}_{\text {out }}\right)$. The noise factor is usually expressed in decibels, i.e., the noise figure, given by

\section{Figure 43}

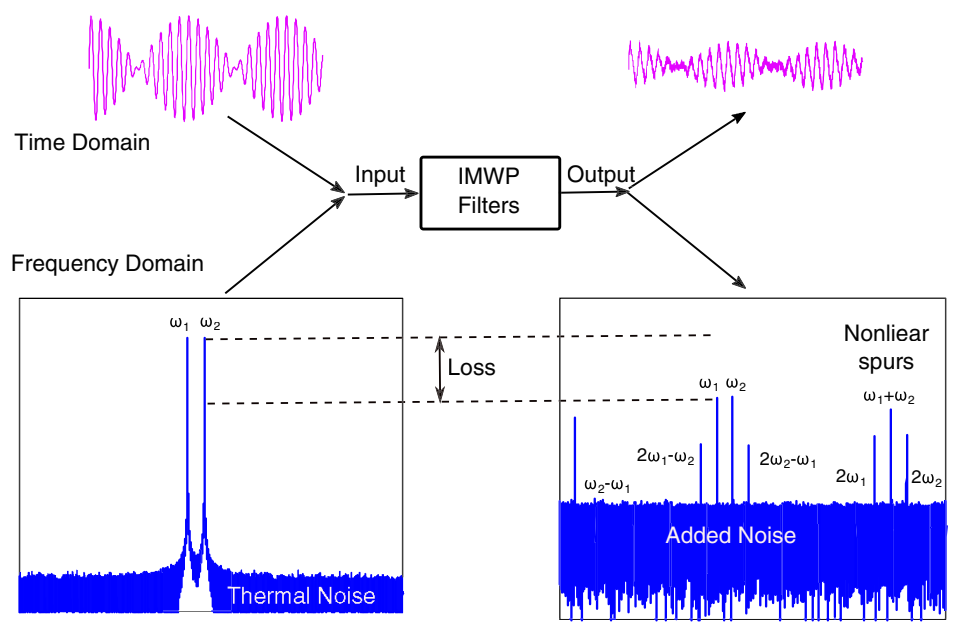

Schematic of the RF signal degradations after passing through the passband of an IMWP filter in the time domain and the frequency domain, respectively. Signal degradations typically include attenuation, harmonic spurs, and increased noise. 


$$
\mathrm{NF}=10 \log _{10}\left(\frac{\mathrm{SNR}_{\mathrm{in}, \mathrm{th}}}{\mathrm{SNR}_{\mathrm{out}}}\right),
$$

where $\mathrm{SNR}_{\mathrm{in}, \text { th }}=P_{\mathrm{RF}, \text { in }} / P_{N, \text { th }}$ is the input SNR with an assumption that only the thermal noise exits at the input, and $\mathrm{SNR}_{\text {out }}=P_{\mathrm{RF} \text {,out }} / P_{N}$ is the output SNR with a total noise power $P_{N}$. The thermal noise is caused by the thermal fluctuations of the charge carriers in resistors. The thermal noise power $P_{N, \text { th }}$ over the bandwidth $B$ at temperature $T$ is given by

$$
P_{N, \text { th }}=k_{B} \mathrm{~TB},
$$

where $k_{B}$ is Boltzmann's constant. At the standard noise temperature $T_{s}=290 \mathrm{~K}$, the thermal noise power in decibels at $1 \mathrm{~Hz}$ bandwidth is $-174 \mathrm{dBm} / \mathrm{Hz}$, which is usually termed as the thermal noise power spectral density (PSD). By substituting Eqs. (19) and (22) into Eq. (21), the noise figure is then given by

$$
\mathrm{NF}=10 \log _{10}\left(\frac{P_{N}}{\mathrm{G}_{\text {link }} k_{B} \mathrm{~TB}}\right)
$$

The noise figure in Eq. (23) is usually written in decibels for simplicity, given by

$$
\mathrm{NF}(\mathrm{dB})=P_{N}(\mathrm{dBm} / \mathrm{Hz})-\mathrm{G}_{\text {link }}+174(\mathrm{dBm} / \mathrm{Hz}) .
$$

In general, the total noise power delivered to the RF signal consists of different types of noises, given by

$$
P_{N}=P_{N, \text { shot }}+P_{N, \text { RIN }}+\left(1+\mathrm{G}_{\mathrm{link}}\right) P_{N, \text { th }}+P_{N, \text { amp }},
$$

where $P_{N \text {,shot }}$ is the shot noise, $P_{N, \text { RIN }}$ is the relative-intensity noise (RIN), and the $P_{N \text {,amp }}$ is the amplification noise induced by the RF or optical amplifiers used in the MWP systems in some cases. The amplifier noise $P_{N \text {,amp }}$ usually cannot be written in a closed form, but it can be measured indirectly. The shot noise is caused by the photocurrent fluctuations due to the random arrival of received photons [238], expressed as

$$
P_{N, \text { shot }}=2 q I_{\text {det }} B R_{\text {load }},
$$

where $q$ is the elementary charge constant and $I_{\text {det }}$ is the detected photocurrent. The RIN noise originates from the random fluctuations of the laser output intensity usually caused by the spontaneous emission of the gain medium in a laser cavity, pump intensity fluctuations, and the instability of the laser cavity [238]. The detected RIN noise is written as

$$
P_{N, \mathrm{RIN}}=10^{\frac{\mathrm{RIN}}{10}} I_{\mathrm{det}}^{2} B R_{\mathrm{load}},
$$

where $\mathrm{RIN}(\mathrm{dB})=10 \log _{10}(\mathrm{rin})$ is the laser RIN PSD in decibels, which is frequencydependent. Therefore, the RIN noise depends both on the laser property RIN and the square of the detector photocurrent.

Figure 44 shows the calculated noise PSDs of the RIN noise, the shot noise, thermal noise, and the total noise, respectively, when the received optical power is varied in a photodetector with a representative responsivity of $0.5 \mathrm{~A} / \mathrm{W}$ and load impedance. When the received optical power increases, the shot noise increases linearly as a function of the received optical power. In contrast, the RIN noise varies quadratically as a function of the received optical power. At a high photocurrent, the RIN noise will be the dominant noise source over other noise (RIN-noise-limited regime). 
From Eq. (24), one can clearly find that the noise figure of an MWP filter depends both on the total noise power [Eq. (25)] and on the link gain [Eq. (20)]. Hence, to achieve a low noise figure, important efforts need be made to increase the optical power in the MWP link and minimize the noise at the same time. However, there exists a trade-off; increasing the optical power will increase the total noise power that is associated with the laser power, i.e., the photocurrent. In the next sections, we will review the recent progress on a chip-based MWP filter with high RF link gain and low noise figure.

\section{1c. Spurious-Free Dynamic Range}

SFDR is used to quantify the signal distortions induced by the system response nonlinearity. The system nonlinear responses to the input RF signal will give rise to the generation of new frequencies, as schematically shown by the spurs in Fig. 43. The spurs mainly include the second-order harmonic distortions (HD2) at the frequencies $2 \omega_{1}$ and $2 \omega_{2}$, the second-order intermodulation distortions (IMD2) at the frequencies $\omega_{1}-\omega_{2}$ and $\omega_{1}+\omega_{2}$, and the third-order intermodulation distortions (IMD3) at the frequencies of $2 \omega_{2}-\omega_{1}$ and $2 \omega_{1}-\omega_{2}$.

The $\mathrm{SFDR}_{n}$ indicates the maximum output SNR without the need for filtering to eliminate the $n_{\mathrm{th}}$-order IMD spurious frequencies. The SFDR and other insights can be obtained by examining the output powers of fundamental, IMD2, and IMD3 signals at different input powers. The relation between the output and the input RF power in decibels for each frequency component is illustrated in Fig. 45, obtained from the two-tone test technique [239]. Straight lines are extrapolated from the measured data points (dotted). The extrapolated lines for IMD2 and IMD3 intercept with the extrapolated line for fundamental signal, generating the input (output) intercept points IIP $_{2}$ and $\mathrm{IIP}_{3}$, corresponding to output intercept points $\mathrm{OIP}_{2}$ and $\mathrm{OIP}_{3}$, respectively. One can find that the output power of the fundamental signal exhibits a linear slope of one to the input power, while the those of IMD2 and IMD3 show a slope of 2 and 3, due to the nature of second-order and third-order nonlinear responses, respectively. The $\mathrm{SFDR}_{2}$ and $\mathrm{SFDR}_{3}$ can be deduced from Fig. 45, given by

$$
\mathrm{SFDR}_{n}=\frac{n-1}{n}\left(\mathrm{IIP}_{n}-\mathrm{NF}+174\right)
$$

\section{Figure 44}

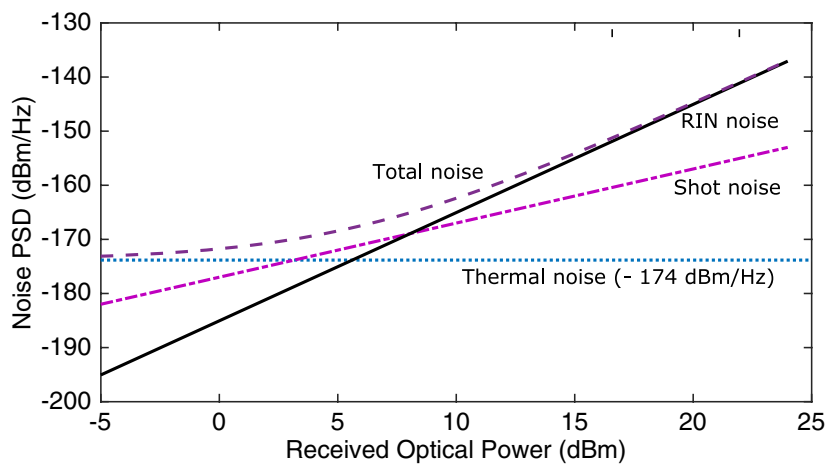

Calculated noise power density of the RIN noise, the shot noise, the thermal noise, and the total noise as a function of the received optical power in the photodetector. Parameters used in the simulations include the laser $\mathrm{RIN}=-160 \mathrm{dBc} / \mathrm{Hz}$ and the photodetector responsivity $r_{\mathrm{PD}}=0.5 \mathrm{~A} / \mathrm{W}$, with an impedance-matched photodetector. 
Detailed derivations of SFDR can be found in Refs. [1,6,10]. From Eq. (28), it is very convenient to use measurable figures of merits, such as the noise figure and the intercept points, to deduce SFDR.

\subsection{Challenges and Recent Progress in High-Performance IMWP Filters}

One of the main challenges that exists in IMWP filters is the poor RF link performance, compared to the link performance demonstrated in fiber-based MWP links. The performance gap is mainly introduced by the additional chip-to-fiber coupling loss. This increased RF link loss will immediately lead to deteriorations of the noise figure and SDFR, distancing IMWP filters from practical RF applications. Hence, effective approaches to improving the IMWP filter link performance must be explored.

Figure 46 compares the calculated link performance of a fiber-based MWP link and a chip-based IMWP link, respectively. Here, we assume that the integrated photonic chip introduces an additional optical insertion loss of $10 \mathrm{~dB}$, due the chip-to-fiber coupling. Figure 46(a) clearly shows a difference in the RF link gain of $20 \mathrm{~dB}$, which is translated from the additional $10 \mathrm{~dB}$ optical loss due to the square-law photodetection. The difference in the RF link gain leads to a large difference $(\sim 10 \mathrm{~dB})$ in the noise figure at a low optical power level where the dominant noise is the shot noise. Photonic chip packaging and improved chip coupling can certainly reduce the optical losses and thus improve the RF link gain. However, achieving a low noise figure and a high dynamic range is more challenging and critical, and cannot simply be solved by increasing RF link gain. For example, increasing optical source power and adding more optical amplifiers in a microwave photonic link will increase noise and thus will increase the noise figure, while inserting an RF amplifier before the modulator will reduce the dynamic range. As shown in Figs. 46(b) and 46(c), when the optical power is relatively high, the decreasing trend of the noise figure tends to be slow, as the RIN noise power becomes the dominant noise that limits the further reduction in noise figure (see Fig. 44). The SFDR 3 of the chip-based IMWP link reduces, mainly due to the degradation of the noise figure. Due to the losses and nonoptimized filter schemes, reported IMWP processors, typically IMWP filters, exhibit a link performance much

\section{Figure 45}

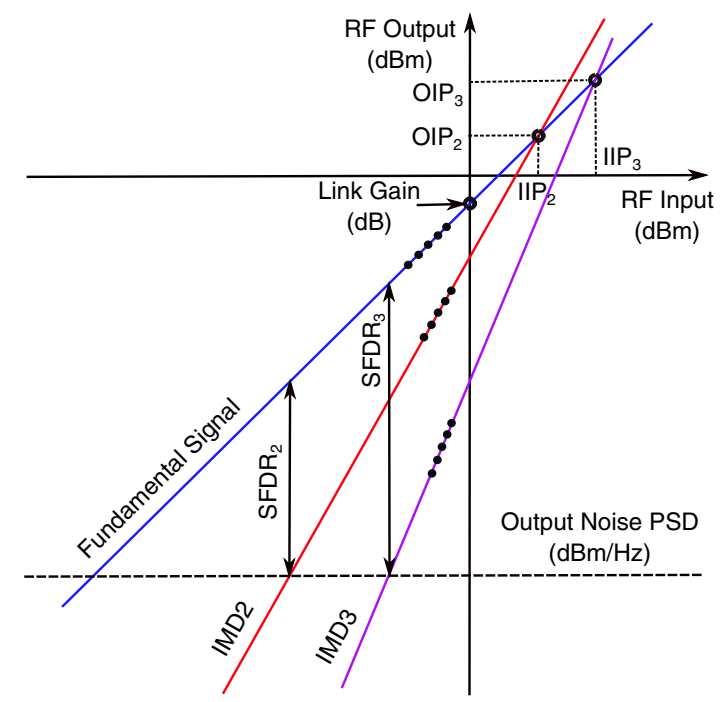

Schematic of SFDR definition. The output RF power (in decibels) of the fundamental signal and IMD components increases as a function of the input RF power (in decibels). The lines are extrapolated from the measured data indicated by the dots located on the lines. 
lower than the target performance, which has been optimized in fiber-based MWP links [1,240-243]. The demonstrated IMWP filters typically have an RF link gain lower than $-30 \mathrm{~dB}$ and a noise figure exceeding $30 \mathrm{~dB}[37,68,244]$. Therefore, innovative filter schemes, link performance optimization techniques, and nonlinear optical process need to be carefully considered to improve the overall link performance including link gain, noise figure, and dynamic range.

In order to compensate the optical insertion losses introduced by the integrated photonic chip, optical amplifiers and electrical amplifiers can be used to increase the RF link gain in the RF filter passbands. The first chip-based MWP filter with a high RF link gain [68] was achieved using a scheme shown in Fig. 47(a). In this work, a silicon photonic filter was utilized, introducing an intrinsic optical loss of $6 \mathrm{~dB}$ and a coupling loss of $9 \mathrm{~dB}$. These additional optical losses were offset by a subsequent optical amplifier before mixing with an optical oscillator with an optical power of $2.5 \mathrm{~mW}$. Moreover, the use of a balanced detection and two electrical amplifiers with $30 \mathrm{~dB}$ RF gain further increases the RF link gain to the level of $\approx 1 \mathrm{~dB}$, as shown in Fig. 47(b). However, the wideband noise generated by the optical amplifier placed after the photonic chip leads to a high noise figure of more than $40 \mathrm{~dB}$, resulting in a moderate $\mathrm{SFDR}_{3}$.

Apart from the coupling loss, the on-chip photonic signal processing usually introduces extra losses that further degrade the RF link gain. For instance, SSB modulation or selecting one sideband is commonly used in MWP filter implementations. Such a drawback was recently overcome by using a novel MWP filter scheme [245]. As introduced in Subsection 2.4b, using a cascaded transfer filter function, the novel filter scheme makes use of a synthesized $\pi$-phase-shift-only optical response on one optical sideband of an intensity-modulated signal, leading to highly selective RF cancellation in the stopband, while achieving enhanced filter passbands due to the constructive RF interference. Using this technique, MWP notch and bandstop filter responses with zero RF insertion loss were demonstrated, with $11 \mathrm{GHz}$ central frequency tuning

\section{Figure 46}

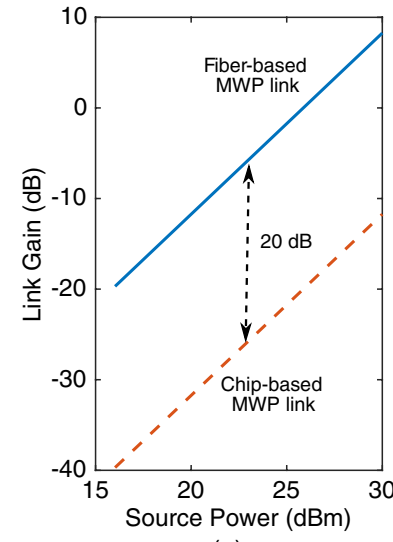

(a)

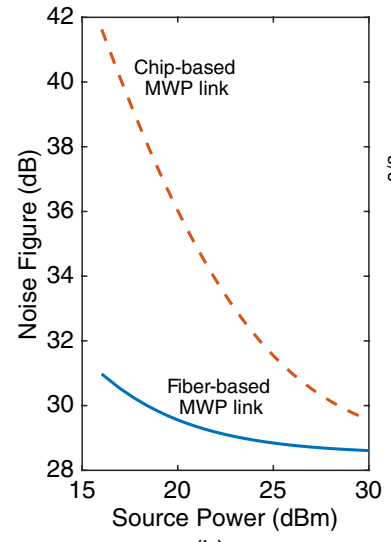

(b)

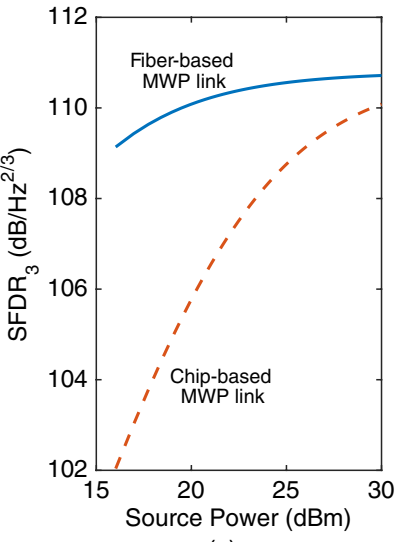

(c)

Simulated (a) RF link gain, (b) noise figure, and (c) $\mathrm{SFDR}_{3}$ for a fiber-based MWP link and a chip-based MWP link, respectively, as a function of optical source powers. In simulations, the chip-based MWP link exhibits an additional optical loss of $10 \mathrm{~dB}$, introduced by the chip-to-fiber coupling. Other parameters are maintained identical. Key parameters used for numerical simulations include the RF half-wave voltage $V_{\pi, R F}=3.8 \mathrm{~V}$, the laser RIN of $160 \mathrm{dBc} / \mathrm{Hz}$, the modulator insertion loss $L=3 \mathrm{~dB}$, quadrature biased MZM, and the photodetector responsivity $r_{P D}=0.5 \mathrm{~A} / \mathrm{W}$, with an impedance-matched photodetector. 
range. The notch filter has a $60 \mathrm{MHz}$ resolution and $>55 \mathrm{~dB}$ stopband suppression, while the bandstop filter has a nearly square stopband with $>30 \mathrm{~dB}$ suppression, as shown in Fig. 48. However, in this demonstration, the use of an optical amplifier placed right after the photonic chip resulted in a high noise figure of $>30 \mathrm{~dB}$.

In order to further minimize the performance gap, the noise figure of MWP systems is desirable to be reduced to a single-digit level [246,247]. A fully optimized chip-based

\section{Figure 47}
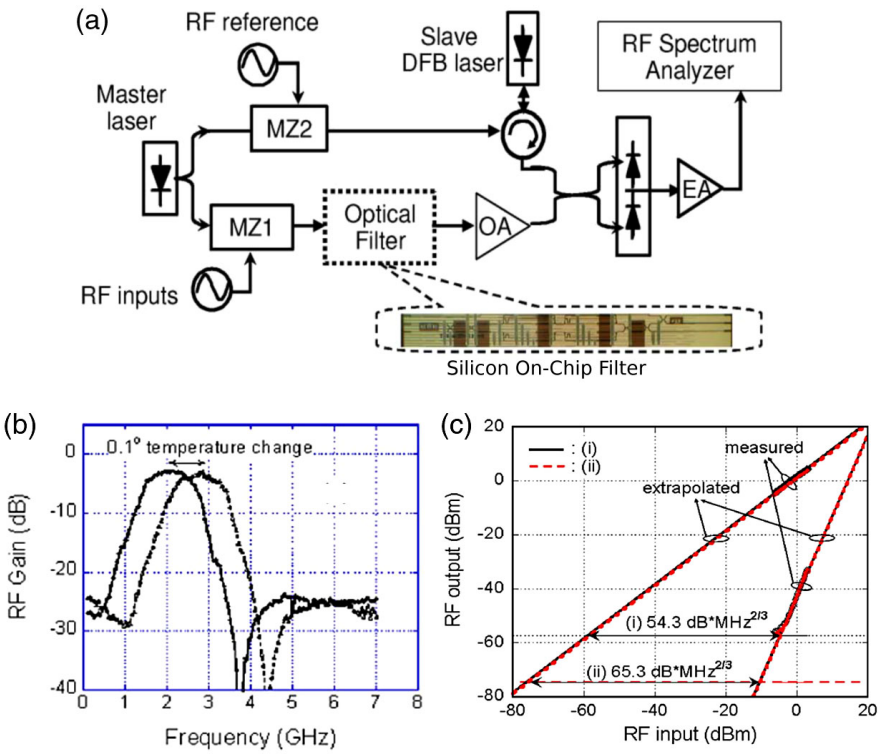

(a) Schematic of experimental setup with an inset showing the actual silicon on-chip photonic filter. (b) Measured RF filter response with a high RF link gain in the passband with central frequency tunability enabled by temperature tuning. (c) Measured overall RF performance. (C) 2010 IEEE. Reprinted, with permission, from Tu et al., J. Lightwave Technol. 28, 3019-3028 (2010) [68].

\section{Figure 48}

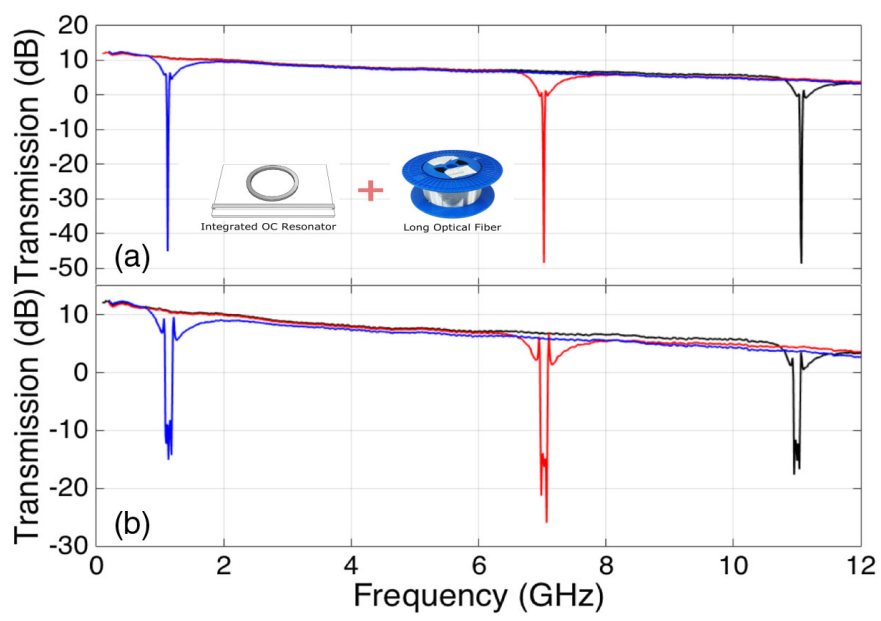

Measured spectra of (a) MWP notch filters and (b) MWP bandstop filters with different center frequencies. The 3-dB bandwidths of the notch and bandstop filters are 60 and $140 \mathrm{MHz}$, respectively. The inset shows $\mathrm{Si}_{3} \mathrm{~N}_{4}$ chip and 4.6-km-long optical fiber for construing a $\pi$-phase-shift-only optical response. Reprinted with permission from [64]. Copyright 2016 Optical Society of America. 
MWP notch filter was reported using the filter scheme introduced in Subsection 2.4b, based on dual-sideband processing and an intensity modulation scheme [41]. In order to minimize the noise power in the MWP filter link, the noise figure of the filter passbands was systematically investigated, when the locations of optical amplifiers were altered along the link [113], as shown in Fig. 49(a). This study suggests that an optical amplifier is preferably located between the low-biased intensity modulator and the integrated $\mathrm{Si}_{3} \mathrm{~N}_{4}$ photonic chip, yielding a minimum noise figure of $<20 \mathrm{~dB}$, as shown in Fig. 49(b). This level of the noise figure was achieved due to the faster increase of RF link gain than that of noise power [Eq. (24)], when the bias angle of the intensity modulator moves towards the null point [246]. Compared to other demonstrations of IMWP filters [30,37,64,68,78,111,248], a record-high RF link performance was achieved, featuring a maximum RF link gain of $8 \mathrm{~dB}$, a minimum noise figure of $15.6 \mathrm{~dB}$, and an overall $\mathrm{SFDR}_{3}$ of $116 \mathrm{~dB} \cdot \mathrm{Hz}^{2 / 3}$ [41,249], as shown in Fig. 49(c). Combined with the ultradeep stopband rejection and high spectral resolution, actual signal filtering experiment was performed, achieving $2 \mathrm{~dB}$ amplification for the signal of interest and $47 \mathrm{~dB}$ suppression on a strong unwanted interference with $250 \mathrm{MHz}$ separation, as shown in Figs. 49(d) and 49(e). This chip-based MWP notch filter scheme, for the first time, demonstrated the combination of high RF performance and advanced filter functionalities, which established the first step toward real-world applications of IMWP filters [250]. Using the same photonic platform, a positive-RF-gain RF photonic bandpass filter with tunable central frequencies

\section{Figure 49}

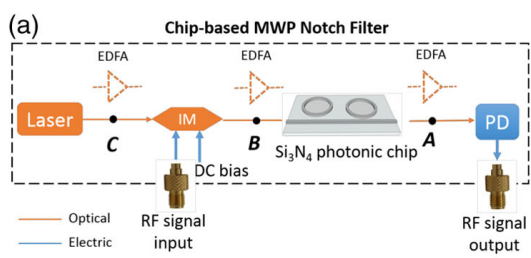

(c)
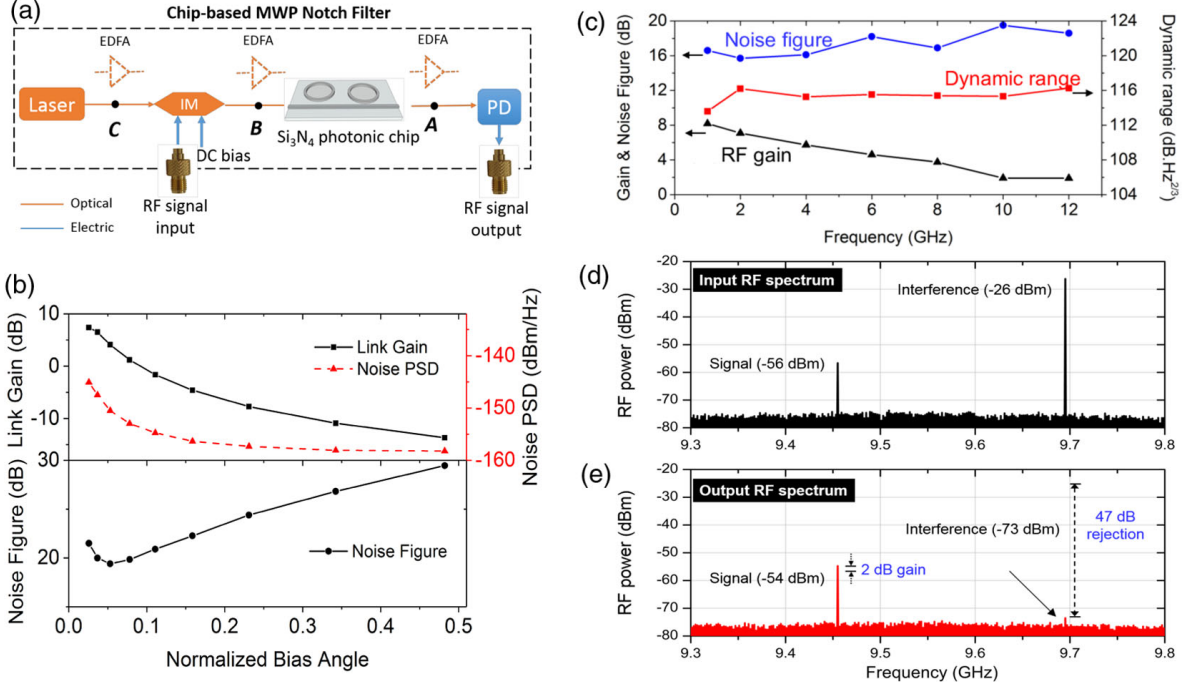

(a) Schematic of implementation of a $\mathrm{Si}_{3} \mathrm{~N}_{4}$-based MWP notch filter based on the RF-interference technique and DSB intensity modulation scheme. In the optical link, three locations (marked by A, B, and C) can be considered to accommodate the EDFA to improve the link RF performance. (b) Measured RF link gain, noise power spectrum density, and noise figure in the filter passband at $6 \mathrm{GHz}$, when EDFAs are located at Point B and Point C. (C) 2018 IEEE. Reprinted, with permission, from Liu et al., J. Lightwave Technol. 36, 4361-4370 (2018) [113]. (c) Measured overall RF link performance over the filter operation frequencies from 0 to $12 \mathrm{GHz}$. Measured electrical spectra of (d) input RF signals and (e) output RF signals, showing positive RF gain for the signal of interest and $47 \mathrm{~dB}$ suppression for the unwanted interference. IM, Intensity modulator; PD, photodetector. Black dots indicate the possible locations of optical amplifiers. EDFA, erbium-doped fiber amplifier. Reprinted with permission from [41] Copyright 2017 Optical Society of America. 
and multiple passbands was achieved, combined with a ring-enabled carrier control technique [63].

Recently, efforts have also been made to improve the RF link performance of onchip Brillouin-based MWP filters [60]. One approach taken utilizes the anti-Stokes components (loss resonances) of on-chip SBS to carve out an optical bandpass filter, as shown in Fig. 50(a). Therefore, the passband is free from the SBS-induced noise, resulting in a reduction of noise figure to $28 \mathrm{~dB}$ compared with other schemes, as shown in Fig. 50(b). Such a scheme was previously explored using fiber-based SBS for a low-noise optical filter [251] and a low-noise-figure MWP bandpass filter [252]. The suboptimal performance of the demonstration using on-chip SBS, on the other hand, is still limited by optical insertion losses from the SSB modulation scheme and the photonic circuits, only yielding an RF passband gain of $-15 \mathrm{~dB}$. However, the overall RF performance is possible to be improved using optimal modulation schemes [253].

Although the recent demonstrations of chip-based MWP filters have achieved appreciable RF link gain, optimized noise figure (NF) and SFDR have not completely met the target performance required for practical applications. One issue underlying the performance gap is the dominant intensity noise in the MWP link, which directly limits further reduction in noise figure and the increase of SFDR. The balanced detection technique is promising to minimize intensity noise, by subtracting the common intensity noises via a balanced detection configuration [240]. This approach is effective to shift the link from the RIN-noise-limited regime to the shot-noise-limited regime, resulting in a lower NF and a high SFDR.

For the emerging fully integrated microwave photonic filters, the link performance is crucial for future and existing RF systems, in conjunction with miniaturized footprints desired for size, weight, power and cost sensitive applications. Another equally important advantage of fully integrated photonic circuits is the negligible connection losses between on-chip components, which can improve the link gain and relax the requirement of high-power optical sources and amplifiers. Recent work investigated the feasibility of implementing a high-performance fully integrated MWP filter, numerically predicting the expected performance of on-chip components [113]. The calculations considered fully integrated MWP filter circuits

\section{Figure 50}
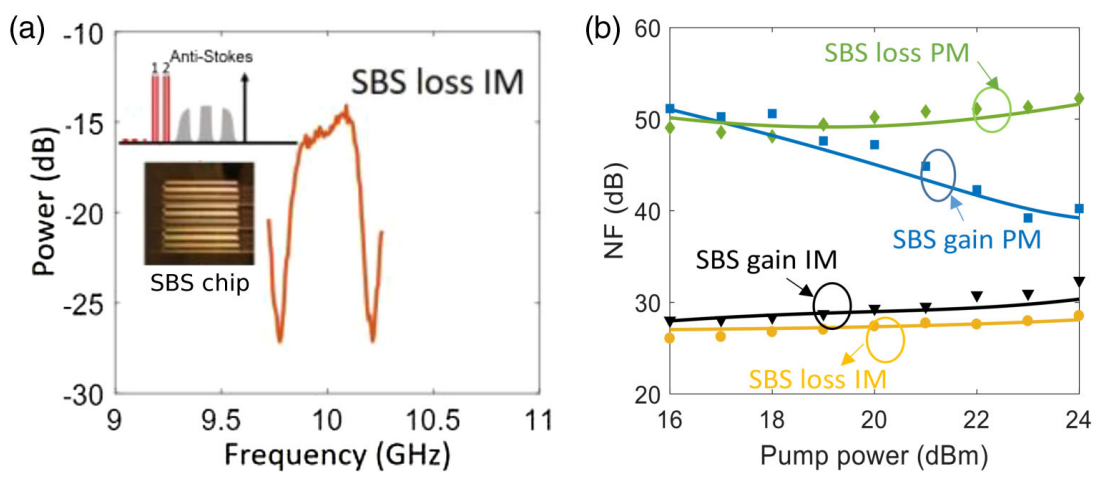

(a) Measured MWP bandpass filter response. The insets show the SBS-loss-based filter scheme and the $\mathrm{As}_{2} \mathrm{~S}_{3}$-based SBS photonic circuit, respectively. (b) Measured noise figure under various pump powers (at the chip facet) for different MWP filter schemes. On-chip pump power of $24 \mathrm{dBm}$ produces nearly $30 \mathrm{~dB}$ SBS gain. (C) 2019 IEEE. Reprinted, with permission, from Xie et al., J. Lightwave Technol. 37, 5246-5258 (2019) [60]. 


\section{Figure 51}

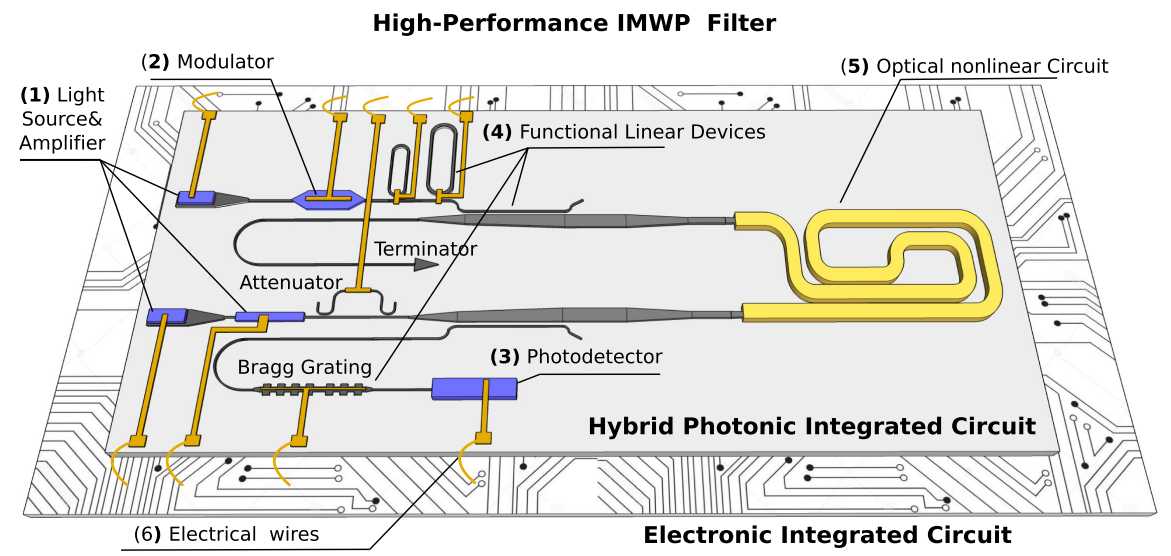

Conceptual diagram of a fully integrated high-performance IMWP filter implemented via heterogeneous/hybrid photonic integration technology.

that avoid the requirement for optical amplification stages, due to the negligible connection loss in the circuit. The calculations suggested an on-chip laser with an output of $150 \mathrm{~mW}$ and a RIN of $<-150 \mathrm{dBc} / \mathrm{Hz}$ to sustain a high RF link gain and a low RIN noise. An on-chip MZM with a low $V_{\pi, \mathrm{RF}}$ and an integrated photodetector with a high responsivity $\sim 1 \mathrm{~A} / \mathrm{W}$ are also desired to achieve a high $\mathrm{RF}$ link gain. It should be noted that the decrease of $V_{\pi, \mathrm{RF}}$ will lead to a lower dynamic range. Therefore, reducing $V_{\pi, \mathrm{RF}}$ should not be the only way to increase the RF link performance. The linearization of MZM will also play a key role to achieve a high SFDR for IMWP systems, which introduces a new freedom to optimize the overall link performance.

\section{CONCLUSION}

We have provided an overview of the basic concepts, configurations, and the evolution of integrated microwave photonic filters, and highlighted the attractive advantages of compactness and advanced functionalities that were previously unattainable in conventional fiber-based counterparts. We have reviewed the recent progress of IMWP from the perspectives of filtering functionalities, photonic integration, and key performance metrics, which are crucial factors to justify the radical usability of integrated microwave photonic filters in real-world applications. As the technical basis, widely adopted principles and link architectures for the implementation of integrated microwave photonic filters have been reviewed and summarized along with representative demonstrations based on integrated photonic devices and circuits, highlighting the advanced RF-interference technique. As a key photonic processing element, the integrated photonic devices and optical effects used in state-of-the-art integrated microwave photonic filters have been reviewed, highlighting the unprecedented advantages in terms of spectral resolution and compactness enabled by on-chip nonlinear optical processes such as SBS and Kerr microcombs. Finally, we reviewed the recent progress on achieving high-RF-performance integrated microwave photonic filters, which made important steps towards meeting the demanding performance requirements of practical RF and microwave applications. In the last decade, the rapid development in IMWP has addressed the challenges in spectral resolution, device compactness, and filter tunability, which were raised in the early review [6]. In this paper, we have taken a fresh look at the landscape of the field of integrated microwave photonic filters. 
Future research and development of integrated microwave photonic filters are expected to target the simultaneous synergy of optimal filtering schemes, novel photonic processing approaches using both linear and nonlinear optical devices, and advanced photonic integration technologies. Since there is no single material platform that can provide complete functions for fully integrated IMWP systems, solutions that involve the integration of multiple materials on a monolithic chip will be attractive. Figure 51 provides a conceptual vision of a monolithically integrated high-performance IMWP filter that can enable high RF link performance and advanced filtering functionality, controlled by an electronic integrated circuit. Such an envisaged IMWP filter circuit can be implemented through heterogeneous/hybrid photonic integration technologies, which combine active optoelectronic components such as light sources, modulators and photodetectors, programmable linear optical elements, optical mode multiplexers, and nonlinear photonic processing units. A high degree of photonic integration can significantly reduce the interconnection optical losses, which is important to achieve high RF performance, while sustaining versatile and programmable RF photonic signal processing capability.

To achieve this envisaged high-performance IMWP filter, challenges in on-chip device performance and signal processing schemes need to be addressed to compete with the commercial off-chip devices [40]. However, these challenges can be overcome by the recent developments and breakthroughs in high-performance on-chip photonic devices. These recent breakthroughs include chip-based lownoise [228,254,255] and high-power $(>100 \mathrm{~mW})$ [150,225,256] light sources; on-chip E-O modulators with ultralow- $\mathrm{V}_{\pi, \mathrm{RF}}(<1.4$ volts) [257,258], high-linearity InP/Si (an SFDR up to $117.5 \mathrm{~dB} . \mathrm{Hz}^{2 / 3}$ ) [259], and ultrawide analog bandwidth $(>100 \mathrm{GHz})$ [260]; negligible interconnection loss $(\sim 0.2 \mathrm{~dB})$ between difference materials through vertical directional couplers and adiabatic tapers [224,261,262]; and ultralow-loss on-chip photonic links $(<0.1 \mathrm{~dB} / \mathrm{cm})$ such as wide silicon waveguides [124-126], $\mathrm{Si}_{3} \mathrm{~N}_{4}$ waveguides [114,128,263], and $\mathrm{LiNbO}_{3}$ rib waveguides [200]. Among available material platforms, the emerging ultralow-loss $\mathrm{LiNbO}_{3}$ photonic circuit is able to offer ultralow-loss waveguides, a high-performance integrated $\mathrm{LiNbO}_{3}$ modulator, $\chi_{2}$-enabled ultrafast tunable photonic devices, and strong optical nonlinear effects [197-199]. With such superior properties, the integrated $\mathrm{LiNbO}_{3}$ photonic platform is promising to offer new possibilities of IMWP filters in the future. These advanced PIC technologies and novel material platforms will enable the future proliferation of high-performance IMWP filters.

\section{FUNDING}

Australian Research Council (CE110001018, DE150101535, DE170100585, FL120100029); Air Force Office of Scientific Research (FA2386-16-1-4036); Lockheed Martin; Office of Naval Research Global (N62909-18-1-2013); Science and Engineering Research Board (SRG/2019/00 1632).

\section{REFERENCES}

1. V. J. Urick, J. D. Mckinney, and K. J. Williams, Fundamentals of Microwave Photonics (Wiley, 2015).

2. R. J. Cameron, C. M. Kudsia, and R. R. Mansour, Microwave Filters for Communication Systems (Wiley, 2018), Vol. 43.

3. J. Capmany and D. Novak, "Microwave photonics combines two worlds," Nat. Photonics 1, 319-330 (2007).

4. A. J. Seeds and K. J. Williams, "Microwave photonics," J. Lightwave Technol. 24, 4628-4641 (2006). 
5. J. Yao, “Microwave photonics,” J. Lightwave Technol. 27, 314-335 (2009).

6. J. Capmany, B. Ortega, and D. Pastor, "A tutorial on microwave photonic filters," J. Lightwave Technol. 24, 201-229 (2006).

7. J. Witzens, "Silicon photonics: modulators make efficiency leap," Nat. Photonics 11, 459-462 (2017).

8. R. Baets, A. Z. Subramanian, S. Clemmen, B. Kuyken, P. Bienstman, N. Le Thomas, G. Roelkens, D. Van Thourhout, P. Helin, and S. Severi, "Silicon photonics: silicon nitride versus silicon-on-insulator," in Optical Fiber Communication Conference (OSA, 2016), paper Th3J.1.

9. R. Nagarajan, M. Kato, J. Pleumeekers, P. Evans, S. Corzine, S. Hurtt, A. Dentai, S. Murthy, M. Missey, R. Muthiah, R. A. Salvatore, C. Joyner, R. Schneider, M. Ziari, F. Kish, and D. Welch, "InP photonic integrated circuits," IEEE J. Sel. Top. Quantum Electron. 16, 1113-1125 (2010).

10. D. Marpaung, C. Roeloffzen, R. Heideman, A. Leinse, S. Sales, and J. Capmany, "Integrated microwave photonics," Laser Photon. Rev. 7, 506-538 (2013).

11. W. Zhang and J. Yao, "Silicon-based integrated microwave photonics," IEEE J. Quantum Electron. 52, 0600412 (2016).

12. D. Marpaung, J. Yao, and J. Capmany, "Integrated microwave photonics," Nat. Photonics 13, 80-90 (2019).

13. D. Jachowski, "Compact, frequency-agile, absorptive bandstop filters," in IEEE MTT-S International Microwave Symposium Digest (IEEE, 2005), pp. 513-516.

14. S. Sun, J. Shi, L. Zhu, S. Rustagi, and K. Kang, and K. Mouthaan, "40 GHz compact TFMS meander-line bandpass filter on silicon substrate," Electron. Lett. 43, 1433-1434 (2007).

15. Z. Gao, J. Ma, M. Yu, and Y. Ye, "A fully integrated CMOS active bandpass filter for multiband RF front-ends," IEEE Trans. Circuits Syst. II, Exp. Briefs 55, 718-722 (2008).

16. J. L. Lopez, J. Verd, A. Uranga, J. Giner, G. Murillo, F. Torres, G. Abadal, and N. Barniol, "A CMOS-MEMS RF-tunable bandpass filter based on two high-Q 22-MHz polysilicon clamped-clamped beam resonators," IEEE Electron Device Lett. 30, 718-720 (2009).

17. P. W. Wong and I. Hunter, "Electronically reconfigurable microwave bandpass filter," IEEE Trans. Microwave Theory Tech. 57, 3070-3079 (2009).

18. K. Y. Lee and G. M. Rebeiz, "A miniature 8-16 GHz packaged tunable frequency and bandwidth RF MEMS filter," in IEEE International Symposium on Radio-Frequency Integration Technology (RFIT) (2009), pp. 249-252.

19. S. Courrèges, Y. Li, Z. Zhao, K. Choi, A. Hunt, S. Horst, J. D. Cressler, and J. Papapolymerou, "A Ka-band electronically tunable ferroelectric filter," IEEE Microwave Wireless Compon. Lett. 19, 356-358 (2009).

20. I. Reines, S. J. Park, and G. M. Rebeiz, "Compact low-loss tunable $X$-band bandstop filter with miniature RF-MEMS switches," IEEE Trans. Microwave Theory Tech. 58, 1887-1895 (2010).

21. K. Y. Yuk, S. Fouladi, R. Ramer, and R. R. Mansour, "RF MEMS switchable interdigital bandpass filter," IEEE Microwave Wireless Compon. Lett. 22, 44-46 (2012).

22. T. Snow, J. Lee, and W. J. Chappell, "Tunable high quality-factor absorptive bandstop filter design," in IEEE/MTT-S International Microwave Symposium Digest (IEEE, 2012), pp. 1-3.

23. B. Yassini, M. Yu, and B. Keats, "A Ka-band fully tunable cavity filter," IEEE Trans. Microwave Theory Tech. 60, 4002-4012 (2012).

24. B. Kim, J. Lee, J. Lee, B. Jung, and W. J. Chappell, "RF CMOS integrated onchip tunable absorptive bandstop filter using Q-tunable resonators," IEEE Trans. Electron Devices 60, 1730-1737 (2013). 
25. E. J. Naglich, A. C. Guyette, and D. Peroulis, "High-Q intrinsically-switched quasi-absorptive tunable bandstop filter with electrically-short resonators," in IEEE MTT-S International Microwave Symposium Digest (2014), pp. 2-5.

26. Z. Yang and D. Peroulis, "A 23-35 GHz MEMS tunable all-silicon cavity filter with stability characterization up to 140 million cycles," in IEEE MTT-S International Microwave Symposium Digest (2014), pp. 1-4.

27. C. Arnold, J. Parlebas, and T. Zwick, "Reconfigurable waveguide filter with variable bandwidth and center frequency," IEEE Trans. Microwave Theory Tech. 62, 1663-1670 (2014).

28. C. Arnold, J. Parlebas, and T. Zwick, "Center frequency and bandwidth tunable waveguide bandpass filter with transmission zeros," in European Microwave Week 2015: "Freedom Through Microwaves," (EuMW) 2015-Conference Proceedings; 2015 45th European Microwave Conference Proceedings (EuMC) (2015), pp. 1220-1223.

29. S. Yegnanarayanan, P. D. Trinh, F. Coppinger, and B. Jalali, "Compact siliconbased integrated optical time-delay network," Proc. SPIE 3160, 2-10 (1997).

30. M. Rasras, K.-Y. Tu, D. Gill, Y.-K. Chen, A. White, S. Patel, A. Pomerene, D. Carothers, J. Beattie, M. Beals, J. Michel, and L. Kimerling, "Demonstration of a tunable microwave-photonic notch filter using low-loss silicon ring resonators," J. Lightwave Technol. 27, 2105-2110 (2009).

31. X. Wang, W. Shi, H. Yun, S. Grist, N. A. F. Jaeger, and L. Chrostowski, "Narrow-band waveguide Bragg gratings on SOI wafers with CMOS-compatible fabrication process," Opt. Express 20, 15547-15558 (2012).

32. E. J. Norberg, R. S. Guzzon, J. S. Parker, L. A. Johansson, and L. A. Coldren, "Programmable photonic microwave filters monolithically integrated in InP-InGaAsP,” J. Lightwave Technol. 29, 1611-1619 (2011).

33. J. Sancho, J. Bourderionnet, J. Lloret, S. Combrié, I. Gasulla, S. Xavier, S. Sales, P. Colman, G. Lehoucq, D. Dolfi, J. Capmany, and A. De Rossi, "Integrable microwave filter based on a photonic crystal delay line," Nat. Commun. 3, 1075 (2012).

34. A. Byrnes, R. Pant, E. Li, D.-Y. Choi, C. G. Poulton, S. Fan, S. Madden, B. Luther-Davies, and B. J. Eggleton, "Photonic chip based tunable and reconfigurable narrowband microwave photonic filter using stimulated Brillouin scattering," Opt. Express 20, 18836-18845 (2012).

35. X. Xue, Y. Xuan, H. J. Kim, J. Wang, D. E. Leaird, M. Qi, and A. M. Weiner, "Programmable single-bandpass photonic RF filter based on Kerr comb from a microring," J. Lightwave Technol. 32, 3557-3565 (2014).

36. T. G. Nguyen, M. Shoeiby, S. T. Chu, B. E. Little, R. Morandotti, A. Mitchell, and D. J. Moss, "Integrated frequency comb source based Hilbert transformer for wideband microwave photonic phase analysis," Opt. Express 23, 22087-22097 (2015).

37. D. Marpaung, B. Morrison, M. Pagani, R. Pant, D.-Y. Choi, B. Luther-Davies, S. J. Madden, and B. J. Eggleton, "Low-power, chip-based stimulated Brillouin scattering microwave photonic filter with ultrahigh selectivity," Optica 2, 76-83 (2015).

38. L. Zhuang, C. G. H. Roeloffzen, M. Hoekman, K.-J. Boller, and A. J. Lowery, "Programmable photonic signal processor chip for radiofrequency applications," Optica 2, 854-859 (2015).

39. W. Liu, M. Li, R. S. Guzzon, E. J. Norberg, J. S. Parker, M. Lu, L. A. Coldren, and J. Yao, "A fully reconfigurable photonic integrated signal processor," Nat. Photonics 10, 190-195 (2016).

40. J. S. Fandiño, P. Muñoz, D. Doménech, and J. Capmany, "A monolithic integrated photonic microwave filter," Nat. Photonics 11, 124-129 (2017). 
41. Y. Liu, J. Hotten, A. Choudhary, B. J. Eggleton, and D. Marpaung, "Alloptimized integrated RF photonic notch filter," Opt. Lett. 42, 4631-4634 (2017).

42. D. Pérez, I. Gasulla, L. Crudgington, D. J. Thomson, A. Z. Khokhar, K. Li, W. Cao, G. Z. Mashanovich, and J. Capmany, "Multipurpose silicon photonics signal processor core," Nat. Commun. 8, 636 (2017).

43. Y. Liu, A. Choudhary, G. Ren, K. Vu, B. Morrison, A. Casas-Bedoya, T. G. Nguyen, D.-Y. Choi, P. Ma, A. Mitchell, S. J. Madden, D. Marpaung, and B. J. Eggleton, "Integration of Brillouin and passive circuits for enhanced radio-frequency photonic filtering," APL Photon. 4, 106103 (2019).

44. X. Xu, M. Tan, J. Wu, T. G. Nguyen, S. T. Chu, B. E. Little, R. Morandotti, A. Mitchell, and D. J. Moss, "Advanced adaptive photonic RF filters with 80 taps based on an integrated optical micro-comb source," J. Lightwave Technol. 37, 1288-1295 (2019).

45. R. Soref and J. Lorenzo, "Single-crystal silicon: a new material for 1.3 and $1.6 \mu \mathrm{m}$ integrated-optical components," Electron. Lett. 21, 953-954 (1985).

46. R. A. Soref and B. R. Bennett, "Electrooptical effects in silicon," IEEE J. Quantum Electron. 23, 123-129 (1987).

47. B. R. Bennett, R. A. Soref, and J. A. Del Alamo, "Carrier-induced change in refractive index of InP, GaAs, and InGaAsP," IEEE J. Quantum Electron. 26, 113-122 (1990).

48. E. J. Norberg, R. S. Guzzon, S. C. Nicholes, J. S. Parker, and L. A. Coldren, "Programmable photonic lattice filters in InGaAsP-InP," IEEE Photonics Technol. Lett. 22, 109-111 (2010).

49. L. Zhuang, D. Marpaung, M. Burla, W. Beeker, A. Leinse, and C. Roeloffzen, "Low-loss, high-index-contrast $\mathrm{Si}_{3} \mathrm{~N}_{4} / \mathrm{SiO}_{2}$ optical waveguides for optical delay lines in microwave photonics signal processing," Opt. Express 19, 23162-23170 (2011).

50. M. Burla, D. Marpaung, L. Zhuang, C. Roeloffzen, M. R. Khan, A. Leinse, M. Hoekman, and R. Heideman, "On-chip CMOS compatible reconfigurable optical delay line with separate carrier tuning for microwave photonic signal processing," Opt. Express 19, 21475-21484 (2011).

51. C. Roeloffzen, L. Zhuang, C. Taddei, A. Leinse, R. G. Heideman, P. W. L. van Dijk, R. M. Oldenbeuving, D. A. I. Marpaung, M. Burla, and K. J. Boller, "Silicon nitride microwave photonic circuits," Opt. Express 21, 22937-22961 (2013).

52. J. Wu, X. Xu, T. G. Nguyen, S. T. Chu, B. E. Little, R. Morandotti, A. Mitchell, and D. J. Moss, "RF photonics: an optical microcombs' perspective," IEEE J. Sel. Top. Quantum Electron. 24, 1-20 (2018).

53. X. Xu, M. Tan, J. Wu, T. G. Nguyen, S. T. Chu, B. E. Little, R. Morandotti, A. Mitchell, and D. J. Moss, "High performance RF filters via bandwidth scaling with Kerr micro-combs," APL Photon. 4, 026102 (2019).

54. Y. Liu, A. Choudhary, G. Ren, K. Vu, B. Morrison, A. Casas-Bedoya, T. G. Nguyen, D.-Y. Choi, A. Mitchell, S. J. Madden, D. Marpaung, and B. J. Eggleton, "Integrating Brillouin processing with functional circuits for enhanced $\mathrm{RF}$ photonic processing," in International Topical Meeting on Microwave Photonics (MWP) (IEEE, 2018), pp. 1-4.

55. Q. Sun, L. Zhou, L. Lu, G. Zhou, and J. Chen, "Reconfigurable high-resolution microwave photonic filter based on dual-ring-assisted MZIs on the $\mathrm{Si}_{3} \mathrm{~N}_{4}$ platform," IEEE Photon. J. 10, 1-12 (2018).

56. R. S. Guzzon, E. J. Norberg, J. S. Parker, L. A. Johansson, and L. A. Coldren, "Integrated InP-InGaAsP tunable coupled ring optical bandpass filters with zero insertion loss," Opt. Express 19, 7816-7826 (2011). 
57. B. Guan, S. S. Djordjevic, N. K. Fontaine, L. Zhou, S. Ibrahim, R. P. Scott, D. J. Geisler, Z. Ding, and S. J. Ben Yoo, "CMOS compatible reconfigurable silicon photonic lattice filters using cascaded unit cells for RF-photonic processing," IEEE J. Sel. Top. Quantum Electron. 20, 359-368 (2014).

58. J. Dong, L. Liu, D. Gao, Y. Yu, A. Zheng, T. Yang, and X. Zhang, "Compact notch microwave photonic filters using on-chip integrated microring resonators," IEEE Photon. J. 5, 5500307 (2013).

59. J. Palací, G. E. Villanueva, J. V. Galán, J. Martí, and B. Vidal, "Single bandpass photonic microwave filter based on a notch ring resonator," IEEE Photon. Technol. Lett. 22, 1276-1278 (2010).

60. Y. Xie, A. Choudhary, Y. Liu, D. Marpaung, K. Vu, P. Ma, D.-Y. Choi, S. Madden, and B. J. Eggleton, "System-level performance of chip-based Brillouin microwave photonic bandpass filters," J. Lightwave Technol. 37, 5246-5258 (2019).

61. X. Xu, J. Wu, T. G. Nguyen, M. Shoeiby, S. T. Chu, B. E. Little, R. Morandotti, A. Mitchell, and D. J. Moss, "Advanced RF and microwave functions based on an integrated optical frequency comb source," Opt. Express 26, 2569-2583 (2018).

62. P. Zheng, H. Hong, J. Li, G. Hu, B. Yun, and Y. Cui, "Performances of microwave photonic notch filter based on microring resonator with dual-drive modulator," IEEE Photon. J. 11, 5500313 (2019).

63. Z. Zhu, Y. Liu, M. Merklein, O. Daulay, D. Marpaung, and B. J. Eggleton, "Positive link gain microwave photonic bandpass filter using $\mathrm{Si}_{3} \mathrm{~N}_{4}$-ring-enabled sideband filtering and carrier suppression," Opt. Express 27, 31727-31740 (2019).

64. Y. Liu, D. Marpaung, A. Choudhary, and B. J. Eggleton, "Lossless and high-resolution RF photonic notch filter," Opt. Lett. 41, 5306-5309 (2016).

65. S. Song, S. X. Chew, X. Yi, L. Nguyen, and R. A. Minasian, "Tunable singlepassband microwave photonic filter based on integrated optical double notch filter," J. Lightwave Technol. 36, 4557-4564 (2018).

66. A. Choudhary, I. Aryanfar, S. Shahnia, B. Morrison, K. Vu, S. Madden, B. Luther-Davies, D. Marpaung, and B. J. Eggleton, "Tailoring of the Brillouin gain for on-chip widely tunable and reconfigurable broadband microwave photonic filters," Opt. Lett. 41, 436-439 (2016).

67. H. Qiu, X. Zhang, F. Zhou, J. Qie, Y. Yao, X. Hu, Y. Zhang, X. Xiao, Y. Yu, and J. Dong, "A continuously tunable sub-gigahertz microwave photonic bandpass filter based on an ultra-high-Q silicon microring resonator," J. Lightwave Technol. 36, 4312-4318 (2018).

68. K. Y. Tu, M. S. Rasras, D. M. Gill, S. S. Patel, Y. K. Chen, A. E. White, A. Pomerene, D. Carothers, J. Beattie, M. Beals, J. Michel, and L. C. Kimerling, "Silicon RF-photonic filter and down-converter," J. Lightwave Technol. 28, 3019-3028 (2010).

69. P. Alipour, A. A. Eftekhar, A. H. Atabaki, Q. Li, S. Yegnanarayanan, C. K. Madsen, and A. Adibi, "Fully reconfigurable compact RF photonic filters using high- $Q$ silicon microdisk resonators," Opt. Express 19, 15899-15907 (2011).

70. L. Zhuang, "Flexible RF filter using a nonuniform SCISSOR," Opt. Lett. 41, 1118-1121 (2016).

71. S. X. Chew, X. Yi, S. Song, L. Li, P. Bian, L. Nguyen, and R. A. Minasian, "Silicon-on-insulator dual-ring notch filter for optical sideband suppression and spectral characterization," J. Lightwave Technol. 34, 4705-4713 (2016).

72. E. A. Kittlaus, P. Kharel, N. T. Otterstrom, Z. Wang, and P. T. Rakich, "RFphotonic filters via on-chip photonic-phononic emit-receive operations," J. Lightwave Technol. 36, 2803-2809 (2018). 
73. A. Casas-Bedoya, B. Morrison, M. Pagani, D. Marpaung, and B. J. Eggleton, "Tunable narrowband microwave photonic filter created by stimulated Brillouin scattering from a silicon nanowire," Opt. Lett. 40, 4154-4157 (2015).

74. L. Li, S. X. Chew, S. Song, K. Powell, X. Yi, L. Nguyen, and R. Minasian, "Reflective microring sensing probe based on narrowband microwave photonic notch filter," in International Topical Meeting on Microwave Photonics (MWP) (IEEE, 2018), pp. 1-4.

75. Y. Long and J. Wang, "Ultra-high peak rejection notch microwave photonic filter using a single silicon microring resonator," Opt. Express 23, 17739-17750 (2015).

76. D. Marpaung, B. Morrison, R. Pant, C. Roeloffzen, A. Leinse, M. Hoekman, R. Heideman, and B. J. Eggleton, " $\mathrm{Si}_{3} \mathrm{~N}_{4}$ ring resonator-based microwave photonic notch filter with an ultrahigh peak rejection," Opt. Express 21, 23286-23294 (2013).

77. B. Morrison, D. Marpaung, R. Pant, E. Li, D.-Y. Choi, S. Madden, B. LutherDavies, and B. J. Eggleton, "Tunable microwave photonic notch filter using on-chip stimulated Brillouin scattering," Opt. Commun. 313, 85-89 (2014).

78. X. Liu, Y. Yu, H. Tang, L. Xu, J. Dong, and X. Zhang, "Silicon-on-insulatorbased microwave photonic filter with narrowband and ultrahigh peak rejection," Opt. Lett. 43, 1359-1362 (2018).

79. X. Xu, J. Wu, M. Tan, T. G. Nguyen, S. T. Chu, B. E. Little, R. Morandotti, A. Mitchell, and D. J. Moss, "Orthogonally polarized RF optical single sideband generation and dual-channel equalization based on an integrated microring resonator," J. Lightwave Technol. 36, 4808-4818 (2018).

80. C. Taddei, L. Zhuang, M. Hoekman, A. Leinse, R. Oldenbeuving, P. van Dijk, and C. Roeloffzen, "Fully reconfigurable coupled ring resonator-based bandpass filter for microwave signal processing," in International Topical Meeting on Microwave Photonics (MWP) and the 2014 9th Asia-Pacific Microwave Photonics Conference (APMP) (IEEE, 2014), pp. 44-47.

81. K. J. Williams, "Signal processing subsystems for RF photonics," in Optical Fiber Communication Conference (OSA, 2017), paper W4B.1.

82. C. Lim, A. Nirmalathas, M. Bakaul, P. Gamage, K. L. Lee, Y. Yang, D. Novak, and R. Waterhouse, "Fiber-wireless networks and subsystem technologies," J. Lightwave Technol. 28, 390-405 (2010).

83. R. W. Boyd, Nonlinear Optics (Elsevier, 2003).

84. U. Cummings, "Linearized and high frequency electrooptic modulators," Ph.D. thesis (Caltech, 2005).

85. P. Dong, J. H. Sinsky, and C. Gui, "Coplanar-waveguide-based silicon MachZehnder modulator using a meandering optical waveguide and alternating-side PN junction loading," Opt. Lett. 41, 4401-4404 (2016).

86. D. Marpaung, B. Morrison, M. Pagani, R. Pant, and B. J. Eggleton, "Ultra-high suppression microwave photonic bandstop filters," Chin. Sci. Bull. 59(22), 2684-2692 (2014).

87. R. Tucker and D. Pope, "Microwave circuit models of semiconductor injection lasers," IEEE Trans. Microwave Theory Tech. 31, 289-294 (1983).

88. A. V. Oppenheim, Discrete-Time Signal Processing (Pearson, 1999).

89. J. Capmany, B. Ortega, D. Pastor, and S. Sales, "Discrete-time optical processing of microwave signals," J. Lightwave Technol. 23, 702-723 (2005).

90. C. K. Madsen and J. H. Zhao, Optical Filter Design and Analysis, Vol. 3 of Wiley Series in Microwave and Optical Engineering (Wiley, 1999).

91. J. Yao, "Photonics to the rescue: a fresh look at microwave photonic filters," IEEE Microwave Mag. 16(8), 46-60 (2015). 
92. J. Wang, R. Ashrafi, R. Adams, I. Glesk, I. Gasulla, J. Capmany, and L. R. Chen, "Subwavelength grating enabled on-chip ultra-compact optical true time delay line," Sci. Rep. 6, 30235 (2016).

93. J. Capmany, J. Cascón, J. L. Martín, S. Sales, D. Pastor, and J. Martí, “Synthesis of fiber-optic delay line filters," J. Lightwave Technol. 13, 2003-2012 (1995).

94. M. Sagues, R. García Olcina, A. Loayssa, S. Sales, and J. Capmany, "Multi-tap complex-coefficient incoherent microwave photonic filters based on optical single-sideband modulation and narrow band optical filtering," Opt. Express 16, 295-303 (2008).

95. W. Zhang, G. Yu, and J. Williams, "Tap multiplexed fibre grating-based optical transversal filter," Electron. Lett. 36, 1708-1710 (2000).

96. J. Mora, B. Ortega, J. Capmany, J. L. Cruz, M. V. Andres, D. Pastor, and S. Sales, "Automatic tunable and reconfigurable fiberoptic microwave filters based on a broadband optical source sliced by uniform fiber Bragg gratings," Opt. Express 10, 1291-1298 (2002).

97. J. Mora, B. Ortega, A. Diez, J. L. Cruz, M. V. Andrés, J. Capmany, and D. Pastor, "A single bandpass tunable photonic transversal filter based on a broadband optical source and a Mach-Zehnder interferometer," in MWP 2003 Proceedings International Topical Meeting on Microwave Photonics (2003), pp. 251-254.

98. B. A. L. Gwandu, W. Zhang, J. A. R. Williams, L. Zhang, and I. Bennion, "Microwave photonic filtering using Gaussian-profiled superstructured fibre Bragg grating and dispersive fibre," Electron. Lett. 38, 1328-1330 (2002).

99. E. Hamidi, D. E. Leaird, and A. M. Weiner, "Tunable programmable microwave photonic filters based on an optical frequency comb," IEEE Trans. Microwave Theory Tech. 58, 3269-3278 (2010).

100. V. R. Supradeepa, C. M. Long, R. Wu, F. Ferdous, E. Hamidi, D. E. Leaird, and A. M. Weiner, "Comb-based radiofrequency photonic filters with rapid tunability and high selectivity," Nat. Photonics 6, 186-194 (2012).

101. A. L. Gaeta, M. Lipson, and T. J. Kippenberg, "Photonic-chip-based frequency combs," Nat. Photonics 13, 158-169 (2019).

102. S. Liao, Y. Ding, J. Dong, T. Yang, X. Chen, D. Gao, and X. Zhang, “Arbitrary waveform generator and differentiator employing an integrated optical pulse shaper," Opt. Express 23, 12161-12173 (2015).

103. X. Xu, J. Wu, M. Shoeiby, T. G. Nguyen, S. T. Chu, B. E. Little, R. Morandotti, A. Mitchell, and D. J. Moss, "Reconfigurable broadband microwave photonic intensity differentiator based on an integrated optical frequency comb source," APL Photon. 2, 096104 (2017).

104. T. G. Nguyen, M. Shoeiby, S. T. Chu, R. Morandotti, A. Mitchell, and D. J. Moss, "Quadrature hybrid RF photonic coupler using an integrated frequency comb source," in Integrated Photonics Research, Silicon and Nanophotonics (IPRSN) (2015), paper IT2B.4.

105. M. Tan, X. Xu, B. Corcoran, J. Wu, A. Boes, T. G. Nguyen, S. T. Chu, B. E. Little, R. Morandotti, A. Mitchell, and D. J. Moss, "Microwave and RF photonic fractional Hilbert transformer based on a $50 \mathrm{GHz}$ Kerr micro-comb," J. Lightwave Technol. 37, 6097-6104 (2019).

106. A. J. Metcalf, H.-J. Kim, D. E. Leaird, J. A. Jaramillo-Villegas, K. A. McKinzie, V. Lal, A. Hosseini, G. E. Hoefler, F. Kish, and A. M. Weiner, "Integrated line-by-line optical pulse shaper for high-fidelity and rapidly reconfigurable RF-filtering," Opt. Express 24, 23925-23940 (2016).

107. L. Liu, F. Jiang, S. Yan, S. Min, M. He, D. Gao, and J. Dong, "Photonic measurement of microwave frequency using a silicon microdisk resonator," Opt. Commun. 335, 266-270 (2015). 
108. J. Wu, J. Peng, B. Liu, T. Pan, H. Zhou, J. Mao, Y. Yang, C. Qiu, and Y. Su, "Passive silicon photonic devices for microwave photonic signal processing," Opt. Commun. 373, 44-52 (2015).

109. W. Zhang and R. A. Minasian, "Widely tunable single-passband microwave photonic filter based on stimulated Brillouin scattering," IEEE Photon. Technol. Lett. 23, 1775-1777 (2011).

110. W. Zhang and R. A. Minasian, "Switchable and tunable microwave photonic Brillouin-based filter," IEEE Photon. J. 4, 1443-1455 (2012).

111. D. Marpaung, B. Morrison, R. Pant, and B. J. Eggleton, "Frequency agile microwave photonic notch filter with anomalously high stopband rejection," Opt. Lett. 38, 4300-4303 (2013).

112. Y. Liu, A. Choudhary, D. Marpaung, and B. J. Eggleton, "Gigahertz optical tuning of an on-chip radio frequency photonic delay line," Optica 4, 418-423 (2017).

113. Y. Liu, D. Marpaung, A. Choudhary, J. Hotten, and B. J. Eggleton, "Link performance optimization of chip-based $\mathrm{Si}_{3} \mathrm{~N}_{4}$ microwave photonic filters," J. Lightwave Technol. 36, 4361-4370 (2018).

114. C. G. H. Roeloffzen, M. Hoekman, E. J. Klein, L. S. Wevers, R. B. Timens, D. Marchenko, D. Geskus, R. Dekker, A. Alippi, R. Grootjans, A. van Rees, R. M. Oldenbeuving, J. P. Epping, R. G. Heideman, K. Worhoff, A. Leinse, D. Geuzebroek, E. Schreuder, P. W. L. van Dijk, I. Visscher, C. Taddei, Y. Fan, C. Taballione, Y. Liu, D. Marpaung, L. Zhuang, M. Benelajla, and K.-J. Boller, "Low-loss $\mathrm{Si}_{3} \mathrm{~N}_{4}$ TriPleX optical waveguides: technology and applications overview,” IEEE J. Sel. Top. Quantum Electron. 24, 4400321 (2018).

115. B. J. Eggleton, C. G. Poulton, and R. Pant, "Inducing and harnessing stimulated Brillouin scattering in photonic integrated circuits," Adv. Opt. Photon. 5, 536-587 (2013).

116. A. Choudhary, Y. Liu, D. Marpaung, and B. J. Eggleton, "Brillouin filtering with enhanced noise performance and linearity using anti-Stokes interactions," in Conference on Lasers and Electro-Optics (OSA, 2018), paper STu4F.2.

117. A. Mahendra, Y. Liu, E. Magi, A. Choudhary, D. Marpaung, and B. J. Eggleton, "High link performance of Brillouin-loss based microwave bandpass photonic filters," OSA Continuum 1, 1287-1297 (2018).

118. Y. Xie, Z. Geng, L. Zhuang, M. Burla, C. Taddei, M. Hoekman, A. Leinse, C. G. H. Roeloffzen, K.-J. Boller, and A. J. Lowery, "Programmable optical processor chips: toward photonic RF filters with DSP-level flexibility and MHz-band selectivity," Nanophotonics 7, 421-454 (2017).

119. C. Roeloffzen, L. Zhuang, R. Heideman, A. Borreman, and W. V. Etten, "Ring resonator-based tunable optical delay line in LPCVD waveguide technology," in Proceedings of the annual Symposium of the IEEE/LEOS Benelux Chapter, December 1-2 (2005), Vol. 1, pp. 79-82.

120. D. Rabus, Integrated Ring Resonators, Vol. 127 of Springer Series in Optical Sciences (Springer, 2007).

121. W. Bogaerts, P. De Heyn, T. Van Vaerenbergh, K. De Vos, S. Kumar Selvaraja, T. Claes, P. Dumon, P. Bienstman, D. Van Thourhout, and R. Baets, "Silicon microring resonators," Laser Photon. Rev. 6, 47-73 (2012).

122. A. Yariv, "Universal relations for coupling of optical power between microresonators and dielectric waveguides," Electron. Lett. 36, 321-322 (2000).

123. P. R. Prucnal, B. J. Shastri, and M. C. Teich, Neuromorphic Photonics (CRC Press/Taylor \& Francis, 2017), Vol. 1.

124. G. Li, J. Yao, H. Thacker, A. Mekis, X. Zheng, I. Shubin, Y. Luo, J.-H. Lee, K. Raj, J. E. Cunningham, and A. V. Krishnamoorthy, "Ultralow-loss, high-density 
SOI optical waveguide routing for macrochip interconnects," Opt. Express 20, 12035-12039 (2012).

125. S. A. Miller, M. Yu, X. Ji, A. G. Griffith, J. Cardenas, A. L. Gaeta, and M. Lipson, "Low-loss silicon platform for broadband mid-infrared photonics," Optica 4, 707-712 (2017).

126. M. Á. Guillén-Torres, K. Murray, H. Yun, M. Caverley, E. Cretu, L. Chrostowski, and N. A. F. Jaeger, "Effects of backscattering in high-Q, largearea silicon-on-insulator ring resonators," Opt. Lett. 41, 1538-1541 (2016).

127. T. Pett, J. H. Lee, Y. Ehrlichman, H. Gevorgyan, A. Khilo, and M. Popovic, "Photonics-based microwave radiometer for hyperspectral Earth remote sensing," in International Topical Meeting on Microwave Photonics (MWP) (IEEE, 2018), pp. 1-4.

128. D. J. Blumenthal, R. Heideman, D. Geuzebroek, A. Leinse, and C. Roeloffze, "Silicon nitride in silicon photonics," in Proceedings of the IEEE Special Issue on Silicon Photonics (Invited) (2018).

129. J. B. Khurgin and P. A. Morton, "Tunable wideband optical delay line based on balanced coupled resonator structures," Opt. Lett. 34, 2655-2657 (2009).

130. J. Cardenas, M. A. Foster, N. Sherwood-Droz, C. B. Poitras, H. L. R. Lira, B. Zhang, A. L. Gaeta, J. B. Khurgin, P. Morton, and M. Lipson, "Wide-bandwidth continuously tunable optical delay line using silicon microring resonators," Opt. Express 18, 26525-26534 (2010).

131. L. Zhuang, M. Hoekman, W. Beeker, A. Leinse, R. Heideman, P. van Dijk, and C. Roeloffzen, "Novel low-loss waveguide delay lines using Vernier ring resonators for on-chip multi- $\lambda$ microwave photonic signal processors," Laser Photon. Rev. 7, 994-1002 (2013).

132. L. Zhuang, M. Burla, C. Taddei, C. G. H. Roeloffzen, M. Hoekman, A. Leinse, K. J. Boller, and A. J. Lowery, "Integrated microwave photonic splitter with reconfigurable amplitude, phase, and delay offsets," Opt. Lett. 40, 5618-5621 (2015).

133. F. Xia, L. Sekaric, and Y. Vlasov, "Ultracompact optical buffers on a silicon chip," Nat. Photonics 1, 65-71 (2007).

134. F. Xia, M. Rooks, L. Sekaric, and Y. Vlasov, "Ultra-compact high order ring resonator filters using submicron silicon photonic wires for on-chip optical interconnects," Opt. Express 15, 11934-11941 (2007).

135. A. Melloni, A. Canciamilla, C. Ferrari, F. Morichetti, L. O'Faolain, T. F. Krauss, R. De La Rue, A. Samarelli, and M. Sorel, "Tunable delay lines in silicon photonics: coupled resonators and photonic crystals, a comparison," IEEE Photon. J. 2, 181-194 (2010).

136. R. Orta, P. Savi, R. Tascone, and D. Trinchero, "Synthesis of multiple-ringresonator filters for optical systems," IEEE Photon. Technol. Lett. 7, 1447-1449 (1995).

137. P. Dong, N.-N. Feng, D. Feng, W. Qian, H. Liang, D. C. Lee, B. J. Luff, T. Banwell, A. Agarwal, P. Toliver, R. Menendez, T. K. Woodward, and M. Asghari, "GHz-bandwidth optical filters based on high-order silicon ring resonators," Opt. Express 18, 23784-23789 (2010).

138. T. A. Huffman, G. M. Brodnik, C. Pinho, S. Gundavarapu, D. Baney, and D. J. Blumenthal, "Integrated resonators in an ultralow loss $\mathrm{Si}_{3} \mathrm{~N}_{4} / \mathrm{SiO}_{2}$ platform for multifunction applications," IEEE J. Sel. Top. Quantum Electron. 24, 5900209 (2018).

139. A. Melloni, "Synthesis of a parallel-coupled ring-resonator filter," Opt. Lett. 26, 917-919 (2001).

140. R. Grover, V. Van, T. A. Ibrahim, P. P. Absil, L. C. Calhoun, F. G. Johnson, J. V. Hryniewicz, and P. T. Ho, "Parallel-cascaded semiconductor microring 
resonators for high-order and wide-FSR filters," J. Lightwave Technol. 20, 900-905 (2002).

141. R. A. Cohen, O. Amrani, and S. Ruschin, "Linearized electro-optic silicon racetrack modulator based on double injection method," in International Topical Meeting on Microwave Photonics, MWP 2015-Conference Proceedings (2015), Vol. 23, pp. 5578-5587.

142. R. A. Cohen, O. Amrani, and S. Ruschin, "Response shaping with a silicon ring resonator via double injection," Nat. Photonics 12, 706-712 (2018).

143. L. Xu, Y. Yu, X. Liu, X. Shu, and X. Zhang, "An optical all-pass filter realized by using self-compensation of loss," arxiv: 1905.10020 (2019).

144. W. Zhang and J. Yao, "A fully reconfigurable waveguide Bragg grating for programmable photonic signal processing," Nat. Commun. 9, 1396 (2018).

145. S. Khan and S. Fathpour, "Demonstration of complementary apodized cascaded grating waveguides for tunable optical delay lines," Opt. Lett. 38, 3914-3917 (2013).

146. W. Zhang and J. Yao, "Electrically programmable on-chip equivalent-phaseshifted waveguide Bragg grating on silicon,” J. Lightwave Technol. 37, 314-322 (2019).

147. X. Han and J. Yao, "Bandstop-to-bandpass microwave photonic filter using a phase-shifted fiber Bragg grating," J. Lightwave Technol. 33, 5133-5139 (2015).

148. M. Burla, L. R. Cortés, M. Li, X. Wang, L. Chrostowski, and J. Azaña, "Integrated waveguide Bragg gratings for microwave photonics signal processing," Opt. Express 21, 25120-25147 (2013).

149. D. Huang, M. A. Tran, J. Guo, J. Peters, T. Komljenovic, A. Malik, P. A. Morton, and J. E. Bowers, "High-power sub-kHz linewidth lasers fully integrated on silicon," Optica 6, 745-752 (2019).

150. E. S. Hosseini, J. D. B. B. Purnawirman, J. Sun, G. Leake, T. N. Adam, D. D. Coolbaugh, and M. R. Watts, "CMOS-compatible $75 \mathrm{~mW}$ erbium-doped distributed feedback laser," Opt. Lett. 39, 3106-3109 (2014).

151. M. Belt and D. J. Blumenthal, "Erbium-doped waveguide DBR and DFB laser arrays integrated within an ultra-low-loss $\mathrm{Si}_{3} \mathrm{~N}_{4}$ platform," Opt. Express 22, 10655-10660 (2014).

152. K. Oda, N. Takato, H. Toba, and K. Nosu, "A wide-band guided-wave periodic multi/demultiplexer with a ring resonator for optical FDM transmission systems," J. Lightwave Technol. 6, 1016-1023 (1988).

153. L. Zhuang, M. Hoekman, R. M. Oldenbeuving, K.-J. Boller, and C. G. H. Roeloffzen, "CRIT-alternative narrow-passband waveguide filter for microwave photonic signal processors," IEEE Photon. Technol. Lett. 26, 1034-1037 (2014).

154. L. Zhuang, C. Zhu, B. Corcoran, M. Burla, C. G. H. Roeloffzen, A. Leinse, J. Schröder, and A. J. Lowery, "Sub-GHz-resolution C-band Nyquist-filtering interleaver on a high-index-contrast photonic integrated circuit," Opt. Express 24, 5715-5727 (2016).

155. L. Zhuang, C. Zhu, Y. Xie, M. Burla, C. G. H. Roeloffzen, M. Hoekman, B. Corcoran, and A. J. Lowery, "Nyquist-filtering (de)multiplexer using a ring resonator assisted interferometer circuit," J. Lightwave Technol. 34, 1732-1738 (2016).

156. L. Zhuang, W. Beeker, A. Leinse, R. Heideman, P. van Dijk, and C. G. Roeloffzen, "Novel wideband microwave polarization network using a fully-reconfigurable photonic waveguide interleaver with a two-ring resonator-assisted asymmetric Mach-Zehnder," Opt. Express 21, 3114-3124 (2013). 
157. Z. Geng, Y. Xie, L. Zhuang, M. Burla, M. Hoekman, C. G. H. Roeloffzen, and A. J. Lowery, "Photonic integrated circuit implementation of a sub-GHz-selectivity frequency comb filter for optical clock multiplication," Opt. Express 25, 2763527645 (2017).

158. B. J. Eggleton, C. G. Poulton, P. T. Rakich, M. J. Steel, and G. Bahl, "Brillouin integrated photonics," Nat. Photonics 13, 664-677 (2019).

159. L. Razzari, D. Duchesne, M. Ferrera, R. Morandotti, S. Chu, B. E. Little, and D. J. Moss, "CMOS-compatible integrated optical hyper-parametric oscillator," Nat. Photonics 4, 41-45 (2010).

160. J. S. Levy, A. Gondarenko, M. A. Foster, A. C. Turner-Foster, A. L. Gaeta, and M. Lipson, "CMOS-compatible multiple-wavelength oscillator for on-chip optical interconnects," Nat. Photonics 4, 37-40 (2010).

161. A. Pasquazi, M. Peccianti, L. Razzari, D. J. Moss, S. Coen, M. Erkintalo, Y. K. Chembo, T. Hansson, S. Wabnitz, P. Del'Haye, X. Xue, A. M. Weiner, and R. Morandotti, "Micro-combs: a novel generation of optical sources," Phys. Rep. 729, 1-81 (2018).

162. R. Pant, C. G. Poulton, D.-Y. Choi, H. McFarlane, S. Hile, E. Li, L. Thevenaz, B. Luther-Davies, S. J. Madden, and B. J. Eggleton, "On-chip stimulated Brillouin scattering," Opt. Express 19, 8285-8290 (2011).

163. C. Wolff, M. J. Steel, B. J. Eggleton, and C. G. Poulton, "Stimulated Brillouin scattering in integrated photonic waveguides: forces, scattering mechanisms, and coupled-mode analysis," Phys. Rev. A 92, 013836 (2015).

164. C. G. Poulton, R. Pant, and B. J. Eggleton, "Acoustic confinement and stimulated Brillouin scattering in integrated optical waveguides," J. Opt. Soc. Am. B 30, 2657-2664 (2013).

165. D. Marpaung, M. Pagani, B. Morrison, and B. J. Eggleton, "Nonlinear integrated microwave photonics," J. Lightwave Technol. 32, 3421-3427 (2014).

166. M. Merklein, A. Casas-Bedoya, D. Marpaung, T. F. S. Buttner, M. Pagani, B. Morrison, I. V. Kabakova, and B. J. Eggleton, "Stimulated Brillouin scattering in photonic integrated circuits: novel applications and devices," IEEE J. Sel. Top. Quantum Electron. 22, 336-346 (2016).

167. A. Choudhary, Y. Liu, D. Marpaung, and B. J. Eggleton, "On-chip Brillouin filtering of RF and optical signals," IEEE J. Sel. Top. Quantum Electron. 24, 7600211 (2018).

168. Y. Liu, A. Choudhary, D. Marpaung, and B. J. Eggleton, "Chip-based Brillouin processing for phase control of RF signals," IEEE J. Quantum Electron. 54, 1-13 (2018).

169. M. Merklein, B. Stiller, and B. J. Eggleton, "Brillouin-based light storage and delay techniques," J. Opt. 20, 083003 (2018).

170. A. Loayssa and F. J. Lahoz, "Broad-band RF photonic phase shifter based on stimulated Brillouin scattering and single-sideband modulation," IEEE Photon. Technol. Lett. 18, 208-210 (2006).

171. Y. Okawachi, M. S. Bigelow, J. E. Sharping, Z. Zhu, A. Schweinsberg, D. J. Gauthier, R. W. Boyd, and A. L. Gaeta, "Tunable all-optical delays via Brillouin slow light in an optical fiber," Phys. Rev. Lett. 94, 153902 (2005).

172. W. Li, N. H. Zhu, L. X. Wang, J. S. Wang, J. G. Liu, Y. Liu, X. Q. Qi, L. Xie, W. Chen, X. Wang, and W. Han, "True-time delay line with separate carrier tuning using dual-parallel MZM and stimulated Brillouin scattering-induced slow light," Opt. Express 19, 12312-12324 (2011).

173. J. Sancho, S. Chin, M. Sagues, A. Loayssa, J. Lloret, I. Gasulla, S. Sales, L. Thevenaz, and J. Capmany, "True time delay on tunable microwave photonic filter based on stimulated Brillouin scattering in fibers," in 36th European 
Conference and Exhibition on Optical Communication (IEEE, 2010), Vol. 22, pp. 1-3.

174. T. Schneider, K. Jamshidi, and S. Preubler, "Quasi-light storage: a method for the tunable storage of optical packets with a potential delay-bandwidth product of several thousand bits," J. Lightwave Technol. 28, 2586-2592 (2010).

175. T. Schneider and S. Preußler, "Quasi-light storage for optical data packets," J. Vis. Exp. 84, e50468 (2014).

176. H. Peng, T. Sun, C. Zhang, X. Xie, P. Guo, L. Zhu, W. Hu, and Z. Chen, "Tunable DC-60 GHz RF generation based on a dual loop Brillouin optoelectronic oscillator," in European Conference on Optical Communication (ECOC) (2014).

177. X. S. Yao, "High-quality microwave signal generation by use of Brillouin scattering in optical fibers," Opt. Lett. 22, 1329-1331 (1997).

178. E. Ippen and R. Stolen, "Stimulated Brillouin scattering in optical fibers," Appl. Phys. Lett. 21, 539-541 (1972).

179. A. Kobyakov, M. Sauer, and D. Chowdhury, "Stimulated Brillouin scattering in optical fibers," Adv. Opt. Photon. 2, 1-59 (2010).

180. W. Zhang and R. A. Minasian, "Ultrawide tunable microwave photonic notch filter based on stimulated Brillouin scattering," IEEE Photon. Technol. Lett. 24, 1182-1184 (2012).

181. I. Aryanfar, A. Choudhary, S. Shahnia, M. Pagani, Y. Liu, D. Marpaung, and B. J. Eggleton, "Signal interference RF photonic bandstop filter," Opt. Express 24, 14995-15004 (2016).

182. A. Choudhary, Y. Liu, B. Morrison, K. Vu, D.-Y. Choi, P. Ma, S. Madden, D. Marpaung, and B. J. Eggleton, "High-resolution, on-chip RF photonic signal processor using Brillouin gain shaping and RF interference," Sci. Rep. 7, 5932 (2017).

183. A. Choudhary, B. Morrison, I. Aryanfar, S. Shahnia, M. Pagani, Y. Liu, K. Vu, S. Madden, D. Marpaung, and B. J. Eggleton, "Advanced integrated microwave signal processing with giant on-chip Brillouin gain," J. Lightwave Technol. 35, 846-854 (2017).

184. I. Aryanfar, D. Marpaung, A. Choudhary, Y. Liu, K. Vu, D.-Y. Choi, P. Ma, S. Madden, and B. J. Eggleton, "Chip-based Brillouin radio frequency photonic phase shifter and wideband time delay," Opt. Lett. 42, 1313-1316 (2017).

185. A. Choudhary, M. Pelusi, D. Marpaung, T. Inoue, K. Vu, P. Ma, D.-Y. Choi, S. Madden, S. Namiki, and B. J. Eggleton, "On-chip Brillouin purification for frequency comb-based coherent optical communications," Opt. Lett. 42, 5074-5077 (2017).

186. E. Giacoumidis, A. Choudhary, E. Magi, D. Marpaung, K. Vu, P. Ma, D.-Y. Choi, S. Madden, B. Corcoran, M. Pelusi, and B. J. Eggleton, "Chipbased Brillouin processing for carrier recovery in self-coherent optical communications," Optica 5, 1191-1199 (2018).

187. S. Preussler and T. Schneider, "Stimulated Brillouin scattering gain bandwidth reduction and applications in microwave photonics and optical signal processing," Opt. Eng. 55, 031110 (2015).

188. H. Shin, J. A. Cox, R. Jarecki, A. Starbuck, Z. Wang, and P. T. Rakich, "Control of coherent information via on-chip photonic-phononic emitter-receivers," Nat. Commun. 6, 6427 (2015).

189. B. Morrison, A. Casas-Bedoya, G. Ren, K. Vu, Y. Liu, A. Zarifi, T. G. Nguyen, D.-Y. Choi, D. Marpaung, S. J. Madden, A. Mitchell, and B. J. Eggleton, "Compact Brillouin devices through hybrid integration on silicon," Optica 4, 847-854 (2017). 
190. R. Califa, D. Munk, H. Genish, Y. Kaganovskii, I. Bakish, M. Rosenbluh, and A. Zadok, "Large one-time photo-induced tuning of directional couplers in chalcogenide-on-silicon platform," Opt. Express 23, 28234-28243 (2015).

191. B. Shen, H. Lin, F. Merget, S. S. Azadeh, C. Li, G.-Q. Lo, K. A. Richardson, J. $\mathrm{Hu}$, and J. Witzens, "Broadband couplers for hybrid silicon-chalcogenide glass photonic integrated circuits," Opt. Express 27, 13781-13792 (2019).

192. J. Wu, X. Xu, T. G. Nguyen, S. T. Chu, B. E. Little, R. Morandotti, A. Mitchell, and D. J. Moss, "A highly versatile microwave photonic filter based on an integrated optical frequency comb source," in Conference on Lasers and Electro-Optics (CLEO) (2018).

193. D. J. Moss, R. Morandotti, A. L. Gaeta, and M. Lipson, "New CMOS-compatible platforms based on silicon nitride and Hydex for nonlinear optics," Nat. Photonics 7, 597-607 (2013).

194. J. Li, Y. Dai, F. Yin, W. Li, M. Li, H. Chen, and K. Xu, "Megahertz-resolution programmable microwave shaper," Opt. Lett. 43, 1878-1881 (2018).

195. J. Hu, J. He, J. Liu, A. S. Raja, M. Karpov, A. Lukashchuk, T. J. Kippenberg, and C.-S. Brès, "Reconfigurable radiofrequency filters based on versatile soliton microcombs," arXiv:2001.11538 (2020).

196. C. Wang, M. Zhang, X. Chen, M. Bertrand, A. Shams-Ansari, S. Chandrasekhar, P. Winzer, and M. Lončar, "Integrated lithium niobate electro-optic modulators operating at CMOS-compatible voltages," Nature 562, 101-104 (2018).

197. C. Wang, M. Zhang, M. Yu, R. Zhu, H. Hu, and M. Loncar, "Monolithic lithium niobate photonic circuits for Kerr frequency comb generation and modulation," Nat. Commun. 10, 978 (2019).

198. C. Wang, M. Zhang, B. Stern, M. Lipson, and M. Lončar, "Nanophotonic lithium niobate electro-optic modulators," Opt. Express 26, 1547-1555 (2018).

199. M. Zhang, B. Buscaino, C. Wang, A. Shams-Ansari, C. Reimer, R. Zhu, J. M. Kahn, and M. Lončar, "Broadband electro-optic frequency comb generation in a lithium niobate microring resonator," Nature 568, 373-377 (2019).

200. M. Zhang, C. Wang, R. Cheng, A. Shams-Ansari, and M. Lončar, "Monolithic ultra-high- $Q$ lithium niobate microring resonator," Optica 4, 1536-1537 (2017).

201. C. Wang, M. J. Burek, Z. Lin, H. A. Atikian, V. Venkataraman, I.-C. Huang, P. Stark, and M. Lončar, "Integrated high quality factor lithium niobate microdisk resonators," Opt. Express 22, 30924-30933 (2014).

202. M. Smit, X. Leijtens, H. Ambrosius, E. Bente, J. van der Tol, B. Smalbrugge, T. de Vries, E.-J. Geluk, J. Bolk, R. van Veldhoven, L. Augustin, P. Thijs, D. D’Agostino, H. Rabbani, K. Lawniczuk, S. Stopinski, S. Tahvili, A. Corradi, E. Kleijn, D. Dzibrou, M. Felicetti, E. Bitincka, V. Moskalenko, J. Zhao, R. Santos, G. Gilardi, W. Yao, K. Williams, P. Stabile, P. Kuindersma, J. Pello, S. Bhat, Y. Jiao, D. Heiss, G. Roelkens, M. Wale, P. Firth, F. Soares, N. Grote, M. Schell, H. Debregeas, M. Achouche, J.-L. Gentner, A. Bakker, T. Korthorst, D. Gallagher, A. Dabbs, A. Melloni, F. Morichetti, D. Melati, A. Wonfor, R. Penty, R. Broeke, B. Musk, and D. Robbins, "An introduction to InP-based generic integration technology," Semicond. Sci. Technol. 29, 083001 (2014).

203. H. Zhao, S. Pinna, B. Song, L. Megalini, S. S. Brunelli, L. Coldren, and J. Klamkin, "High-power integrated indium phosphide transmitter for free space optical communications," in Conference on Lasers and Electro-Optics (OSA, 2018), Vol. 2, paper JW2A.52.

204. F. A. Kish, D. Welch, R. Nagarajan, J. L. Pleumeekers, V. Lal, M. Ziari, A. Nilsson, M. Kato, S. Murthy, P. Evans, S. W. Corzine, M. Mitchell, P. Samra, M. Missey, S. DeMars, R. P. Schneider, M. S. Reffle, T. Butrie, J. T. Rahn, M. Van Leeuwen, J. W. Stewart, D. J. Lambert, R. C. Muthiah, H. S. Tsai, J. S. Bostak, A. Dentai, K. T. Wu, H. Sun, D. J. Pavinski, J. Zhang, J. Tang, J. McNicol, M. 
Kuntz, V. Dominic, B. D. Taylor, R. A. Salvatore, M. Fisher, A. Spannagel, E. Strzelecka, P. Studenkov, M. Raburn, W. Williams, D. Christini, K. J. Thomson, S. S. Agashe, R. Malendevich, G. Goldfarb, S. Melle, C. Joyner, M. Kaufman, and S. G. Grubb, "Current status of large-scale InP photonic integrated circuits," IEEE J. Sel. Top. Quantum Electron. 17, 1470-1489 (2011).

205. Y. A. Akulova, G. A. Fish, P. C. Koh, C. L. Schow, P. Kozodoy, A. P. Dahl, S. Nakagawa, M. C. Larson, M. P. Mack, T. A. Strand, C. W. Coldren, E. Hegblom, S. K. Penniman, T. Wipiejewski, and L. A. Coldren, "Widely tunable electroabsorption-modulated sampled-grating DBR laser transmitter," IEEE J. Sel. Top. Quantum Electron. 8, 1349-1357 (2002).

206. J. D. Merlier, K. Mizutani, S. Sudo, K. Naniwae, Y. Furushima, S. Sato, K. Sato, K. Kudo, and S. Member, "Full C-band external cavity wavelength tunable laser using a liquid-crystal-based tunable mirror," IEEE Photon. Technol. Lett. 17, 681-683 (2005).

207. G. Letal, K. Prosyk, R. Millett, D. Macquistan, S. Paquet, O. Thibault-Maheu, J.F. Gagné, P.-L. Fortin, R. Dowlatshahi, B. Rioux, T. S. Thorpe, M. Hisko, R. Ma, and I. Woods, "Low loss InP C-band IQ modulator with 40GHz bandwidth and $1.5 \mathrm{~V}$ V $\pi$," in Optical Fiber Communication Conference (2015), paper Th4E.3.

208. S. Lange, S. Wolf, J. Lutz, L. Altenhain, R. Schmid, R. Kaiser, M. Schell, C. Koos, and S. Randel, "100 GBd intensity modulation and direct detection with an InP-based monolithic DFB laser Mach-Zehnder modulator," J. Lightwave Technol. 36, 97-102 (2018).

209. R. A. Griffin, S. K. Jones, N. Whitbread, S. C. Heck, and L. N. Langley, "InP Mach-Zehnder modulator platform for 10/40/100/200-Gb/s operation,” IEEE J. Sel. Top. Quantum Electron. 19, 158-166 (2013).

210. L. R. Chen, "Silicon photonics for microwave photonics applications," J. Lightwave Technol. 35, 824-835 (2017).

211. L. R. Chen, P. Moslemi, Z. Wang, M. Ma, and R. Adams, "Integrated microwave photonics for spectral analysis, waveform generation, and filtering," IEEE Photon. Technol. Lett. 30, 1838-1841 (2018).

212. A. Biberman, M. J. Shaw, E. Timurdogan, J. B. Wright, and M. R. Watts, "Ultralow-loss silicon ring resonators," Opt. Lett. 37, 4236-4268 (2012).

213. P. Dong, L. Chen, and Y.-K. Chen, "High-speed low-voltage single-drive pushpull silicon Mach-Zehnder modulators," Opt. Express 20, 6163-6169 (2012).

214. T. Baba, S. Akiyama, M. Imai, N. Hirayama, H. Takahashi, Y. Noguchi, T. Horikawa, and T. Usuki, “50-Gb/s ring-resonator-based silicon modulator," Opt. Express 21, 11869-11876 (2013).

215. X. Tu, T.-Y. Liow, J. Song, X. Luo, Q. Fang, M. Yu, and G.-Q. Lo, “50-Gb/s silicon optical modulator with traveling-wave electrodes," Opt. Express 21, 12776$12782(2013)$.

216. D. J. Thomson, F. Y. Gardes, J. M. Fedeli, S. Zlatanovic, Y. Hu, B. P. P. Kuo, E. Myslivets, N. Alic, S. Radic, G. Z. Mashanovich, and G. T. Reed, "50-Gb/s silicon optical modulator," IEEE Photon. Technol. Lett. 24, 234-236 (2012).

217. L. Vivien, A. Polzer, D. Marris-Morini, J. Osmond, J. M. Hartmann, P. Crozat, E. Cassan, C. Kopp, H. Zimmermann, and J. M. Fédéli, "Zero-bias 40 Gbit/s germanium waveguide photodetector on silicon," Opt. Express 20, 1096-1101 (2012).

218. T. Yin, R. Cohen, M. M. Morse, G. Sarid, Y. Chetrit, D. Rubin, and M. J. Paniccia, "31 GHz Ge $n$-i-p waveguide photodetectors on silicon-on-insulator substrate," Opt. Express 15, 13965-13971 (2007).

219. L. Vivien, J. Osmond, J.-M. Fédéli, D. Marris-Morini, P. Crozat, J.-F. Damlencourt, E. Cassan, Y. Lecunff, and S. Laval, "42 GHz pin germanium 
photodetector integrated in a silicon-on-insulator waveguide," Opt. Express 17, 6252-6257 (2009).

220. S. Lischke, D. Knoll, C. Mai, L. Zimmermann, A. Peczek, M. Kroh, A. Trusch, E. Krune, K. Voigt, and A. Mai, "High bandwidth, high responsivity waveguidecoupled germanium p-i-n photodiode," Opt. Express 23, 27213-27220 (2015).

221. W. Zhang and J. Yao, "On-chip silicon photonic integrated frequency-tunable bandpass microwave photonic filter," Opt. Lett. 43, 3622-3625 (2018).

222. M. J. R. Heck, J. F. Bauters, M. L. Davenport, J. K. Doylend, S. Jain, G. Kurczveil, S. Srinivasan, and J. E. Bowers, "Hybrid silicon photonic integrated circuit technology,” J. Sel. Top. Quantum Electron. 19, 6100117 (2013).

223. G.-H. Duan, C. Jany, A. Le Liepvre, A. Accard, M. Lamponi, D. Make, P. Kaspar, G. Levaufre, N. Girard, F. Lelarge, J.-M. Fedeli, A. Descos, B. Ben Bakir, S. Messaoudene, D. Bordel, S. Menezo, G. de Valicourt, S. Keyvaninia, G. Roelkens, D. Van Thourhout, D. J. Thomson, F. Y. Gardes, and G. T. Reed, "Hybrid III-V on silicon lasers for photonic integrated circuits on silicon," IEEE J. Sel. Top. Quantum Electron. 20, 158-170 (2014).

224. T. Komljenovic, M. Davenport, J. Hulme, A. Liu, C. Santis, A. Spott, S. Srinivasan, E. Stanton, C. Zhang, and J. Bowers, "Heterogeneous silicon photonic integrated circuits," J. Lightwave Technol. 34, 20-35 (2015).

225. N. Kobayashi, K. Sato, M. Namiwaka, K. Yamamoto, S. Watanabe, T. Kita, H. Yamada, and H. Yamazaki, "Silicon photonic hybrid ring-filter external cavity wavelength tunable lasers," J. Lightwave Technol. 33, 1241-1246 (2015).

226. Z. Wang, A. Abbasi, U. Dave, A. De Groote, S. Kumari, B. Kunert, C. Merckling, M. Pantouvaki, Y. Shi, B. Tian, K. Van Gasse, J. Verbist, R. Wang, W. Xie, J. Zhang, Y. Zhu, J. Bauwelinck, X. Yin, Z. Hens, J. Van Campenhout, B. Kuyken, R. Baets, G. Morthier, D. Van Thourhout, and G. Roelkens, "Novel light source integration approaches for silicon photonics," Laser Photon. Rev. 11, 1-21 (2017).

227. C. T. Santis, S. T. Steger, Y. Vilenchik, A. Vasilyev, and A. Yariv, "Highcoherence semiconductor lasers based on integral high- $Q$ resonators in hybrid Si/III-V platforms," Proc. Natl. Acad. Sci. USA 111, 2879-2884 (2014).

228. T. Komljenovic, S. Srinivasan, E. Norberg, M. Davenport, G. Fish, and J. E. Bowers, "Widely tunable narrow-linewidth monolithically integrated externalcavity semiconductor lasers," IEEE J. Sel. Top. Quantum Electron. 21, 1501909 (2015).

229. C. Xiang, W. Jin, J. Guo, J. D. Peters, M. J. Kennedy, J. Selvidge, P. A. Morton, and J. E. Bowers, "Narrow-linewidth III- $/ / \mathrm{Si} / \mathrm{Si}_{3} \mathrm{~N}_{4}$ laser using multilayer heterogeneous integration," Optica 7, 20-21 (2020).

230. J. C. Hulme, M. J. Kennedy, R.-L. Chao, L. Liang, T. Komljenovic, J.-W. Shi, B. Szafraniec, D. Baney, and J. E. Bowers, "Fully integrated microwave frequency synthesizer on heterogeneous silicon-III/V," Opt. Express 25, 2422-2431 (2017).

231. A. Ribeiro, A. Ruocco, L. Vanacker, and W. Bogaerts, "Demonstration of a $4 \times 4$-port self-configuring universal linear optical component," in Progress In Electromagnetics Research Symposium (PIERS) (2016), Vol. 3, pp. 3372-3375.

232. W. R. Clements, P. C. Humphreys, B. J. Metcalf, W. S. Kolthammer, and I. A. Walsmley, "Optimal design for universal multiport interferometers," Optica 3, 1460-1465 (2016).

233. D. A. B. Miller, "Perfect optics with imperfect components," Optica 2, 747-750 (2015).

234. D. Pérez, I. Gasulla, and J. Capmany, "Software-defined reconfigurable microwave photonics processor," Opt. Express 23, 14640-14654 (2015). 
235. D. Pérez, I. Gasulla, J. Capmany, and R. A. Soref, "Reconfigurable lattice mesh designs for programmable photonic processors," Opt. Express 24, 12093-12106 (2016).

236. D. Pérez and J. Capmany, "Scalable analysis for arbitrary photonic integrated waveguide meshes," Optica 6, 19-27 (2019).

237. X. Zou, F. Zou, Z. Cao, B. Lu, X. Yan, G. Yu, X. Deng, B. Luo, L. Yan, W. Pan, J. Yao, and A. M. J. Koonen, "A multifunctional photonic integrated circuit for diverse microwave signal generation, transmission, and processing," Laser Photon. Rev. 13, 1800240 (2019).

238. G. P. Agrawal, Fiber-Optic Communication Systems (Wiley, 2012), Vol. 222.

239. D. A. I. Marpaung, "High dynamic range analog photonic links: design and implementation," Ph.D. thesis (University of Twente, 2009).

240. J. D. McKinney, M. Godinez, V. J. Urick, S. Thaniyavarn, W. Charczenko, and K. J. Williams, "Sub-10-dB noise figure in a multiple-GHz analog optical link," IEEE Photon. Technol. Lett. 19, 465-467 (2007).

241. A. Karim and J. Devenport, "Low noise figure microwave photonic link," in IEEE/MTT-S International Microwave Symposium (2007), pp. 1519-1522.

242. A. Karim and J. Devenport, "Noise figure reduction in externally modulated analog fiber-optic links," IEEE Photon. Technol. Lett. 19, 312-314 (2007).

243. H. V. Roussell, M. D. Regan, J. L. Prince, C. H. Cox, J. X. Chen, W. K. Burns, G. E. Betts, E. I. Ackerman, and J. C. Campbell, "Gain, noise figure and bandwidthlimited dynamic range of a low-biased external modulation link," in $M W P$ 2007-2007 IEEE International Topical Meeting on Microwave Photonics (2007), pp. 84-87.

244. A. Savchenkov, V. Ilchenko, E. Dale, D. Seidel, A. Matsko, and L. Maleki, "Agile high-Q RF photonic zooming filter," IEEE Photon. Technol. Lett. 28, 43-46 (2016).

245. Y. Liu, D. Marpaung, A. Choudhary, and B. J. Eggleton, "Lossless and highresolution RF photonic filter," in IEEE Avionics and Vehicle Fiber-Optics and Photonics Conference (AVFOP) (IEEE, 2016), Vol. 41, pp. 185-186.

246. V. J. Urick, M. E. Godinez, P. S. Devgan, J. D. McKinney, and F. Bucholtz, "Analysis of an analog fiber-optic link employing a low-biased Mach-Zehnder modulator followed by an erbium-doped fiber amplifier," J. Lightwave Technol. 27, 2013-2019 (2009).

247. V. J. Urick, J. D. McKinney, J. F. Diehl, and K. J. Williams, "Fiber-optic links with all-photonic RF gain and low RF noise figure," in IEEE MTT-S International Microwave Symposium (IEEE, 2011), pp. 1-4.

248. L. Liu, T. Yang, and J.-J. Dong, "Microwave photonic filter with a continuously tunable central frequency using an SOI high- $Q$ microdisk resonator," Chin. Phys. B 23, 093201 (2014).

249. Y. Liu, J. Hotten, A. Choudhary, B. J. Eggleton, and D. Marpaung, "Lossless integrated RF photonic filter with record-low noise figure and $116 \mathrm{~dB}$ of dynamic range," in Conference on Lasers and Electro-Optics Pacific Rim (CLEO-PR) (IEEE, 2017), pp. 1-2.

250. Y. Liu, D. Marpaung, B. J. Eggleton, and A. Choudhary, "High-performance chip-assisted microwave photonic functionalities," IEEE Photon. Technol. Lett. 30, 1822-1825 (2018).

251. C. Feng, S. Preussler, and T. Schneider, "Sharp tunable and additional noise-free optical filter based on Brillouin losses," Photon. Res. 6, 132-137 (2018).

252. A. Mahendra, Y. Liu, E. Magi, A. Choudhary, D. Marpaung, and B. J. Eggleton, "High link performance of Brillouin-loss based microwave bandpass photonic filters," OSA Continuum 1, 1287 (2018). 
253. M. Garrett, Y. Liu, D.-Y. Choi, P. Ma, S. J. Madden, and B. J. Eggleton, "Highlink-gain and deep-rejection chip-based microwave photonic bandpass filter using moderate Brillouin gain," in Asia Communications and Photonics Conference (ACPC) (2019), Vol. 1, pp. 1-3.

254. Y. Fan, R. M. Oldenbeuving, C. G. Roeloffzen, M. Hoekman, D. Geskus, R. G. Heideman, and K.-J. Boller, " $290 \mathrm{~Hz}$ intrinsic linewidth from an integrated optical chip-based widely tunable InP- $\mathrm{Si}_{3} \mathrm{~N}_{4}$ hybrid laser," in Conference on Lasers and Electro-Optics (2017), 161, paper JTh5C.9.

255. P. A. Morton and M. Morton, "High-power, ultra-low noise hybrid lasers for microwave photonics and optical sensing," J. Lightwave Technol. 36, 5048-5057 (2018).

256. N. Li, Z. S. Purnawirman, E. Salih Magden, P. T. Callahan, K. Shtyrkova, M. Xin, A. Ruocco, C. Baiocco, E. P. Ippen, F. X. Kärtner, J. D. B. Bradley, D. Vermeulen, and M. R. Watts, "High-power thulium lasers on a silicon photonics platform," Opt. Lett. 42, 1181-1184 (2017).

257. J. H. Han, F. Boeuf, J. Fujikata, S. Takahashi, S. Takagi, and M. Takenaka, "Efficient low-loss InGaAsP/Si hybrid MOS optical modulator," Nat. Photonics 11, 486-490 (2017).

258. C. Wang, M. Zhang, X. Chen, M. Bertrand, A. Shams-Ansari, S. Chandrasekhar, P. Winzer, and M. Lončar, "Integrated lithium niobate electro-optic modulators operating at CMOS-compatible voltages," Nature 562, 101-104 (2018).

259. C. Zhang, P. A. Morton, J. B. Khurgin, J. D. Peters, and J. E. Bowers, "Ultralinear heterogeneously integrated ring-assisted Mach-Zehnder interferometer modulator on silicon," Optica 3, 1483-1488 (2016).

260. M. Burla, C. Hoessbacher, W. Heni, C. Haffner, Y. Fedoryshyn, D. Werner, T. Watanabe, H. Massler, D. L. Elder, L. R. Dalton, and J. Leuthold, "500 GHz plasmonic Mach-Zehnder modulator enabling sub-THz microwave photonics," APL Photon. 4, 056106 (2019).

261. M. J. Heck, J. F. Bauters, M. L. Davenport, D. T. Spencer, and J. E. Bowers, "Ultra-low loss waveguide platform and its integration with silicon photonics," Laser Photon. Rev. 8, 667-686 (2014).

262. J. F. Bauters, M. L. Davenport, M. J. R. Heck, J. K. Doylend, A. Chen, A. W. Fang, and J. E. Bowers, "Silicon on ultra-low-loss waveguide photonic integration platform," Opt. Express 21, 544-555 (2013).

263. X. Ji, F. A. S. Barbosa, S. P. Roberts, A. Dutt, J. Cardenas, Y. Okawachi, A. Bryant, A. L. Gaeta, and M. Lipson, "Ultra-low-loss on-chip resonators with sub-milliwatt parametric oscillation threshold," Optica 4, 619-624 (2017).

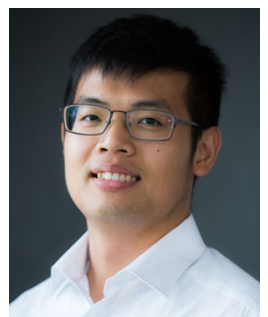

Yang Liu received a Bachelor's degree in optoelectronics from the Huazhong University of Science and Technology (HUST), Wuhan, China, in 2012 and a Master's degree in optical engineering from Wuhan National Laboratory for Optoelectronics (WNLO) in 2015. He received a Ph.D. degree in physics in the Nonlinear Photonics Group at the Centre for Ultrahigh Bandwidth Devices for Optical Systems (CUDOS), School of Physics, University of Sydney, Australia, in 2019. He is currently a Postdoctoral Research Associate leading integrated microwave photonics and hybrid integration research activities in The University of Sydney, Australia. Yang was the recipient of the Chinese Government Award for Distinguished Self-financed Student Abroad in 2019 and the MSCA IF fellowship in 2020. His research interests include the fundamentals and applications of nonlinear optics and microwave photonics in integrated photonics. He was the Vice President of OSA/SPIE student chapters 
at the University of Sydney from 2017 to 2018. He was a conference organization committee member for IONS KOALA 2018.

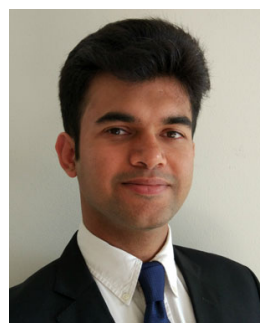

Amol Choudhary received a Bachelor of Engineering from the Delhi College of Engineering (now DTU), India; an Erasmus Mundus MSc in Photonics from the Gent University, Belgium, and the University of St. Andrews, UK; and a Ph.D. in Optoelectronics from the University of Southampton, UK. He worked as an EPSRC Doctoral Prize Fellow at the University of Southampton and as the Australian Government's Discovery Early Career Research Award (DECRA) fellow at the University of Sydney, Australia. He is currently a faculty member at the Department of Electrical Engineering at the Indian Institute of Technology (IIT) Delhi, India and heads the Ultra Fast Optical Communications and High-performance Integrated Photonics (UFO-CHIP) group. His research areas of interest are photon-phonon interactions, laser physics, integrated optics, nonlinear optics, ultrafast optics, optical communications, and microwave photonics. He is a senior member of the Optical Society (OSA). $\mathrm{He}$ is also the 2018 recipient of the OSA's Ivan P. Kaminow Outstanding Early Career Professional Prize and is a 2019 OSA Ambassador. He has more than 100 publications including 33 journal papers. He is a technical program committee member for CLEO USA 2017-20; SPIE Photonics Europe 2018, 2020; OSA Nonlinear Photonics 2020; and SPIE Optics and Photonics 2018-19.

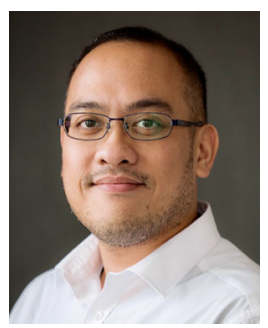

David Marpaung joined the University of Twente in January 2018 as an associate professor leading the Nonlinear Nanophotonics group. He received his Ph.D. degree in electrical engineering from the University of Twente, The Netherlands, in 2009. From 2009 to 2012, he was a postdoctoral researcher in the University of Twente, working on microwave photonic system integration for optical beamforming. He joined CUDOS University of Sydney, Australia, in August 2012 as a research fellow. David was the recipient of the 2015 Discovery Early Career Research Award (DECRA) from the Australian Research Council, the 2017 Vidi award, and the 2018 Start Up fellowship from the Netherlands Organisation for Scientific Research (NWO). His research interests include RF photonics, optomechanics, nonlinear optics, and phononics.

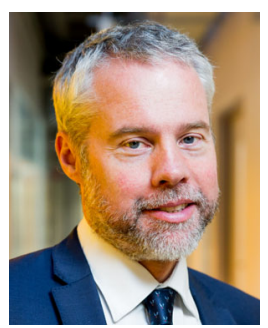

Benjamin J. Eggleton (F'10) received a Bachelor's (Hons.) degree in science and a Ph.D. degree in physics from the University of Sydney, Australia, in 1992 and 1996, respectively. In 1996, he joined Bell Laboratories, Lucent Technologies, Murray Hill, NJ, US, as a Postdoctoral Member of Staff, and then transferred to the Department of Optical Fiber Research. In 2000, he became the Research Director within the Specialty Fiber Business Division of Lucent Technologies. He is currently a professor of physics at the University of Sydney, the Director of the University of Sydney Nano Institute (Sydney Nano), and Co-Director of the NSW Smart Sensing Network (NSSN). He was previously the Director of the ARC Centre of 
Excellence for Ultrahigh Bandwidth Devices for Optical Systems (CUDOS). He is the author or coauthor of more than 500 journal publications. His research interests include nonlinear optics, all-optical signal processing, optical communications, photonic crystals, and stimulated Brillouin scattering. Dr. Eggleton is a Fellow of the Australian Academy of Science, the Australian Academy of Technology and Engineering (ATSE), The Optical Society, IEEE Photonics, and SPIE. He received the Pawsey Medal from the Australian Academy of Science, the 2004 Malcolm McIntosh Prize for Physical Scientist of the Year, the 2003 International Commission on Optics Prize, the 1998 Adolph Lomb Medal from The Optical Society (OSA), the Distinguished Lecturer Award from the IEEE/Lasers and Electro-Optics Society, and the Research and Development 100 Award. He was the President of the Australian Optical Society during 2008-2010, he served on the Board of Governor for IEEE Photonics from 2015-2017, and is currently an Editor-in-Chief of APL Photonics. 